\title{
Weaving the Quantum Optical Frequency Combs into Hypercubic Cluster States
}

\author{
Pei Wang \\ Danjiangkou, Hubei, China \\ B.S. Physics, Fudan University, 2010 \\ A Dissertation presented to the Graduate Faculty \\ of the University of Virginia in Candidacy for the degree of \\ Doctor of Philosophy \\ Department of Physics \\ University of Virginia \\ May 2016
}




\section{Acknowledgments}

First, I must express my gratitude to my advisor, Prof. Olivier Pfister. Prof. Pfister is a fine person and a resourceful mentor, it is my honor to work in his research group. He is alway optimistic and passionate about physics. I thank him for being extremely patient with me, for always being amiable with a great sense of humor. I thank him for giving me a lot of freedom to explore things by myself. What I have learned from him is far beyond research, I am really grateful that I can work with such a nice advisor during my $\mathrm{PhD}$.

I thank our genius collaborator Dr. Nicolas C. Menicucci. Most of my research is inspired by his impactful works. His theories are consistently precise and elegant. I must thank him for all the brilliant ideas. It was a great experience to work with him.

I am very thankful for all of my lab mates. Reihaneh Shahrokhshahi is a passionate researcher and a great leader of our OSA student chapter. I thank her for her encouragements on my research and getting me involved in OSA chapter. Moran Chen was my mentor for the experiment. She is always patient with the (usually exciting, but "sometimes") tedious experiments. I have learned a lot from her beyond the experimental skills. Niranjan Sridhar is a talented researcher and a great communicator, we had a lot of inspiring discussions about physics and future career possibilities. Wenjiang Fan and me worked together on an interesting experiment, 
our collaboration naturally extends to basketball and soccer. Writing this paragraph reminds me the memorable days when we worked together in the lab: lasers are on, the room is dark; TES is chirping, from day to night. I must thank all of them for their generous helps, we had a great time. I also thank Rajveer Nehra and Ben Godek, I am sure they will continue the momentum and make more exciting progresses.

I thank my committee members for their time to review my research summaries and thesis, and for their great questions and feedbacks. I thank all my course instructors for the great lectures. I thank all the physics department secretaries for always being nice and helpful. I thank the physics department staff members for their helps on the purchases, book reservations and other requests.

I thank my parents for their unconditional supports at all times. Being at a distant country of a different culture, my families always give me enormous power to confront all kind of challenges. I thank my wife, Tiantian Ren, for all the happy moments we have during the past six years, for broadening my vision, for giving me a new perspective of the meaning of life. And of course, thank you for all the delicious food that charged my power up in the best possible way.

Finally, I thank all of you for being with me during this fruitful voyage. May the entanglement be with us. 


\section{Abstract}

Quantum computing promises exponential speedup for particular computational tasks, such as integer factoring [1], which bears importance for encryption technology, and quantum simulation [2], which holds vast scientific potential. Comparing with the traditional circuit-based quantum computers, one-way quantum computers are experimentally appealing because it requires only local measurements on an entan-

gled resource called a cluster state $[3]$. In this thesis, I first review the fundamental theory of one-way quantum computing and its experimental implementation using the quantum optical frequency comb. After that, I present two novel schemes for generating ultra-scalable cluster states with rich structures that can be used for universal quantum computing 4,5$]$. 


\section{Contents}

Acknowledgments . . . . . . . . . . . . . . . . i

Abstract ................................. ii

List of Figures . . . . . . . . . . . . . . . . . . . . vi vi

\begin{tabular}{lll}
\hline & Introduction & 1
\end{tabular}

2 Qubits, Qumodes and Cluster States 5

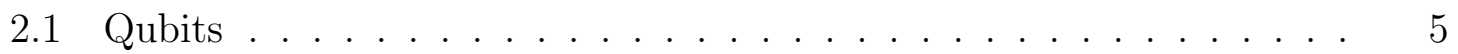

2.2 Qumodes . . . . . . . . . . . . . . . . . . . . . . . . . 8

2.3 Cluster States . . . . . . . . . . . . . . . . . . . . . . . . . . . . 11

2.3 .1 Cluster State Basics . . . . . . . . . . . . . . . . . . . . 11

2.3 .2 The Stabilizer Formalism . . . . . . . . . . . . . . . . . . . . . 14

2.3 .3 Cluster states with Stabilizer Formalism . . . . . . . . . . . . 16

2.3 .4 Ideal Qumodes . . . . . . . . . . . . . . . . . . 17

2.3 .5 Practical qumodes . . . . . . . . . . . . . . . . . . 21

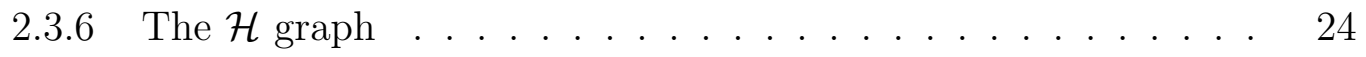

2.4 From $\mathcal{H}$ graph to $\mathbf{Z}$ graph . . . . . . . . . . . . . . . . . . . . . . . . 24

$2.4 .1 \quad$ Heisenberg picture . . . . . . . . . . . . . . . . . . . 25

2.4 .2 Schrödinger picture . . . . . . . . . . . . . . . . . . . . 29

2.4 .3 When $\mathrm{G}$ is not self-inverse . . . . . . . . . . . . . . . . . . . 33

2.5 Summary . . . . . . . . . . . . . . . . . . . . . . 40

3 The Optical Parametric Oscillator 41

3.1 Optical Cavity . . . . . . . . . . . . . . . . . . . . . . . 41

3.2 Nonlinear Optics . . . . . . . . . . . . . . . . . . . . . . 44

$3.2 .1 \quad$ Introduction to nonlinear optics . . . . . . . . . . . . . . 44

3.2 .2 Second-Harmonic Generation . . . . . . . . . . . . . . . . 47

3.2 .3 Phasematching $\ldots \ldots \ldots$. . . . . . . . . . . . . . . . . . . . . . 49 
3.2 .4 Quasi-phasematching bandwidth of PPKTP . . . . . . . 51

3.3 The Optical Parametric Oscillator . . . . . . . . . . . . . . . . 57

$3.3 .1 \quad$ OPO dispersion $\ldots \ldots \ldots \ldots \ldots \ldots$

3.4 Conclusion . . . . . . . . . . . . . . . . . . . . . . 65

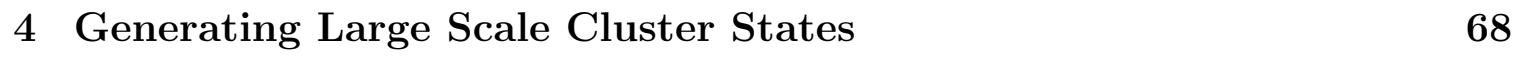

4.1 The Hypercubic Cluster States . . . . . . . . . . . . . . . . . . . . 68

4.1 .1 Two-mode squeezed states generation . . . . . . . . . . . 69

$4.1 .2 \quad$ Macronode lattice setup . . . . . . . . . . . . . . . . . 72

4.1 .3 Macronode lattice entanglement . . . . . . . . . . . . . . 77

4.1 .4 State verification . . . . . . . . . . . . . . . . . . . . . 81

4.1 .5 Conclusion for the hypercube proposal $\ldots \ldots \ldots \ldots$

4.2 The Time-Frequency Domain Cluster States . . . . . . . . . . . . 84

4.2 .1 State generation . . . . . . . . . . . . . . . . . . 84

4.2 .2 Quantum computing . . . . . . . . . . . . . . . . 87

$4.2 .3 \quad$ Other Types of Cluster States $\ldots \ldots \ldots$

4.2 .4 The Self-inverse Property _. . . . . . . . . . . . . . . . . . 89

4.2 .5 Conclusion of the hybrid cluster states proposal . . . . . . . 92

$\begin{array}{lll}5 \text { Conclusion } & 96\end{array}$ 


\section{List of Figures}

2.1 Comparison between qubits and qumodes. . . . . . . . . . . . . 10

2.2 Examples of graph states. . . . . . . . . . . . . . . . . . . . . . . . . 12

3.1 Cavity transmittance versus input frequency. . . . . . . . . . . . . . . 43

3.2 Generated field intensity at different phasematching conditions. . . . 49

3.3 Quasi-phasematching function. . . . . . . . . . . . . . . . 53

3.4 SFG Experimental setup. . . . . . . . . . . . . . . . . . . . . 54

3.5 Experimental and theoretical phasematching curve. . . . . . . . . . 56

3.6 Pair generation by single pump. . . . . . . . . . . . . . . . 58

3.7 The dispersion effect of the OPO. . . . . . . . . . . . . . . . . . . . 60

3.8 Frequency shift caused by dispersion. . . . . . . . . . . . . . . . . 62

3.9 Average frequency shift caused by dispersion. . . . . . . . . . . . 65

4.1 TMSSs generation with two pumps. . . . . . . . . . . . . . . . . 71

4.2 TMSS arrangement. . . . . . . . . . . . . . . . . . . . . . . . 73

4.3 Generating multiple copies of lattice cluster states. . . . . . . . . . . 76

4.4 Hypercube experimental setup. . . . . . . . . . . . . . . . . 79

4.5 Hypercube macronode interferometry. . . . . . . . . . . . . . . . . . . 83

4.6 BSL cluster state experimental setup. . . . . . . . . . . . . . . . 86

4.7 Two MBQC protocols on the BSL cluster state. . . . . . . . . . . . . 88

4.8 Delay every three or four nodes. . . . . . . . . . . . . . . . . . 94

4.9 Self-inverse check. . . . . . . . . . . . . . . . . . . . . . . . . . . . . . . . . . . . 95 


\section{Chapter 1}

\section{Introduction}

Quantum computing (and quantum information) is an interdisciplinary research field where physics, engineering and computer science converge [1,2]. This field is developing rapidly and is attracting large amount of attention, both in academia and in industry [6].

What is quantum computing? A short definition of quantum computing is: computing by using the laws of quantum mechanics. The concept of quantum computing was first introduced by Richard Feynman [2]. In 1982, Feynman proposed building a computer to simulate the evolution of a quantum system, where the computer itself follows laws of quantum mechanics and tracks the system naturally, so it can simulate the system exponentially faster 1 than using a classical computer. For example, a lattice of $N$ spins has $2^{N}$ possible states, the problem size for a classical computer is $2^{N}$, but only $N$ for a quantum computer because the quantum evolution of the $N$ spins can be captured directly.

\footnotetext{
${ }^{1}$ This is a vague term, when people say "A is exponentially faster than B", usually it mean $\mathrm{B}$ takes exponential time $\left(O\left(2^{N}\right)\right)$ of $N$, while A only takes polynomial time $\left(O\left(N^{k}\right)\right)$. The Big $\mathrm{O}$ notation $O(f(N))$ indicates the asymptotic upper bound of computational complexity.
} 
In 1994, Peter Shor demonstrated that quantum computers can factorize integers exponentially faster than classical computers [1]. This attracted attention beyond academia, because the most widely used encryption method, known as RSA [7], is based the fact that factorizing large integers is very hard 2. A quantum computer can factorize integers efficiently, so the currently encrypted information won't be safe anymore once quantum computers have been built. The discovery opened a new research area in cryptography. Besides Shor's discovery, a few other quantum algorithms were also demonstrated [8].

Due to the impact on both fundamental physics, computer science, and practical applications, and the progress in experimental technologies, quantum computing has been a very active and expanding research field.

With enough motivation, we now look at how a quantum computer works. Like classical computers, a quantum computer needs to have information units and perform operations over the units. For a certain computing task, it takes the input information, performs logic operations on the input, and collects the output information as the result. The information units are known as quantum bits, or qubits for short, as opposed to classical bits. The operations are known as quantum logic gates, as opposed to classical logic gates. There are several different ways of implementing the information units and the operations.

The information units can be discrete variables (DV) or continuous variables (CV). If the unit is a qubit, i.e., a two-level system, then it's of the DV kind. In clas-

\footnotetext{
${ }^{2}$ No algorithm that has been published can factor all integers in polynomial time.
} 
sical computers, the value of the bit can only be 0 or 1 . However, for a quantum computer, the qubit can also be a superposition of 0 and 1 , such as: $|\phi\rangle=\alpha|0\rangle+\beta|1\rangle$. Moreover, there could be quantum entanglement between the qubits. If the unit is in a d-level system, then it's still in the DV regime, the unit is called qudit. If the unit is continuous, then it's a CV, and the unit is often called qumode. Those three types of encoding share some similar properties, which we will discuss in the next Chapter. In this thesis, I will focus on the continuous-variable encoding.

As for the operations, there are two mainstream implementations: the circuitbased model [9] and the measurement-based model [10]. The traditional circuit-based model is similar to the classical computing case, but the "circuits" are now quantum circuits, featuring qubits and quantum gates. Another difference between a classical and a quantum computer is, for a quantum computer, usually one needs to perform some quantum measurements on the system to make the quantum algorithm work. On the contrary, the measurement-based model has no classical analogue. This model pre-embeds quantum gates in an entanglement source, known as a cluster state [10]. After the preparation, the input information is first entangled with the cluster state, then all quantum logic gates are implemented by single-qubit measurements on the whole entangled state [10]. During the measurements, the cluster state is usually destroyed, and the information flows one-way from the input to output, so this model is also known as one-way quantum computing [10].

After presenting the abstract architecture of quantum computers, we now look at 
the physical implementation. There are a lot of different candidate systems: semiconductors [11], superconductors [6], trapped ions [12], optics [13, 14], to name a few. Basically, anything that demonstrates observable quantum behaviors and is manipulable can be a candidate for quantum computing. In this thesis, I will focus on optical one-way quantum computing with continuous variables. That is, the information is encoded in continuous variables, the operation is done in a measurement-based way, and the system is implemented by quantum optics.

The thesis is organized as the following:

In Chapter 2, I introduce the basic concepts for quantum computing, we look into details of qubits and qumodes, and then focus on the cluster state, which is the heart of a one-way quantum computer.

In Chapter 3, I talk about the components and functionality of the optical parametric oscillator (OPO). I will also talk about the limitations of the OPO.

In Chapter 4, I demonstrate how to combine the techniques in previous chapters to generate large scale entangled source for universal quantum computing. In particular, I will demonstrate two novel schemes proposed in Ref. [4] and Ref. [5] in detail.

In Chapter 5, I summarize this thesis and discuss some future possibilities. 


\section{Chapter 2}

\section{Qubits, Qumodes and Cluster States}

In this chapter, I cover in more detail about the building blocks of a measurementbased quantum computer: qubits, qumodes and cluster states.

\section{$2.1 \quad$ Qubits}

For a system in a two-dimensional Hilbert space of computational basis $\{|0\rangle,|1\rangle\}$ , a general state can be written as:

$$
|\psi\rangle_{\theta, \phi}=\cos \frac{\theta}{2}|0\rangle+\sin \frac{\theta}{2} e^{i \phi}|1\rangle
$$

This state can be represented using a Bloch sphere, where $\theta$ and $\phi$ are the spherical coordinates. The two states in the basis $\{|0\rangle,|1\rangle\}$ can also be written in canonical vector form:

$$
|0\rangle=\left(\begin{array}{l}
0 \\
1
\end{array}\right),|1\rangle=\left(\begin{array}{l}
1 \\
0
\end{array}\right)
$$

With the state defined, I introduce some useful unitaries: the Pauli operators, 
the rotation operator, the Hadamard operator. I start with the Pauli operators. The Pauli operators can be written as Pauli matrices in the qubit representation:

$$
\hat{X}=\left(\begin{array}{cc}
0 & 1 \\
1 & 0
\end{array}\right), \hat{Y}=\left(\begin{array}{cc}
0 & -i \\
i & 0
\end{array}\right), \hat{Z}=\left(\begin{array}{cc}
1 & 0 \\
0 & -1
\end{array}\right)
$$

Note that these matrices are both unitary and Hermitian. We can check what they do to the states. Applying these operators to the basis states, we get:

$$
\hat{X}|s\rangle=|\bar{s}\rangle, \hat{Z}|s\rangle=(-1)^{s}|s\rangle
$$

where $s \in\{0,1\}$, and $\overline{0}, \overline{1}=1,0$ respectively. We can see that $\hat{X}$ flips the qubit, $\hat{Z}$ adds a phase to the qubit. The Pauli $\hat{Z}$ and $\hat{X}$ are the generators of the Pauli group.

Now we can define the rotation operator using the Pauli operators:

$$
\hat{R}_{\mathbf{s}}(\theta)=e^{-i \frac{\theta}{2} \mathbf{s} \cdot \boldsymbol{\sigma}}
$$

where $\mathbf{s}$ is the unit vector around which the rotation is performed, and $\boldsymbol{\sigma}=(\hat{X}, \hat{Y}, \hat{Z})^{\mathrm{T}}$. For example, a rotation around $\hat{Z}$ by $\theta$ will be $\hat{Z}_{\theta}:=\hat{R}_{(0,0,1)}=e^{-i \theta \hat{Z} / 2}=\cos \frac{\theta}{2} \mathbb{1}+$ $i \sin \frac{\theta}{2} \hat{Z}$. By applying the previous formula, or in the general case, the Baker-CampbellHausdorff (BCH) formulas, we can get:

$$
\begin{aligned}
& \hat{Z}_{\theta}^{\dagger} \hat{X} \hat{Z}_{\theta}=\hat{X} \cos \theta-\hat{Y} \sin \theta \\
& \hat{Z}_{\theta}^{\dagger} \hat{Y} \hat{Z}_{\theta}=\hat{X} \sin \theta+\hat{Y} \cos \theta \\
& \hat{Z}_{\theta}^{\dagger} \hat{Z} \hat{Z}_{\theta}=\hat{Z}
\end{aligned}
$$


Just as the qubit can be represented by the Bloch sphere, single-qubit unitaries can be viewed as rotation on the Bloch sphere. So all the single-qubit unitaries can be written as a rotation operator.

I now introduce the conjugate basis of $\{|0\rangle,|1\rangle\}$ :

$$
| \pm\rangle=\frac{1}{\sqrt{2}}(|0\rangle \pm|1\rangle)
$$

It has the similar behaviors under Pauli operators:

$$
\hat{X}| \pm\rangle= \pm| \pm\rangle, \hat{Z}| \pm\rangle=|\mp\rangle
$$

We can see $\hat{X}$ adds a phase to the qubit, $\hat{Z}$ flips the qubit. The Hadamard gate $\hat{H}$ transforms one basis to the other:

$$
\hat{H}|0\rangle=|+\rangle, \hat{H}|1\rangle=|-\rangle
$$

We can also write it in the matrix form:

$$
\hat{H}=\frac{1}{2}\left(\begin{array}{cc}
1 & 1 \\
1 & -1
\end{array}\right)
$$

Having introduced some useful operators, for quantum computing purpose, I now define a special group of operators, known as the Clifford group. The Clifford group is a group formed by single-qubit unitaries that transform Pauli operators into Pauli operators. For example, Pauli operators, Hadamard gate, the $\hat{Z}_{\pi / 2}$ are in the Clifford group, but $\hat{Z}_{\pi / 4}$ is not (we can easily check this by using Eq. 2.6.).

If any unitary operations can be approximated to arbitrary accuracy by only 
involving a certain set of quantum gates, then this set of quantum gates is called the universal set [9]. A universal set of gates is usually chosen to be: $\left\{\hat{H}, \hat{Z}_{\pi / 2}, \hat{Z}_{\pi / 4}, \hat{C}_{Z}\right\}$. This set has three single-qubit gates and one two-qubit gate. It's proved that all gates can be decomposed into single-qubit gates and two-qubit gates (same as the classical computing case). So if the three single-qubit gates can perform general single-qubit operations, then this set is clearly universal. The general single-qubit operation is a rotation for arbitrary angle around arbitrary axis. It's not obvious that how this discrete set is able to produce rotations at all angles (which is continuous), but it is beyond the scope of this thesis. Interested readers can check the proof in Ref. [9].

\subsection{Qumodes}

A qumode is a quantized harmonic oscillator. The quadrature operators, dimensionless analogues of position and momentum, are defined in terms of the creation $\left(\hat{a}^{\dagger}\right)$ and annihilation $(\hat{a})$ operators:

$$
\begin{aligned}
& \hat{q}=\frac{1}{\sqrt{2}}\left(\hat{a}+\hat{a}^{\dagger}\right) \\
& \hat{p}=\frac{1}{i \sqrt{2}}\left(\hat{a}-\hat{a}^{\dagger}\right)
\end{aligned}
$$

From the commutator: $\left[\hat{a}, \hat{a}^{\dagger}\right]=1$, we find the commutator of the quadrature operators: $[\hat{q}, \hat{p}]=i$. The Hamiltonian of a qumode can be written as:

$$
\begin{aligned}
\hat{H} & =\hbar \omega\left(\hat{a}^{\dagger} \hat{a}+\frac{1}{2}\right) \\
& =\frac{\hbar \omega}{2}\left(\hat{q}^{2}+\hat{p}^{2}\right)
\end{aligned}
$$


The eigenstates ${ }^{1}$ of $\hat{q}$ and $\hat{p}$ are the analogues of qubit basis $\{|0\rangle,|1\rangle\}$ and $\{|+\rangle,|-\rangle\}$ respectively. The reason may not be obvious at this point but it will be clear later.

$|q\rangle_{q}$ and $|p\rangle_{p}$ are related by the Fourier transformation:

$$
\begin{aligned}
& |q\rangle_{q}=\frac{1}{\sqrt{2 \pi}} \int_{-\infty}^{\infty} e^{-i q p}|p\rangle_{p} d p \\
& |p\rangle_{p}=\frac{1}{\sqrt{2 \pi}} \int_{-\infty}^{\infty} e^{i q p}|q\rangle_{q} d q
\end{aligned}
$$

The Fourier transformation can also be written as $\hat{F}|x\rangle_{q}=|x\rangle_{p}$. Because the Hadamard gate transforms one basis into the other, the Hadamard gate on qubits is the analogue of the Fourier transformation on qumodes.

The Pauli operators on qubits also have analogue on qumodes. We define the Weyl-Heisenberg (WH) operators as:

$$
\begin{aligned}
& \hat{X}(s) \equiv e^{-i s \hat{p}} \\
& \hat{Z}(s) \equiv e^{i s \hat{q}}
\end{aligned}
$$

with this we can see:

$$
\begin{aligned}
& \hat{X}(s)|q\rangle=|q+s\rangle_{q} \\
& \hat{Z}(s)|q\rangle=e^{i s q}|q\rangle_{q}
\end{aligned}
$$

Compare this with Eq. 2.4, we can see the Weyl-Heisenberg $\hat{X}$ and $\hat{Z}$ to qumodes is the analogue of the Pauli $\hat{X}$ and $\hat{Z}$ to qubits.

\footnotetext{
${ }^{1}$ In this thesis, the the two quadrature eigenstates are denoted by $|\cdot\rangle_{q}$ and $|\cdot\rangle_{p}$ respectively.
} 
Similar to the qubit Clifford group, the qumode Clifford group is a group of singlequmode unitaries that transform WH operators into WH operators. The unitaries in the Clifford group are also called Gaussian unitaries. The unitaties I have covered so far are all Gaussian unitaries. One example of the non-Gaussian unitaries is the cubic phase gate $\hat{D}_{3}(t)=\exp \left(i t \hat{q}^{3}\right)$. Because:

$$
\hat{D}_{3}(t) \hat{X}(s) \hat{D}_{3}^{\dagger}(t)=\hat{X}(s) \hat{Z}\left(6 t s^{2}\right) e^{3 i s t \hat{q}^{2}}
$$

The right-hand side is not a multiplication of WH operators. Table 2.1 shows a comprehensive comparison between qubits and qumodes.

\section{Discrete Variables (DV) Continuous Variables (CV)}

\begin{tabular}{ccc}
\hline Basis & $\{|s\rangle, s \in\{0,1\}\}$ & $\{|q\rangle, q \in \mathbb{R}\}$ \\
\hline General states & $|\psi\rangle=\alpha|0\rangle+\beta|1\rangle$ & $|\psi\rangle=\int_{-\infty}^{\infty} \psi(q)|q\rangle d q$ \\
\hline Conjugate basis & $| \pm\rangle=|0\rangle \pm|1\rangle$ & $|p\rangle=\frac{1}{\sqrt{2 \pi}} \int_{-\infty}^{\infty} e^{i p q}|q\rangle d q$ \\
\hline $\begin{array}{c}\text { Bipartite } \\
\text { maximally }\end{array}$ \\
$\begin{array}{c}\text { Entangled states } \\
\text { Single-qubit }\end{array}$ & $\left|B_{00}\right\rangle_{12}=|00\rangle_{12}+|11\rangle_{12}$ & $|E P R\rangle_{12}=\int_{-\infty}^{\infty}|q q\rangle_{12} d q$ \\
$\begin{array}{c}\text { (qumode) gates } \\
\text { Pauli } \hat{Z}\end{array}$ & WH $\hat{Z}(\varpi)=e^{i \varpi \hat{q}}$ \\
\hline $\begin{array}{c}\text { Two-qubit } \\
\text { (qumode) gate }\end{array}$ & $\hat{C}_{z}=\hat{Z}_{2}^{s_{1}}, s_{1} \in\{0,1\}$ & $\hat{C}_{z}=e^{i \hat{q}_{1} \hat{q}_{2}}=\hat{Z}_{2}\left(\hat{q}_{1}\right)$
\end{tabular}

Table 2.1: Comparison between qubits and qumodes. 


\subsection{Cluster States}

Now we are ready to look at details about the cluster state, which is the heart of a measurement-based quantum computer. In this Section, I first introduce the qubit cluster states, and then talk about the stabilizer formalism for representing the cluster states, and then the qumode cluster states.

\subsubsection{Cluster State Basics}

Let's start with the $n$-qubit state $|+\rangle^{\otimes N}$. This state can be represented by a graph of $n$ isolated vertices. Now we apply controlled- $Z$ gates between some pairs of qubits, then the final state can be represented by a graph where the vertices are qubits and edges are the entanglement between qubits. This state is called graph state. The structure of the graph state depends on where the controlled- $Z$ gates are applied. If the graph is a $d$-dimensional square-grid lattice, then the state is called the cluster stat $\AA^{2}$,

Let's look at some examples. The state in Fig. 2.2(a) is:

$$
C Z_{12}|+\rangle_{1}|+\rangle_{2}=\frac{1}{\sqrt{2}}(|0+\rangle+|1-\rangle)
$$

If we apply a Hadamard gate on the second qubit, the state becomes: $(|00\rangle+$ $|11\rangle) / \sqrt{2}$, which is a maximally entangled state in two dimensional Hilbert space, also known as the Bell state [9].

\footnotetext{
${ }^{2}$ The community has different definitions of cluster states, I adopt this definition in this thesis.
} 
(a)

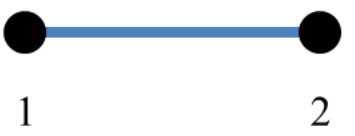

(c)

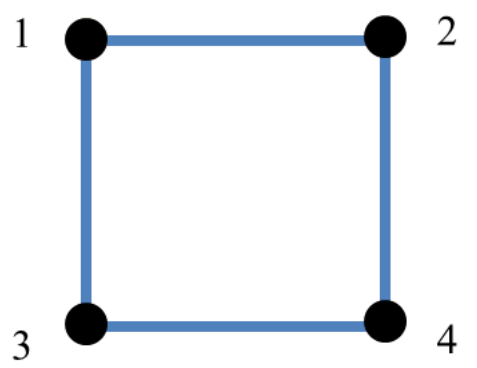

(b)

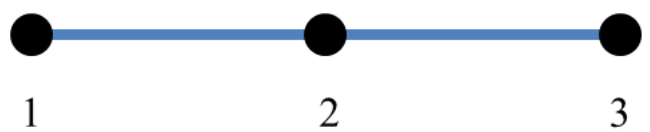

(d)

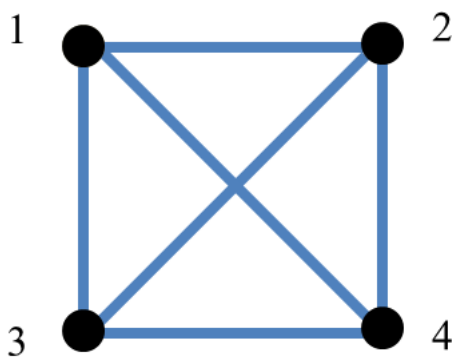

Figure 2.2: Examples of graph states.

The state in Fig. 2.2(b) is:

$$
\begin{aligned}
C Z_{12} C Z_{23}|+\rangle_{1}|+\rangle_{2}|+\rangle_{3} & =\frac{1}{2}(|00+\rangle+|01-\rangle+|10+\rangle-|11-\rangle) \\
& =\frac{1}{\sqrt{2}}(|+0+\rangle+|-1-\rangle)
\end{aligned}
$$

If we apply a Hadamard gate on the second qubit, the state becomes: $\frac{1}{2}(|+++\rangle+$ $|---\rangle)$, which is known as a Greenberger-Horne-Zeilinger (GHZ) state. This graph can be viewed as a "star graph", in which all nodes are linked to a single center node. In this particular case, node 2 is the center. One can prove that all star graphs are LU-equivalent to GHZ states, where LU-equivalent means equivalent after applying local unitaries $[15]$. 
The state in Fig. 2.2.(c) is:

$$
\begin{aligned}
& C Z_{12} C Z_{13} C Z_{24} C Z_{34}|+\rangle_{1}|+\rangle_{2}|+\rangle_{3}|+\rangle_{4} \\
= & \frac{1}{2 \sqrt{2}}(|000+\rangle+|100-\rangle+|001-\rangle+|101+\rangle \\
& +|010+\rangle-|110-\rangle-|011-\rangle+|111+\rangle) \\
= & \frac{1}{2}(|0+0+\rangle+|1+1+\rangle+|0-1-\rangle+|1-0-\rangle)
\end{aligned}
$$

As we can see as the number of qubits increases, the final state becomes more complicated. If I write everything in the $\{|0\rangle,|1\rangle\}$ basis, it has 16 terms.

The state in Fig. 2.2(d) is:

$$
\begin{aligned}
& C Z_{12} C Z_{13} C Z_{24} C Z_{34} C Z_{14} C Z_{23}|+\rangle_{1}|+\rangle_{2}|+\rangle_{3}|+\rangle_{4} \\
= & \frac{1}{2 \sqrt{2}}(|000+\rangle+|010-\rangle-|101+\rangle-|111-\rangle \\
& +|001-\rangle-|011+\rangle+|100-\rangle-|110+\rangle)
\end{aligned}
$$

Ref. [15] proved the final state above is LU-equivalent to a GHZ state. In general, a graph state with complete graph is LU-equivalent to a GHZ state [15].

From these examples, we can clearly see that as the number of qubits increases, the state vector soon becomes exponentially larger ( $2^{N}$ terms for $N$ qubits). Also, by looking at the final state vector, it's very hard to know what the graph is. Fortunately, there's a better way to describe the state, that is to use the stabilizer formalism. 


\subsubsection{The Stabilizer Formalism}

Let $S$ be a group of operators. If an operator $\hat{K} \in S$ satisfies $\hat{K}|\psi\rangle=\psi$, then we say $|\psi\rangle$ is stabilized by $\hat{K}$. If all the state vectors from a vector space $V_{s}$ are stabilized by every operator $\hat{K}$ from a group $S$, then $S$ is called a stabilizer of $V_{s}[9]$. For example, $S=\{I, X\}$ is a stabilizer of $V_{s}=\{|+\rangle\}, S=\left\{I, Z_{1} Z_{2}, Z_{2} Z_{3}, Z_{1} Z_{3}\right\}$ is a stabilizer of $V_{s}=\{|000\rangle,|111\rangle\}$.

A group can always be represented by its generators, which are independent elements that generate the group by applying group multiplication. For example, $\left\{Z_{1} Z_{2}, Z_{2} Z_{3}\right\}$ are the generators of group $S=\left\{I, Z_{1} Z_{2}, Z_{2} Z_{3}, Z_{1} Z_{3}\right\}$. I can then denote the group by its generators: $S=\left\langle Z_{1} Z_{2}, Z_{2} Z_{3}\right\rangle$, and use $\langle\ldots\rangle$ to indicate that these are the generators instead of an enumeration of all elements.

The group of most interest is the Pauli group $G_{n}$ on $n$ qubits, which is a group of all $n$-fold tensor products of Pauli operators [9], with multiplicative factors of $\pm 1, \pm i$. If $S=\left\langle g_{1}, \ldots, g_{n-k}\right\rangle$, where $g_{1}, \ldots, g_{n-k}$ are independent and commuting elements from $G_{n}$, then $V_{s}$ that is stabilized by $S$ is a $2^{k}$ dimensional vector space [9]. For example, in the case $n=3$ and $S=\left\langle Z_{1} Z_{2}, Z_{2} Z_{3}\right\rangle$, the vector space $V_{s}=\{|000\rangle,|111\rangle\}$ is s $2^{(3-2)}=2$ dimensional vector space. This tells us that a certain stabilizer corresponds to a particular set of states, so we can use stabilizers to describe the states.

This is good, but if we only look at the example above where $S=\left\langle Z_{1} Z_{2}, Z_{2} Z_{3}\right\rangle$, $V_{s}=\{|000\rangle,|111\rangle\}$, it's not very impressive that we need three operators to represent two states. However, if we apply some unitaries on the states, then the resulting 
state can have up to 8 terms, yet we will still only have three operators in the stabilizer group, as I will show soon. In general, for $N$ qubits, the state vector has $2^{N}$ terms, the stabilizer group only has $N$ generators.

We now discuss how to apply unitaries in the stabilizer formalism. For $g \in S$ and $|\psi\rangle \in V_{s}$, applying $\hat{U}$ on the state $|\psi\rangle$ gives: $\hat{U}|\psi\rangle=\hat{U} g \hat{U}^{\dagger} \hat{U}|\psi\rangle$, so $\hat{U} V_{s}$ is stabilized by the group $\left\{\hat{U} g \hat{U}^{\dagger} \mid g \in S\right\}$. Now we can see for applying unitaries, we just need to transform the group elements in the stabilizer, which is usually very easy to do for Pauli operators, but much harder to keep track of if we use the state vectors.

The last piece in this formalism is the measurement. In here we only cover Pauli measurement $g$. There are three different cases:

(1) If $g$ commutes with all elements in $S=\left\langle g_{1}, \ldots, g_{n-k}\right\rangle$, then $\pm g$ is in the stabilizer, we just need to consider the measurement outcome, the new stabilizer is $S=\left\langle \pm g, g_{2}, \ldots, g_{n-k}\right\rangle$

(2) If $g$ doesn't commute with $g_{1}$, then we just kick $g_{1}$ out and add the measurement outcome, so finally $S=\left\langle \pm g, g_{2}, \ldots, g_{n-k}\right\rangle$.

(3) If $g$ doesn't commute with $g_{1}$ and $g_{2}$, we kick both of them out and add $\pm g$, but we need one more operator that commutes with everybody in the group. One can easily check that $g_{1} g_{2}$ works. So finally the stabilizer becomes $S=\left\langle \pm g, g_{1} g_{2}, \ldots, g_{n-k}\right\rangle$. We can use the same principles for the general cases.

At this point, I have already showed how to represent states using stabilizer formalism, and how to handle unitaries and measurements. We are ready to apply this 
formalism to study the properties of the cluster states.

\subsubsection{Cluster states with Stabilizer Formalism}

Now given any graphs, we know exactly what the corresponding state is: we just need to follow the above procedure to construct the graph from the beginning. But what happens when we apply unitaries to the state? What about measurements? Of course we can write down the state vector for the graph state, and check what happens after those operations. However, this method is very hard and inefficient, as we pointed out in the previous section. We have a better choice: use the stabilizer formalism to represent the graph states.

We start with the initial state. The stabilizer for the initial state (before any controlled- $Z$ has applied) is: $S=\left\langle\left\{X_{i} \mid 0 \leq i \leq n\right\}\right\rangle$. A controlled- $Z$ between 1 and 2 (1 is the control and 2 is the target) will transform $X_{1}$ into $X_{1} Z_{2}$, and leave all other Pauli operators unchanged. So the final stabilizer is:

$$
S=\left\langle\left\{X_{i} \prod_{j \in N(i)} Z_{j} \quad \mid \quad 0 \leq i \leq n\right\}\right\rangle
$$

where $N(i)$ represents the neighbors of node $i$ in the graph. Using this expression, one can easily obtain the stabilizer given a graph, by just looking at every node and its neighbors.

Now we have the stabilizer for the graph, we can use the rules in previous section to figure out what happens after applying unitaries. I refer interested readers to find this out in Ref. [15]. 
A very useful property of the graph state is the graph can be reshaped by applying measurements. Measuring the $Z$ of node $i$ will remove all the links to node $i$ while leaving the rest part of the graph unchanged. To see this, we apply the stabilizer formalism for measurements introduced in the previous section. The measurement $Z_{i}$ commutes with all the elements in the group except for $X_{i} \prod_{j \in N(i)} Z_{j}$, so this one is kicked out and replaced by $\pm Z_{i}$. By multiplying $\pm Z_{i}$ to the all the other elements, the $Z_{i}$ term in all the other elements is replaced by the measurement outcome \pm 1 . Now we can see that node $i$ is now isolated from the rest of the graph, but other nodes are not affected, except for $i$ 's neighbors get a multiplicative factor \pm 1 , which can be fixed by applying local Pauli operators ${ }^{3}$. Measuring $X$ or $Y$ has more complicated effect on the graph, interested readers can take a look at Ref. [15].

\subsubsection{Ideal Qumodes}

Now we consider qumodes. The stabilizer formalism translates well to qumodes if we replace everything from qubits to qumodes according to Fig. 2.1. That is, we start from the momentum zero state of all qumodes: $|0\rangle_{p}^{\otimes N}$, then apply controlled- $Z$ to entangle the qumodes. Let's look at the same graphs in Fig. 2.2, with all nodes being qumodes instead of qubits.

\footnotetext{
${ }^{3}$ This process is known as feedforward.
} 
The state in Fig. 2.2(a) ist

$$
\begin{aligned}
C Z_{12}|0\rangle_{1 p}|0\rangle_{2 p} & =e^{i \hat{q}_{1} \hat{q}_{2}} \int_{-\infty}^{+\infty} d q_{1}\left|q_{1}\right\rangle_{1 q} \int_{-\infty}^{+\infty} d q_{2}\left|q_{2}\right\rangle_{2 q} \\
& =\int_{-\infty}^{+\infty} d q|q\rangle_{1 q}|q\rangle_{2 p}
\end{aligned}
$$

Note that the state of qumode 2 is in the $p$ basis. If we apply a Fourier transform on the second qumode, the state becomes:

$$
\int_{-\infty}^{+\infty} d q|q\rangle_{1 q}|q\rangle_{2 q}=\int_{-\infty}^{+\infty} d p|p\rangle_{1 p}|-p\rangle_{2 p}
$$

This is a maximally entangled state for two qumodes, also known as the EinsteinPodolsky-Rosen (EPR) state [16]. This state is the analogue of the Bell states in qubits.

The state in Fig. 2.2(b) is:

$$
\begin{aligned}
C Z_{12} C Z_{23}|0\rangle_{1 p}|0\rangle_{2 p}|0\rangle_{3 p} & =\int_{-\infty}^{+\infty} \int_{-\infty}^{+\infty} \int_{-\infty}^{+\infty} d q_{1} d q_{2} d q_{3} e^{i q_{1} q_{2}} e^{i q_{2} q_{3}}\left|q_{1}\right\rangle_{1 q}\left|q_{2}\right\rangle_{2 q}\left|q_{3}\right\rangle_{3 q} \\
& =\int_{-\infty}^{+\infty} d q|q\rangle_{1 p}|q\rangle_{2 q}|q\rangle_{3 p}
\end{aligned}
$$

If we apply a Fourier transform on the second qumode, the state becomes a (CV) GHZ state. Same as in the qubit case, all star graphs are GHZ states (with a local Fourier transform applied on the center node).

\footnotetext{
${ }^{4}$ These states can't be normalized, I will address this later.
} 
The state in Fig. 2.2(c) is:

$$
\begin{aligned}
& C Z_{12} C Z_{13} C Z_{24} C Z_{34}|0\rangle_{1 p}|0\rangle_{2 p}|0\rangle_{3 p}|0\rangle_{4 p} \\
= & \int_{-\infty}^{+\infty} \int_{-\infty}^{+\infty} d q d q^{\prime}|q\rangle_{1 p}\left|q+q^{\prime}\right\rangle_{2 p}|q\rangle_{3 q}\left|q+q^{\prime}\right\rangle_{4 p}
\end{aligned}
$$

The state in Fig. 2.2(d) is:

$$
\begin{aligned}
& C Z_{12} C Z_{13} C Z_{24} C Z_{34} C Z_{14} C Z_{23}|0\rangle_{1 p}|0\rangle_{2 p}|0\rangle_{3 p}|0\rangle_{4 p} \\
= & \int_{-\infty}^{+\infty} \int_{-\infty}^{+\infty} \int_{-\infty}^{+\infty} d q d q^{\prime} d q^{\prime \prime} e^{i q q^{\prime}} e^{i\left(q+q^{\prime}\right) q^{\prime \prime}}|q\rangle_{1 q}\left|q^{\prime \prime}\right\rangle_{2 q}|q\rangle_{3 q}\left|q+q^{\prime}+q^{\prime \prime}\right\rangle_{4 p}
\end{aligned}
$$

Note all the controlled- $Z$ gates commute with each other, so the order doesn't matter.

Now we generalize the result to $N$-qumode case. We use an adjacency matrix $\mathbf{A}$ to present the whole set of controlled- $Z$ gates rather than enumerating all of them. For example, for Fig. 2.2(c), the adjacency matrix $\mathbf{A}$ is:

$$
\mathbf{A}=\left(\begin{array}{llll}
0 & 1 & 1 & 0 \\
1 & 0 & 0 & 1 \\
1 & 0 & 0 & 1 \\
0 & 1 & 1 & 0
\end{array}\right)
$$

We can use $\hat{C}_{z}[\mathbf{A}]$ to represent all the controlled- $Z$ gates that will be applied on the initial state $|0\rangle_{p}^{\otimes N}$. With this definition, we can calculate the final state in general 
case 17):

$$
\begin{aligned}
\left|\psi_{\mathbf{A}}\right\rangle & =\hat{C}_{z}[\mathbf{A}]|0\rangle_{p}^{\otimes N} \\
& =\prod_{j, k=1}^{N} \exp \left(\frac{i}{2} A_{j k} \hat{q}_{j} \hat{q}_{k}\right)|0\rangle_{p}^{\otimes N} \\
& =\exp \left(\frac{i}{2} \hat{\mathbf{q}}^{\mathrm{T}} \mathbf{A q}\right)|0\rangle_{p}^{\otimes N}
\end{aligned}
$$

where $\hat{\mathbf{q}}=\left(\hat{q}_{1}, \ldots, \hat{q}_{N}\right)^{\mathrm{T}}$.

Same as qubits, using the state vector (as shown above) is hard to visualize the state, we now use operators to represent the state, the same procedure as the stabilizer formalism. Notice that if $\hat{N}|\psi\rangle=0$, then $\hat{S}=\exp (\hat{N})$ stabilizes $|\psi\rangle$. The operator $\hat{N}$ is called the nullifier of state $|\psi\rangle$. Of course we can still use stabilizers for qumodes, but nullifiers for qumodes have some nicer properties as I will show later, so the community adopts nullifiers for qumode graph states.

What are the nullifiers for the state above? The nullifiers for the initial state are very easy to find: $\hat{\mathbf{p}}|0\rangle_{p}^{\otimes N}=\mathbf{0}$, where $\hat{\mathbf{p}}=\left(\hat{p}_{1}, \ldots, \hat{p}_{N}\right)^{\mathrm{T}}$. Now we just need to apply the controlled- $Z$ on the state [17]:

$$
\begin{aligned}
\hat{C}_{z}[\mathbf{A}] \hat{\mathbf{p}}|0\rangle_{p}^{\otimes N} & =\hat{C}_{z}[\mathbf{A}] \hat{\mathbf{p}} \hat{C}_{z}[\mathbf{A}]^{\mathrm{T}} \hat{C}_{z}[\mathbf{A}]|0\rangle_{p}^{\otimes N} \\
& =(\hat{\mathbf{p}}-\mathbf{A} \hat{\mathbf{q}})\left|\psi_{\mathbf{A}}\right\rangle \\
& =\mathbf{0}
\end{aligned}
$$

So the nullifiers are simply $\hat{\mathbf{p}}-\mathbf{A} \hat{\mathbf{q}}$. So this state can then be represented by the graph with adjacency matrix A. Also, given a graph, one can obtain the nullifiers 
easily.

The unitaries and measurements on the qumode graph state have similar effects as those on a qubit graph state. For example, measuring $\hat{q}$ of a node can delete the node from the graph. Interested readers can look at Ref. 17] for a comprehensive explanation.

\subsubsection{Practical qumodes}

We have defined the graph states for qumodes using the direct analogue to qubits, and everything works well so far. However, the state we begin with for each qumode, $|0\rangle_{p}$, has infinite energy and can't be normalized, so it's not physical and can't be generated experimentally. To fix this, we use a finitely squeezed state to approximate $|0\rangle_{p}$.

The (single-mode) squeezed state is created by applying the squeezing operator $\hat{S}(\alpha)=\exp \left(\alpha \hat{a}^{\dagger 2}-\alpha^{*} \hat{a}^{2}\right) / 2$ on the vacuum state $5|0\rangle:$

$$
\begin{gathered}
\hat{S}(\alpha)|0\rangle=\frac{1}{\cosh \alpha} \sum_{n=0}^{\infty}(-\tanh \alpha)^{n} \frac{\sqrt{(2 n) !}}{2^{n} n !}|2 n\rangle \\
\stackrel{\alpha \rightarrow \infty}{\longrightarrow} \sum_{n=0}^{\infty} \frac{(-1)^{n} \sqrt{(2 n) !}}{2^{n} n !}|2 n\rangle
\end{gathered}
$$

\footnotetext{
${ }^{5}$ Note that $|0\rangle$ represents the zero photon number Fock state, not the infinitely squeezed state $|0\rangle_{p}$.
} 
Compare this to $|0\rangle_{p}$ :

$$
\begin{aligned}
|0\rangle_{p} & =\sum_{n=0}^{\infty}|n\rangle\langle n \mid 0\rangle_{p} \\
& =\sum_{n=0}^{\infty}|n\rangle \frac{H_{n}(0)}{\sqrt{2^{n} n !}} \\
& =\sum_{n=0}^{\infty} \frac{(-1)^{n} \sqrt{(2 n) !}}{2^{n} n !}|2 n\rangle
\end{aligned}
$$

where $H_{n}(x)$ is the Hermite polynomial and I have used:

$$
H_{n}(0)= \begin{cases}\frac{n !(-1)^{\frac{n}{2}}}{\left(\frac{n}{2}\right) !}, & \mathrm{n} \text { is even } \\ 0, & \mathrm{n} \text { is odd }\end{cases}
$$

So we can see that at the limit of infinite squeezing $\alpha \rightarrow \infty$, we have: $\hat{S}(\alpha)|0\rangle \rightarrow$ $|0\rangle_{p}$. The squeezed state can be easily generated experimentally (I will present details in Chapter 3), and we can use it to approximate $|0\rangle_{p}$ and create the approximate cluster states.

We use the approximate nullifiers instead of the ideal nullifiers to represent approximate cluster states. That is to find some graph $\mathbf{Z}$ such that when $\alpha \rightarrow \infty$ : (1) $\hat{\mathbf{p}}-\mathbf{Z} \hat{\mathbf{q}} \rightarrow 0$, (2) $\mathbf{Z} \rightarrow \mathbf{A}$. There are many different choices of $\mathbf{Z}$ which satisfy this condition [14, 18], the most useful one (at least in this thesis) is to choose $\mathbf{Z}=i \operatorname{sech} 2 \alpha \mathbf{I}+\tanh 2 \alpha \mathbf{A}[17$. The advantages of this choice of $\mathbf{Z}$ graph are: one can easily build the connection between the approximate graph states and Gaussian pure states, it's easier to calculate the error and noise introduced by the finite squeezing, and it's the state closest to the ideal state given a squeezing level [17]. As I will in- 
troduce in the next Chapter, Gaussian states are the states we create and manipulate in the lab, by using the $\mathbf{Z}$ graph we can track the evolution of the state easily and formulate it in a very compact way. 


\subsubsection{The $\mathcal{H}$ graph}

Now I introduce another graph: the Hamiltonian graph, or the $\mathcal{H}$ graph for short. The $\mathcal{H}$ graph is a graphical way to represent the Hamiltonian. For the Hamiltonian in this form:

$$
\hat{H}(\mathbf{G})=i \hbar \kappa \sum_{i, j} G_{i j} \hat{a}_{i}^{\dagger} \hat{a}_{j}^{\dagger}+\text { H.c. }
$$

Since interactions only involves two fields, so it can be represented by the adjacency matrix $\mathbf{G}$, where the vertices are the modes, the edges indicate the interactions. The adjacency matrix $\mathbf{G}$ forms the $\mathcal{H}$ graph.

The $\mathcal{H}$ graph gives the Hamiltonian of the system, the $\mathbf{A}$ graph describes the structure of a graph state in the ideal case, the $\mathbf{Z}$ graph represents the graph state with finite squeezing. The $\mathcal{H}$ graph is what we got directly from the lab, I will cover more details about this in the next Chapter. The $\mathbf{A}$ graph and $\mathbf{Z}$ graph represent the final states, so we'd like to know the connection between those three kinds of graphs. In the next section, I will present how to derive the $\mathbf{A}$ graph and $\mathbf{Z}$ graph from the $\mathcal{H}$ graph.

\subsection{From $\mathcal{H}$ graph to $\mathrm{Z}$ graph}

Given an arbitrary $\mathcal{H}$ graph, we'd like to know what the correspond $\mathbf{Z}$ graph is. I will derive it in both Heisenberg and Schrödinger picture. 


\subsubsection{Heisenberg picture}

The general Hamiltonian of Eq. 2.36 leads to temporal evolution $\hat{U}=\exp \left(-\frac{i}{\hbar} \hat{H}(\mathbf{G}) t\right)$ from the initial vacuum state $|0\rangle$ in the Schrödinger picture, and applies $\hat{U}^{\dagger} \hat{a} \hat{U}$ to the field $\hat{a}$ in the Heisenberg picture. We first investigate the Heisenberg picture.

Let's first look at the Hamiltonian of only two qumodes: $\hat{H}=2 i \hbar \kappa \hat{a}_{1}^{\dagger} \hat{a}_{2}^{\dagger}+H . c$. . Comparing with Eq. (2.36), we have:

$$
\mathbf{G}=\left(\begin{array}{ll}
0 & 1 \\
1 & 0
\end{array}\right)
$$

Using the Heisenberg equations, we have:

$$
\begin{aligned}
\frac{d \hat{a}_{1}}{d t} & =\frac{1}{i \hbar}\left[\hat{a}_{1}, \hat{H}\right] \\
& =\kappa\left[\hat{a}_{1}, 2 \hat{a}_{1}^{\dagger} \hat{a}_{2}^{\dagger}\right] \\
& =2 \kappa \hat{a}_{2} \\
\frac{d \hat{a}_{2}}{d t} & =2 \kappa \hat{a}_{1}
\end{aligned}
$$

To write it in a more compact way, let: $\hat{\mathbf{a}}=\left(\hat{a}_{1}, \hat{a}_{2}\right)^{\mathrm{T}}$. Then the above result can be written as:

$$
\frac{d \hat{\mathbf{a}}}{d t}=2 \kappa \mathbf{G} \hat{\mathbf{a}}^{\dagger}
$$


Solve this equation, we have

$$
\hat{\mathbf{a}}(r)=e^{r \mathbf{G}} \hat{\mathbf{a}}(0)
$$

where $r=2 \kappa t>0$ is an overall squeezing parameter. So the quadratures satisfy:

$$
\left(\begin{array}{c}
\hat{\mathbf{q}}(r) \\
\hat{\mathbf{p}}(r)
\end{array}\right)=\left(\begin{array}{cc}
e^{r \mathbf{G}} & \mathbf{0} \\
\mathbf{0} & e^{-r \mathbf{G}}
\end{array}\right)\left(\begin{array}{c}
\hat{\mathbf{q}}(0) \\
\hat{\mathbf{p}}(0)
\end{array}\right)
$$

where $\hat{\mathbf{q}}=\left(\hat{q}_{1}, \hat{q}_{2}\right)^{\mathrm{T}}, \hat{\mathbf{p}}=\left(\hat{p}_{1}, \hat{p}_{2}\right)^{\mathrm{T}}$. This result can be generalized to $N$ qumode case, the solution is in the same form as the above equation.

Now we apply a balanced beamsplitter to the field out from the OPO (this step is very useful, I will talk about the details later.). In the Heisenberg picture, a balanced beamsplitter can be represented by a unitary matrix $\mathbf{R}$ in which all entries have equal magnitude. In the two-qumode case, we have:

$$
\mathbf{R}=\frac{1}{\sqrt{2}}\left(\begin{array}{cc}
1 & 1 \\
1 & -1
\end{array}\right)
$$

The transformation on quadratures is:

$$
\left(\begin{array}{c}
\hat{\mathbf{q}}(r) \\
\hat{\mathbf{p}}(r)
\end{array}\right)=\left(\begin{array}{cc}
\mathbf{R} e^{r \mathbf{G}} & \mathbf{0} \\
\mathbf{0} & \mathbf{R} e^{-r \mathbf{G}}
\end{array}\right)\left(\begin{array}{c}
\hat{\mathbf{q}}(0) \\
\hat{\mathbf{p}}(0)
\end{array}\right)
$$

In state verification and other applications (we will discuss later), we need to measure the squeezing level, which involves measurements of quadrature variances.

\footnotetext{
${ }^{6}$ Note: from now on, to differentiate between the two pictures, the operators without an argument (e.g., $\hat{\mathbf{q}}$ ) are in the Schrödinger picture (which means they don't evolve), the operators with an argument (e.g., $\hat{\mathbf{q}}(r), \hat{\mathbf{q}}(0))$ are in the Heisenberg picture.
} 
So it's useful to calculate the covariance of the quadratures.

For two Hermitian operators $\hat{x}_{1}$ and $\hat{x}_{2}$, the covariance is defined as :

$$
\operatorname{cov}\left(\hat{x}_{1}, \hat{x}_{2}\right)=\frac{1}{2}\left\langle\hat{x}_{1} \hat{x}_{2}+\hat{x}_{2} \hat{x}_{1}\right\rangle-\left\langle\hat{x}_{1}\right\rangle\left\langle\hat{x}_{2}\right\rangle
$$

In this Chapter, I set $\langle\hat{x}\rangle=0$ for all operator $\hat{x}$, since I'm focused on quadrature operators with zero mean. The non-zero mean case can be handled by applying displacement operations [17].

The covariance matrix for $\hat{\mathbf{x}}=\left(\hat{x}_{1}, \hat{x}_{2}\right)^{\mathrm{T}}$ is defined as:

$$
\begin{aligned}
\operatorname{cov}(\hat{\mathbf{x}}) & :=\left(\begin{array}{cc}
\operatorname{cov}\left(\hat{x}_{1}, \hat{x}_{1}\right) & \operatorname{cov}\left(\hat{x}_{1}, \hat{x}_{2}\right) \\
\operatorname{cov}\left(\hat{x}_{1}, \hat{x}_{2}\right) & \operatorname{cov}\left(\hat{x}_{2}, \hat{x}_{2}\right)
\end{array}\right) \\
& =\left\langle\psi\left|\left(\begin{array}{cc}
\hat{x}_{1} \hat{x}_{1} & \hat{x}_{1} \hat{x}_{2} \\
\hat{x}_{2} \hat{x}_{1} & \hat{x}_{2} \hat{x}_{2}
\end{array}\right)\right| \psi\right\rangle \\
& =\left\langle\psi\left|\hat{\mathbf{x}} \hat{\mathbf{x}}^{\mathrm{T}}\right| \psi\right\rangle
\end{aligned}
$$

When $\hat{\mathbf{x}}=\left(\hat{\mathbf{q}}^{\mathrm{T}}, \hat{\mathbf{p}}^{\mathrm{T}}\right)^{\mathrm{T}}$, we have:

$$
\begin{aligned}
\operatorname{cov}\left(\begin{array}{c}
\hat{\mathbf{q}} \\
\hat{\mathbf{p}}
\end{array}\right) & =\left\langle\psi\left|\left(\begin{array}{cc}
\hat{\mathbf{q}} \hat{\mathbf{q}}^{\mathrm{T}} & \hat{\mathbf{q}} \hat{\mathbf{p}}^{\mathrm{T}} \\
\hat{\mathbf{p}} \hat{\mathbf{q}}^{\mathrm{T}} & \hat{\mathbf{p}} \hat{\mathbf{p}}^{\mathrm{T}}
\end{array}\right)\right| \psi\right\rangle \\
& =\left\langle 0\left|\left(\begin{array}{cc}
\hat{\mathbf{q}}(r) \hat{\mathbf{q}}(r)^{\mathrm{T}} & \hat{\mathbf{q}}(r) \hat{\mathbf{p}}(r)^{\mathrm{T}} \\
\hat{\mathbf{p}}(r) \hat{\mathbf{q}}(r)^{\mathrm{T}} & \hat{\mathbf{p}}(r) \hat{\mathbf{p}}(r)^{\mathrm{T}}
\end{array}\right)\right| 0\right\rangle \\
& =\frac{1}{2}\left(\begin{array}{cc}
\mathbf{R} e^{2 r G} \mathbf{R}^{\mathrm{T}} & \mathbf{0} \\
\mathbf{0} & \mathbf{R} e^{-2 r \mathbf{G}} \mathbf{R}^{\mathrm{T}}
\end{array}\right)
\end{aligned}
$$


where I have used Eq. (2.43) and:

$$
\begin{aligned}
& \langle\hat{q}(0) \hat{q}(0)\rangle=\langle\hat{p}(0) \hat{p}(0)\rangle=\frac{1}{2} \\
& \langle\hat{q}(0) \hat{p}(0)\rangle=\langle\hat{p}(0) \hat{q}(0)\rangle=0
\end{aligned}
$$

This result holds for $N$-qumode cases as well.

If $\mathbf{G}$ is self-inverse, then:

$$
\begin{aligned}
e^{2 r \mathbf{G}} & =\sum_{n=0}^{\infty} \frac{(2 r \mathbf{G})^{n}}{n !} \\
& =c \mathbf{I}+s \mathbf{G}
\end{aligned}
$$

where $c=\cosh 2 r, s=\sinh 2 r$. So we have:

$$
\operatorname{cov}\left(\begin{array}{c}
\hat{\mathbf{q}} \\
\hat{\mathbf{p}}
\end{array}\right)=\frac{1}{2}\left(\begin{array}{cc}
c \mathbf{I}+s \mathbf{R} \mathbf{G} \mathbf{R}^{\mathrm{T}} & \mathbf{0} \\
\mathbf{0} & c \mathbf{I}-s \mathbf{R G R}^{\mathrm{T}}
\end{array}\right)
$$

With Eq. 2.43), we can calculate the covariance of all linear combinations of quadratures.

Now we derive the nullifiers of the final state. We first look at the nullifiers before BS (right after OPO), where the Hamiltonian is written in Eq. 2.36). We know the state here is a collection of two-mode squeezed states. If mode $i$ and mode $j$ are entangled, the nullifier for this pair is:

$$
\hat{q}_{i, \theta}\left(t_{1}\right)-\hat{q}_{j,-\theta}\left(t_{1}\right) \rightarrow 0
$$


where $\hat{q}_{i, \theta}=\hat{q}_{i} \cos \theta+\hat{p}_{i} \sin \theta$. Whether mode $i$ and mode $j$ are entangled depends on whether $G_{i j}$ in $\mathbf{G}$ is nonzero. So taking the whole Hamiltonian into consideration, the nullifier vector is:

$$
\hat{\mathbf{q}}_{\theta}\left(t_{1}\right)-\mathbf{G} \hat{\mathbf{q}}_{-\theta}\left(t_{1}\right) \rightarrow 0
$$

After the OPO, the BS transforms $\hat{\mathbf{q}}_{\theta}\left(t_{1}\right)$ into $\hat{\mathbf{q}}_{\theta}\left(t_{2}\right)=\mathbf{R} \hat{\mathbf{q}}_{\theta}\left(t_{1}\right)$, so the final nullifier vector in the Heisenberg picture is:

$$
\mathbf{R}^{\mathrm{T}} \hat{\mathbf{q}}_{\theta}\left(t_{2}\right)-\mathbf{G R}^{\mathrm{T}} \hat{\mathbf{q}}_{-\theta}\left(t_{2}\right) \rightarrow 0
$$

Now we check the covariance of the nullifier vector $\mathbf{R}^{\mathrm{T}} \hat{\mathbf{q}}_{\theta}-\mathbf{G R}^{\mathrm{T}} \hat{\mathbf{q}}_{-\theta}$ :

$$
\begin{aligned}
\operatorname{cov}\left(\mathbf{R}^{\mathrm{T}} \hat{\mathbf{q}}_{\theta}-\mathbf{G} \mathbf{R}^{\mathrm{T}} \hat{\mathbf{q}}_{-\theta}\right)= & \langle\psi|\left(\mathbf{R}^{\mathrm{T}} \hat{\mathbf{q}}_{\theta} \hat{\mathbf{q}}_{\theta}^{\mathrm{T}} \mathbf{R}+\mathbf{G} \mathbf{R}^{\mathrm{T}} \hat{\mathbf{q}}_{\theta} \hat{\mathbf{q}}_{\theta}^{\mathrm{T}} \mathbf{R} \mathbf{G}^{\mathrm{T}}\right. \\
& \left.-\mathbf{G} \mathbf{R}^{\mathrm{T}} \hat{\mathbf{q}}_{-\theta} \hat{\mathbf{q}}_{\theta}^{\mathrm{T}} \mathbf{R}-\mathbf{R}^{\mathrm{T}} \hat{\mathbf{q}}_{\theta} \hat{\mathbf{q}}_{-\theta}^{\mathrm{T}} \mathbf{R G}^{\mathrm{T}}\right)|\psi\rangle \\
= & e^{-2 r} \mathbf{I}
\end{aligned}
$$

We can see the variances (diagonal terms in covariance matrix) go to zero as $r \rightarrow \infty$, indicating this is the nullifier vector of the final state [19].

By applying local transformations and rearranging the nullifiers into the form of $\hat{\mathbf{p}}-\mathbf{A} \hat{\mathbf{q}}$, one can find out the corresponding $\mathbf{A}$ graph, which is the adjacency matrix for the graph state at infinitely squeezing limit.

\subsubsection{Schrödinger picture}

We now solve the same problem in a different picture: the Schrödinger picture. Ref. [17] proposed a $\mathbf{Z}$ graph which can be used to represent any zero-mean Gaussian 
pure state. The complex matrix $\mathbf{Z}$ can be written as: $\mathbf{Z}=\mathbf{V}+i \mathbf{U}$, where $\mathbf{V}$ and $\mathbf{U}$ are real matrices. If $\mathbf{U} \rightarrow \mathbf{0}$ at infinite squeezing limit, the $\mathbf{Z}$ graph becomes the $\mathbf{A}$ graph: $\mathbf{V} \rightarrow \mathbf{A}$.

For a given $|\psi\rangle, \mathbf{Z}$ can be obtained from the covariance matrix of quadratures. By comparing the covariance matrix in Eq. (2.46) with Eq. (2.19) in Ref. [17], we find:

$$
\begin{aligned}
\mathbf{Z} & =\mathbf{V}+i \mathbf{U} \\
& =i \mathbf{R} e^{-2 r \mathbf{G}} \mathbf{R}^{\mathrm{T}}
\end{aligned}
$$

For any $\mathbf{Z}$ graph, this relation holds: $(\hat{\mathbf{p}}-\mathbf{Z} \hat{\mathbf{q}})|\psi\rangle=\mathbf{0} 17]$. In our case:

$$
\left(\hat{\mathbf{p}}-i \mathbf{R} e^{-2 r \mathbf{G}} \mathbf{R}^{\mathrm{T}} \hat{\mathbf{q}}\right)|\psi\rangle=\mathbf{0}
$$

Note in this case when $r \rightarrow \infty$, the imaginary part of $\mathbf{Z}$ ( $\mathbf{Z}$ is pure imaginary in this case) doesn't go to zero, so it's not clear what the corresponding $\mathbf{A}$ is at this point. Before finding the $\mathbf{A}$ graph, we first look at the nullifiers of the final state.

In the previous section, we derived in Heisenberg picture that the nullifier vector is $\mathbf{R}^{\mathrm{T}} \hat{\mathbf{q}}_{\theta}-\mathbf{G R}^{\mathrm{T}} \hat{\mathbf{q}}_{-\theta}$, now how do we derive the nullifiers using the above relation, in the Schrödinger picture?

\footnotetext{
${ }^{7}$ Note $(\hat{\mathbf{p}}-\mathbf{A} \hat{\mathbf{q}})|\psi\rangle$ goes to zero at infinite squeezing limit, but $(\hat{\mathbf{p}}-\mathbf{Z} \hat{\mathbf{q}})|\psi\rangle$ equals to zero for all squeezing levels.
} 
We first separate the real and imaginary part by left-multiply $-i \mathbf{R}^{\mathrm{T}}$ and $-i e^{2 r \mathbf{G}}$ :

$$
\begin{aligned}
e^{-2 r \mathbf{G}} \mathbf{R}^{\mathrm{T}} \hat{\mathbf{q}}|\psi\rangle & =-i \mathbf{R}^{\mathrm{T}} \hat{\mathbf{p}}|\psi\rangle \\
e^{2 r \mathbf{G}} \mathbf{R}^{\mathrm{T}} \hat{\mathbf{p}}|\psi\rangle & =i \mathbf{R}^{\mathrm{T}} \hat{\mathbf{q}}|\psi\rangle
\end{aligned}
$$

If $\mathbf{G}$ is self-inverse, $\mathbf{G}^{2}=\mathbf{I}$, then Eq. (2.56) becomes:

$$
\begin{aligned}
& (\mathbf{I}-t \mathbf{G}) \mathbf{R}^{\mathrm{T}} \hat{\mathbf{q}}|\psi\rangle=-i \epsilon \mathbf{R}^{\mathrm{T}} \hat{\mathbf{p}}|\psi\rangle \\
& (\mathbf{I}+t \mathbf{G}) \mathbf{R}^{\mathrm{T}} \hat{\mathbf{p}}|\psi\rangle=i \epsilon \mathbf{R}^{\mathrm{T}} \hat{\mathbf{q}}|\psi\rangle
\end{aligned}
$$

where $t=\tanh 2 r, \epsilon=\operatorname{sech} 2 r$. Since $\epsilon \stackrel{r \rightarrow+\infty}{\longrightarrow} 0$, so the right-hand side goes to zero at infinite squeezing limit. Combine these two equations:

$$
\left[\mathbf{R}^{\mathrm{T}} \hat{\mathbf{q}}_{\theta}-t \mathbf{G R}^{\mathrm{T}} \hat{\mathbf{q}}_{-\theta}\right]|\psi\rangle=-i \epsilon \mathbf{R}^{\mathrm{T}} \hat{\mathbf{q}}_{\theta+\frac{\pi}{2}}|\psi\rangle \stackrel{r \rightarrow+\infty}{\longrightarrow} \mathbf{0}
$$

Clearly, $\mathbf{R}^{\mathrm{T}} \hat{\mathbf{q}}_{\theta}-t \mathbf{G} \mathbf{R}^{\mathrm{T}} \hat{\mathbf{q}}_{-\theta}$ is a nullifier vector. Compare Eq. 2.53 with Eq. 2.58, we can see the latter has an additional coefficient $t$. As I pointed out earlier, there are infinite choices of approximate nullifiers. For this particular case, a particular set of nullifiers is to replace the coefficient $t$ by $t^{\prime}$, as long as $t^{\prime} \rightarrow 1$ when $r \rightarrow+\infty$, we will have $\left[\mathbf{R}^{\mathrm{T}} \hat{\mathbf{q}}_{\theta}-t^{\prime} \mathbf{G R}^{\mathrm{T}} \hat{\mathbf{q}}_{-\theta}\right]|\psi\rangle \stackrel{r \rightarrow+\infty}{\longrightarrow} \mathbf{0}$.

To deviate from the main topic (that is to find $\mathbf{Z}$ and nullifiers given $\mathcal{H}$ graph, which has been done for the self-inverse $\mathbf{G}$ case) a little bit, it is interesting to find what $t^{\prime}$ minimized the variance of the approximate nullifiers $\mathbf{R}^{\mathrm{T}} \hat{\mathbf{q}}_{\theta}-t^{\prime} \mathbf{G} \mathbf{R}^{\mathrm{T}} \hat{\mathbf{q}}_{-\theta}$, for a certain squeezing parameter $r$. 
With Eq. (2.46), we got:

$$
\begin{aligned}
& \operatorname{cov}\left(\mathbf{R}^{\mathrm{T}} \hat{\mathbf{q}}_{\theta}-t^{\prime} \mathbf{G} \mathbf{R}^{\mathrm{T}} \hat{\mathbf{q}}_{-\theta}\right) \\
= & \frac{1}{2}\left(\left[c\left(t^{\prime}-t\right)^{2}+\epsilon\right] \mathbf{I}+\left[s\left(t^{\prime}-t\right)^{2}-\delta\right] \mathbf{G} \cos 2 \theta\right)
\end{aligned}
$$

where $\delta=\operatorname{csch} 2 r$.

The variances are the diagonal terms in the covariance matrix. We can clearly see that the minimum variance is achieved when $t^{\prime}=t$, which corresponds to the nullifiers in Eq. 2.53). So the nullifiers derived in the Schrödinger picture using the $\mathbf{Z}$ graph achieve the minimum variance. In fact, Ref. [17] proved that the $\mathbf{Z}$ graph is the "closest" cluster state to the ideal cluster state, which means the approximate nullifiers of this state have the minimum variance over other choices of approximate cluster states.

However, smallest variance doesn't mean largest squeezing, because the squeezing level is a ratio between the variance of final state and the variance of the vacuum. When the coefficient changes, both the variance of the final state and the variance of the corresponding vacuum will change.

The covariance of the vacuum is:

$$
\left\langle 0\left|\left(\mathbf{R}^{\mathrm{T}} \hat{\mathbf{q}}_{\theta}-t^{\prime} \mathbf{G} \mathbf{R}^{\mathrm{T}} \hat{\mathbf{q}}_{-\theta}\right)\left(\mathbf{R}^{\mathrm{T}} \hat{\mathbf{q}}_{\theta}-t^{\prime} \mathbf{G} \mathbf{R}^{\mathrm{T}} \hat{\mathbf{q}}_{-\theta}\right)^{\mathrm{T}}\right| 0\right\rangle=\frac{1}{2}\left(1+t^{\prime 2}\right) \mathbf{I}
$$

It clearly depends on the $t^{\prime}$. Now the diagonal terms in the covariance matrix, 
which are the variances, become:

$$
\frac{c\left(t^{\prime}-t\right)^{2}+\epsilon}{1+t^{\prime 2}}=c+\frac{2 s}{t^{\prime}+\frac{1}{t^{\prime}}}
$$

This is minimized when $t^{\prime}=1$, with a minimum value of $e^{-2 r}$. So although the nullifiers in Eq. (2.58) $\left(t^{\prime}=t\right)$ have the smallest variances, the largest squeezing is achieved by the nullifiers in Eq. (2.53) $\left(t^{\prime}=1\right)$.

As we will point out later, in the state verification experiment, the coefficient $t^{\prime}$ is actually the ratio between two reference fields (known as local oscillators) used for quadrature measurements. By controlling the powers of the two fields, we can choose any coefficient $t^{\prime}$ for different approximate nullifiers.

Now we come back to the main topic. So far I have covered the cases when $\mathbf{G}$ is self-inverse. In the following Section, I provide a method for general G.

\subsubsection{When $\mathrm{G}$ is not self-inverse}

Ref. [17] is focused on self-inverse $\mathbf{G}$, which provides an easy connection to $\mathbf{Z}$ and A graph. Ref. 20] studied a 4-qumode non-self-inverse G, but didn't generalize the method. In here, I present a method for finding the nullifiers and $\mathbf{Z}$ graph for any bipartite $\mathbf{G}$.

In Eq. 2.56), $\mathrm{G}$ is on the exponent, which makes it hard for linear transformations. When $\mathbf{G}$ is self-inverse, the equation becomes linear in $\mathbf{G}$, which significantly simplifies the equation. When $\mathbf{G}$ is not self-inverse, we can diagonalize $\mathbf{G}$ to for calculating the exponential term. 
Let $\left(\mathbf{c}_{1}, \ldots, \mathbf{c}_{N}\right)$ be $\mathbf{G}$ 's eigenvectors: $\mathbf{G c}_{i}=\lambda_{i} \mathbf{c}_{i}$, and $\mathbf{C}=\left(\mathbf{c}_{1}, \ldots, \mathbf{c}_{N}\right)$, we have:

$$
\begin{gathered}
\mathbf{C}^{\mathrm{T}} \mathbf{G C}=\bigoplus_{i=1}^{N} \lambda_{i} \\
\mathbf{G}=\sum_{i=1}^{N} \lambda_{i} \mathbf{c}_{i} \mathbf{c}_{i}^{\mathrm{T}}
\end{gathered}
$$

The exponential term can then be written as:

$$
\begin{aligned}
& \mathbf{C}^{\mathrm{T}} e^{r \mathbf{G}} \mathbf{C}=\bigoplus_{i=1}^{N} e^{r \lambda_{i}} \\
& e^{r \mathbf{G}}=\sum_{i=1}^{N} e^{r \lambda_{i}} \mathbf{c}_{i} \mathbf{c}_{i}^{\mathrm{T}}
\end{aligned}
$$

We first look at the special case that $\mathbf{G}$ 's dimension is $N=2 n$, and its eigenvalues are paired: $\left\{ \pm \lambda_{i}, \lambda_{i}>0, i \in[1, n]\right\}$. Let $\mathbf{G c}_{i}=-\lambda_{i} \mathbf{c}_{i}, \mathbf{G c}_{i+n}=\lambda_{i} \mathbf{c}_{i+n}, i \in[1, n]$. With this assumption, $\mathbf{G}$ can be diagonalized as:

$$
\mathbf{C}^{\mathrm{T}} \mathbf{G C}=\left(\begin{array}{cc}
\bigoplus_{i=1}^{n}-\lambda_{i} & \mathbf{0} \\
\mathbf{0} & \bigoplus_{i=1}^{n} \lambda_{i}
\end{array}\right)
$$

We can expand the exponential term of $\mathbf{G}$ in Eq. 2.56 using $\mathbf{G}$ 's spectrum:

$$
\begin{aligned}
& \left(\sum_{i=1}^{n} e^{-2 r \lambda_{i}} \mathbf{c}_{i} \mathbf{c}_{i}^{\mathrm{T}}\right) \mathbf{R}^{\mathrm{T}} \hat{\mathbf{q}}|\psi\rangle=-i \mathbf{R}^{\mathrm{T}} \hat{\mathbf{p}}|\psi\rangle, i \in[1, n] \\
& \left(\sum_{i=1}^{n} e^{2 r \lambda_{i}} \mathbf{c}_{i} \mathbf{c}_{i}^{\mathrm{T}}\right) \mathbf{R}^{\mathrm{T}} \hat{\mathbf{p}}|\psi\rangle=i \mathbf{R}^{\mathrm{T}} \hat{\mathbf{q}}|\psi\rangle, i \in[n+1,2 n]
\end{aligned}
$$


Using the orthogonality: $\mathbf{c}_{i}^{\mathrm{T}} \mathbf{c}_{j}=\delta_{i j}$, we can get:

$$
\begin{aligned}
\mathbf{c}_{i}^{\mathrm{T}} \mathbf{R}^{\mathrm{T}} \hat{\mathbf{q}}|\psi\rangle & =-i e^{2 r \lambda_{i}} \mathbf{c}_{i}^{\mathrm{T}} \mathbf{R}^{\mathrm{T}} \hat{\mathbf{p}}|\psi\rangle \stackrel{\lambda_{i}<0, r \rightarrow+\infty}{\longrightarrow} \mathbf{0} \\
\mathbf{c}_{i}^{\mathrm{T}} \mathbf{R}^{\mathrm{T}} \hat{\mathbf{p}}|\psi\rangle & =i e^{-2 r \lambda_{i}} \mathbf{c}_{i}^{\mathrm{T}} \mathbf{R}^{\mathrm{T}} \hat{\mathbf{q}}|\psi\rangle \stackrel{\lambda_{i}>0, r \rightarrow+\infty}{\longrightarrow} \mathbf{0}
\end{aligned}
$$

We have found the nullifiers. Let $\boldsymbol{\epsilon}=\left(\bigoplus_{j=1}^{n} e^{-2 r \lambda_{j}}\right)$, then the nullifiers can also be written as:

$$
\begin{aligned}
& \left(\begin{array}{cc}
\mathbf{I}_{n} & \mathbf{0} \\
\mathbf{0} & \mathbf{0}
\end{array}\right) \mathbf{C}^{\mathrm{T}} \mathbf{R}^{\mathrm{T}} \hat{\mathbf{q}}|\psi\rangle=-i\left(\begin{array}{cc}
\boldsymbol{\epsilon} & \mathbf{0} \\
\mathbf{0} & \mathbf{0}
\end{array}\right) \mathbf{C}^{\mathrm{T}} \mathbf{R}^{\mathrm{T}} \hat{\mathbf{p}}|\psi\rangle \stackrel{r \rightarrow+\infty}{\longrightarrow} \mathbf{0} \\
& \left(\begin{array}{cc}
\mathbf{0} & \mathbf{0} \\
\mathbf{I}_{n} & \mathbf{0}
\end{array}\right) \mathbf{C}^{\mathrm{T}} \mathbf{R}^{\mathrm{T}} \hat{\mathbf{p}}|\psi\rangle=i\left(\begin{array}{cc}
\mathbf{0} & \mathbf{0} \\
\boldsymbol{\epsilon} & \mathbf{0}
\end{array}\right) \mathbf{C}^{\mathrm{T}} \mathbf{R}^{\mathrm{T}} \hat{\mathbf{q}}|\psi\rangle \stackrel{r \rightarrow+\infty}{\longrightarrow} \mathbf{0}
\end{aligned}
$$

The nullifiers tell us what quadrature combinations to measure in state verification, but we are also interested in what the graph is for this state. We now derive the $\mathbf{Z}$ graph for a general $\mathbf{G}$.

$$
\begin{aligned}
& \text { Let } \mathbf{C}^{\mathrm{T}}=\left(\begin{array}{cc}
\mathbf{C}_{1} & \mathbf{C}_{2} \\
\mathbf{C}_{3} & \mathbf{C}_{4}
\end{array}\right), \hat{\mathbf{Q}}=\mathbf{R}^{\mathrm{T}} \hat{\mathbf{q}}=\left(\begin{array}{c}
\hat{\mathbf{Q}}_{1} \\
\hat{\mathbf{Q}}_{2}
\end{array}\right), \hat{\mathbf{P}}=\mathbf{R}^{\mathrm{T}} \hat{\mathbf{p}}=\left(\begin{array}{c}
\hat{\mathbf{P}}_{1} \\
\hat{\mathbf{P}}_{2}
\end{array}\right) \text {, and } \\
& \boldsymbol{\epsilon}=\left(\bigoplus_{j=1}^{n} e^{-2 r \lambda_{j}}\right) \text {, then Eq. 2.67) can be written as: }
\end{aligned}
$$




$$
\begin{aligned}
& \left(\begin{array}{cc}
\mathbf{C}_{1} & \mathbf{C}_{2} \\
\mathbf{0} & 0
\end{array}\right) \hat{\mathbf{Q}}|\psi\rangle=-i\left(\begin{array}{cc}
\boldsymbol{\epsilon} \mathbf{C}_{1} & \boldsymbol{\epsilon} \mathbf{C}_{2} \\
0 & 0
\end{array}\right) \hat{\mathbf{P}}|\psi\rangle \\
& \left(\begin{array}{cc}
0 & 0 \\
\mathbf{C}_{3} & \mathbf{C}_{4}
\end{array}\right) \hat{\mathbf{P}}|\psi\rangle=i\left(\begin{array}{cc}
0 & 0 \\
\epsilon \mathbf{C}_{3} & \boldsymbol{\epsilon} \mathbf{C}_{4}
\end{array}\right) \hat{\mathbf{Q}}|\psi\rangle
\end{aligned}
$$

Put the two equations together:

$$
\left(\begin{array}{cc}
\mathbf{C}_{1} & \mathbf{C}_{2} \\
-i \epsilon \mathbf{C}_{3} & -i \boldsymbol{\epsilon} \mathbf{C}_{4}
\end{array}\right) \hat{\mathbf{Q}}|\psi\rangle+\left(\begin{array}{cc}
i \boldsymbol{\epsilon} \mathbf{C}_{1} & i \boldsymbol{\epsilon} \mathbf{C}_{2} \\
\mathbf{C}_{3} & \mathbf{C}_{4}
\end{array}\right) \hat{\mathbf{P}}|\psi\rangle=\mathbf{0}
$$

Now apply the Fourier transformation on the first half of modes: $\hat{\mathbf{Q}}_{1} \rightarrow \hat{\mathbf{P}}_{1}$, $\hat{\mathbf{P}}_{1} \rightarrow-\hat{\mathbf{Q}}_{1}$, then

$$
\left(\begin{array}{cc}
\mathbf{C}_{1} & i \boldsymbol{\epsilon} \mathbf{C}_{2} \\
-i \boldsymbol{\epsilon} \mathbf{C}_{3} & \mathbf{C}_{4}
\end{array}\right) \hat{\mathbf{P}}|\psi\rangle-\left(\begin{array}{cc}
i \boldsymbol{\epsilon} \mathbf{C}_{1} & -\mathbf{C}_{2} \\
\mathbf{C}_{3} & i \boldsymbol{\epsilon} \mathbf{C}_{4}
\end{array}\right) \hat{\mathbf{Q}}|\psi\rangle=\mathbf{0}
$$

If the matrix before $\hat{\mathbf{P}}$ is invertible, then:

$$
\left[\hat{\mathbf{p}}-\mathbf{R}\left(\begin{array}{cc}
\mathbf{C}_{1} & i \boldsymbol{\epsilon} \mathbf{C}_{2} \\
-i \epsilon \mathbf{C}_{3} & \mathbf{C}_{4}
\end{array}\right)^{-1}\left(\begin{array}{cc}
i \boldsymbol{\epsilon} \mathbf{C}_{1} & -\mathbf{C}_{2} \\
\mathbf{C}_{3} & i \epsilon \mathbf{C}_{4}
\end{array}\right) \mathbf{R}^{\mathrm{T}} \hat{\mathbf{q}}\right]|\psi\rangle=\mathbf{0}
$$

Comparing with $\hat{\mathbf{p}}-\mathbf{Z} \hat{\mathbf{q}}=\mathbf{0}$, we have:

$$
\mathbf{Z}=\mathbf{R}\left(\begin{array}{cc}
\mathbf{C}_{1} & i \epsilon \mathbf{C}_{2} \\
-i \epsilon \mathbf{C}_{3} & \mathbf{C}_{4}
\end{array}\right)^{-1}\left(\begin{array}{cc}
i \epsilon \mathbf{C}_{1} & -\mathbf{C}_{2} \\
\mathbf{C}_{3} & i \epsilon \mathbf{C}_{4}
\end{array}\right) \mathbf{R}^{\mathrm{T}}
$$

Now we apply this result to two special cases. 


\subsubsection{The Square Cluster State}

First, we look at the case in Ref. [20], where the $\mathcal{H}$ graph is:

$$
\mathbf{G}=\left(\begin{array}{cccc}
0 & 1 & 0 & 0 \\
1 & 0 & 1 & 0 \\
0 & 1 & 0 & 1 \\
0 & 0 & 1 & 0
\end{array}\right)
$$

The eigenvalues for this matrix are: $\left\{-\Phi,-\frac{1}{\Phi}, \Phi, \frac{1}{\Phi}\right\}$, where $\Phi=\frac{1+\sqrt{5}}{2}$. In this case, the matrix $\mathbf{C}$ formed by its eigenvectors is:

$$
\mathbf{C}=\frac{1}{\sqrt{5+\sqrt{5}}}\left(\begin{array}{cccc}
-1 & \Phi & 1 & -\Phi \\
\Phi & -1 & \Phi & -1 \\
-\Phi & -1 & \Phi & 1 \\
1 & \Phi & 1 & \Phi
\end{array}\right)
$$

Using Eq. (2.67), we can easily find all the nullifiers, which are consistent with Eq. (2-5) in Ref. [20], with qumode 1 here corresponds to qumode $(-n, y)$ in Ref. [20], qumode 2 to qumode $(n, y)$, qumode 3 to qumode $(-n, z)$, and qumode 4 to qumode $(n, z)$.

Now we check what the $\mathbf{A}$ is. Using Eq. 2.72), and let $\boldsymbol{\epsilon} \rightarrow 0$, we get the $\mathbf{A}$ 
graph:

$$
A=\left(\begin{array}{cccc}
0 & 0 & 2 & -\sqrt{5} \\
0 & 0 & \sqrt{5} & -2 \\
2 & \sqrt{5} & 0 & 0 \\
-\sqrt{5} & -2 & 0 & 0
\end{array}\right)
$$

This is a square graph state and is consistent with the result in Ref. [20]. Note in here the Fourier transformation is applied on the first two qumodes, applying it on different qumodes can lead to a $\mathbf{A}$ graph of different weights, but those graphs are LU-equivalent.

\subsubsection{The Self-inverse G}

Now we check whether our result is consistent with Ref. [17 where the self-inverse cases are discussed. When $\mathbf{G}$ is bipartite and self-inverse, it can be written in the form as Eq. (4.15) in Ref. 17]:

$$
\mathbf{G}=\left(\begin{array}{cc}
\mathbf{0} & \mathbf{G}_{0}^{\mathrm{T}} \\
\mathbf{G}_{0} & \mathbf{0}
\end{array}\right)
$$

where $\mathbf{G}_{0}$ has a dimension of $n=N / 2$. In this case, we have:

$$
\begin{aligned}
& \mathbf{C}^{\mathrm{T}}=\frac{1}{\sqrt{2}}\left(\begin{array}{cc}
-\mathbf{I} & \mathbf{G}_{0} \\
\mathbf{G}_{0} & \mathbf{I}
\end{array}\right) \\
& \mathbf{C}^{\mathrm{T}} \mathbf{G C}=\left(\begin{array}{cc}
-\mathbf{I} & \mathbf{0} \\
\mathbf{0} & \mathbf{I}
\end{array}\right)
\end{aligned}
$$


With this, Eq. 2.72 becomes:

$$
\mathbf{Z}=\mathbf{R}\left(\begin{array}{cc}
i \operatorname{sech} 2 r \mathbf{I} & \tanh 2 r \mathbf{G}_{0} \\
\tanh 2 r \mathbf{G}_{0} & i \operatorname{sech} 2 r \mathbf{I}
\end{array}\right) \mathbf{R}^{\mathrm{T}}
$$

this gives the same result as Eq. (4.17) in Ref. [17] (in Ref. [17] $\mathbf{R}=\mathbf{I}$ ).

\subsubsection{More General Cases}

What if G's eigenvalues are not paired as $\pm \lambda_{i}$ ? In this case, let's assume the first $n$ eigenvalues are negative and all the rest $N-n$ eigenvalues are positive, then it's easy to see Eq. (2.68) is still valid, but the dimension of $\mathbf{C}_{1}$ becomes $n \times n$, the dimension of $\mathbf{C}_{2}$ becomes $n \times(N-n)$, dimension of $\mathbf{C}_{3}$ becomes $(N-n) \times n$, and dimension of $\mathbf{C}_{4}$ becomes $(N-n) \times(N-n)$. So our derivation for the nullifier vector still applies for this more general case.

Similarly, the derivation for the $\mathbf{Z}$ graph is also valid, we only need to change the dimension of $\hat{\mathbf{Q}}_{1}$ and $\hat{\mathbf{P}}_{1}$ to $n \times 1, \hat{\mathbf{Q}}_{2}$ and $\hat{\mathbf{P}}_{2}$ to $(N-n) \times 1$. Note the Fourier transformations can be applied on any $n$ modes, not necessarily the first $n$ modes.

What if all of G's eigenvalues are positive or negative (i.e., G is positive- or negative-definite)? In this case, Eq. 2.68) will have only $\hat{\mathbf{Q}}$ or $\hat{\mathbf{P}}$. Then no matter what local transformations are applied, the nullifiers can only be turned into the form $\hat{\mathbf{p}}-\mathbf{A} \hat{\mathbf{q}}$ where $\mathbf{A}=\mathbf{0}$, which means all the modes are not entangled with other modes, so this state is not useful.

The non-self-inverse $\mathbf{G}$ hasn't been studied for large scale states, because it's more complicated than the self-inverse case using the method in Ref. [17]. The method in 
this section can be used for finding non-trivial states generated from non-self-inverse $\mathcal{H}$ graphs.

\subsection{Summary}

In this Chapter, I have reviewed the basic ideas of qubits, qumodes and cluster states. I introduced three important graphs related to the cluster states: the A graph, the $\mathbf{Z}$ graph, and the $\mathcal{H}$ graph. I showed how to derive the $\mathbf{A}$ graph and the $\mathbf{Z}$ graph from a $\mathcal{H}$ graph, and extended the research to the non-self-inverse $\mathcal{H}$ graph, which haven't been studied yet.

In the next Chapter, I will introduce the fundamentals in the quantum optics implementation for generating cluster states. 


\section{Chapter 3}

\section{The Optical Parametric Oscillator}

In this chapter we introduce the heart of the optical continuous variable quantum computer: the optical parametric oscillator (OPO). The OPO is an optical cavity with nonlinear crystals inside. In order to understand the functionality of the OPO, we first talk about the optical cavities, and then the nonlinear optics, finally we combine these two to discuss about the $\mathrm{OPO}$ and the use in quantum computing.

\section{$3.1 \quad$ Optical Cavity}

Let first look at the simplest (but actually general) case, where the cavity consists of two mirrors, labeled 1 and 2 respectively. The input field goes to the cavity, part of it reflects, part of it transmits. The reflected field can be decomposed into a series of fields: the field reflected at the first incidence, the fields transmitted when the transmitted incident field bounces back for one round trip, two round trips, and so on. Similar treatment can be done for the transmitted field. 
The mathematical expression for the overall reflected field is:

$$
\begin{aligned}
E_{r} & =-r_{1} E_{0}+t_{1}^{2} r_{2} e^{i k d} E_{0}+t_{1}^{2} r_{2} e^{i k d} r_{1} r_{2} e^{i k d} E_{0}+\ldots \\
& =\frac{r_{2} e^{i k d}-r_{1}}{1-r_{1} r_{2} e^{i k d}} E_{0}
\end{aligned}
$$

where $E_{0}$ is the input beam, $k$ is the wave number, $d$ is the round trip length. Similarly, for the transmitted beam:

$$
\begin{aligned}
E_{t} & =t_{1} t_{2} r_{2} e^{i \frac{k d}{2}} E_{0}+t_{1} t_{2} r_{2} e^{i \frac{k d}{2}} r_{1} r_{2} e^{i k d} E_{0}+\ldots \\
& =\frac{t_{1} t_{2} e^{i k d}}{1-r_{1} r_{2} e^{i k d}} E_{0}
\end{aligned}
$$

The transmittance is then:

$$
\begin{aligned}
T & =\left|\frac{E_{t}}{E_{0}}\right|^{2} \\
& =\frac{t_{1}^{2} t_{2}^{2}}{1+r_{1}^{2} r_{2}^{2}-2 r_{1} r_{2} \cos (k d)}
\end{aligned}
$$

What if the cavity has loss? Assuming the loss is length dependent: $r_{l}=e^{\alpha d}$, then after one round trip, the beam will pick a factor of $r_{l} r_{1} r_{2} e^{i k d}$. We can actually absorb all reflective coefficients into a single parameter $r=r_{l} r_{1} r_{2}$. This trick works even the mirror has more than two mirrors, or more than one source of loss. Then the transmittance can be written as:

$$
T=\frac{1}{1+\frac{4 R}{(1-R)^{2}} \sin ^{2} \frac{k d}{2}}
$$

where $R=r^{2}$. This is a periodical function of $k d$. In experiments, usually we will fix either $d$ or $k$. Fixing $d$ means that the cavity mirrors are fixed and we're scanning 
the frequency of the input beam, fixing $d$ means that the input beam has constant frequency and the cavity length is scanned. Fig. 3.1 shows the transmittance versus the input frequency when $d$ is fixed. When $R$ increases, the peaks become narrower, indicating that approximately only a discrete set of frequencies can pass this cavity. The frequency difference between the top of peaks is called free spectral range (FSR) $\Delta$, the full-width half-maximum (FWHM) of each peak is called the linewidth $\delta$ of the cavity. From Eq. (3.4), we can easily get:

$$
\begin{aligned}
\Delta & =\frac{c}{d} \\
\delta & =\frac{c}{\pi d} \arcsin \sqrt{(1-R)^{2} / R}
\end{aligned}
$$

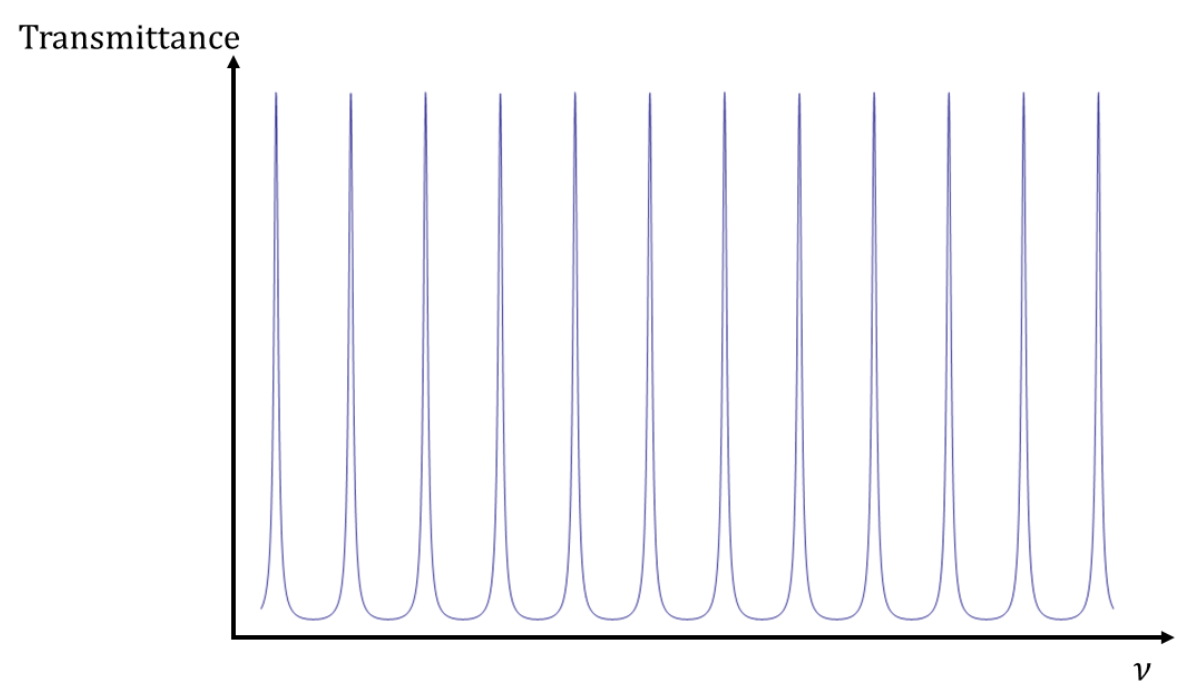

Figure 3.1: Cavity transmittance versus input frequency. 
The finesse is defined as: $\mathcal{F}:=\Delta / \delta$. In the case $T=1-R \ll 1$, we have:

$$
\begin{aligned}
\mathcal{F} & =\frac{\pi}{\arcsin \sqrt{(1-R)^{2} / R}} \\
& \approx \frac{\pi \sqrt{R}}{1-R} \\
& \approx \frac{2 \pi}{T}
\end{aligned}
$$

The final expression is very simple and useful for calculating finesse. For example, a one-sided cavity with a $(T=) 5 \%$ output mirror has finesse of $2 \pi / 0.05 \approx 126$, a two-sided cavity with two $5 \%$ mirrors has finesse of $2 \pi /(2 \times 0.05) \approx 63$.

\subsection{Nonlinear Optics}

\subsubsection{Introduction to nonlinear optics}

In linear optics, the relation between the electric field and the polarization density is $\mathbf{P}=\epsilon_{0} \chi \mathbf{E}$, where $\chi$ is a diagonal matrix for isotropic materials. For nonlinear materials, more terms are introduced

$$
\begin{aligned}
P & =P_{L}+P_{N L} \\
& =P_{L}+2 d E^{2}+4 \chi^{(3)} E^{3}+\ldots
\end{aligned}
$$

where $P_{L}=\epsilon_{0} \chi E$ is the linear term. The second and third term are called secondand third-order nonlinearity respectively. Usually, each successive order is weaker than the previous one, as long as the field isn't too large, so in here we only keep the

\footnotetext{
${ }^{1}$ This convention is used in Fundamentals of Photonics, B. E. A. Saleh and M. C. Teich, 1991. An alternative convention, $P=\epsilon_{0}\left(\chi E+\chi^{(2)} E^{2}+\chi^{(3)} E^{3}\right)$, is used in The Principles of Nonlinear Optics, Y. R. Shen, 1984.
} 
second order term.

By using Maxwell's equations, we can get the equation for fields in nonlinear materials:

$$
\nabla^{2} E-\frac{1}{c^{2}} \frac{\partial^{2} E}{\partial t^{2}}=\mu_{0} \frac{\partial^{2} P_{N L}}{\partial t^{2}}
$$

The only nonlinearity in this equation is the second order nonlinear term, which makes three-wave coupling possible. The three waves can propagate in different directions, in here, I first study the collinear case where three waves are all propagating along the $x$ axis.

To solve the above equation, we assume waves of three different frequencies are involved, then the solution can be written in the form [21]:

$$
\begin{aligned}
E(t) & =\sum_{j=1,2,3} \operatorname{Re}\left[E_{j} e^{i \omega_{j} t}\right] \\
& =\sum_{j=1,2,3} \frac{1}{2}\left[E_{j} e^{i \omega_{j} t}+E_{j}^{*} e^{-i \omega_{j} t}\right]
\end{aligned}
$$

where we assume $\omega_{3}=\omega_{1}+\omega_{2}$ and $\omega_{1} \neq \omega_{2}$. The nonlinear polarization density is:

$$
P_{N L}(t)=\frac{1}{2} d \sum_{j, k= \pm 1, \pm 2, \pm 3} E_{j} E_{k} e^{i\left(\omega_{j}+\omega_{k}\right) t}
$$

The right-hand side of Eq. (3.8) becomes:

$$
\mu_{0} \frac{\partial^{2} P_{N L}(t)}{\partial t^{2}}=-\frac{1}{2} \mu_{0} d \sum_{j, k= \pm 1, \pm 2, \pm 3}\left(\omega_{j}+\omega_{k}\right)^{2} E_{j} E_{k} e^{i\left(\omega_{j}+\omega_{k}\right) t}
$$

The final expression has 64 terms in total, but if we exclude the terms whose frequen-

\footnotetext{
${ }^{2}$ Note $c$ is the speed of light inside the material ( $c_{0}$ is the speed of light in vacuum), so the $P_{L}$ term is absorbed into the second term in the left-hand side of the equation.
} 
cies are not $\omega_{1}, \omega_{2}$ or $\omega_{3}$, then we have 36 terms left, with three frequencies. More specifically, we have:

(1) $\omega_{j}+\omega_{k}= \pm \omega_{3}$ in Eq. (3.11): $(j, k) \in\{(1,2),(2,1),(-1,-2),(-2,-1)\}$

(2) $\omega_{j}+\omega_{k}= \pm \omega_{2}$ in Eq. (3.11): $(j, k) \in\{(-1,3),(3,-1),(1,-3),(-3,1)\}$

(3) $\omega_{j}+\omega_{k}= \pm \omega_{1}$ in Eq. (3.11): $(j, k) \in\{(-2,3),(3,-2),(2,-3),(-3,2)\}$

So each case has four possible combinations.

Now we use the plane-wave approximation to write the fields as $E_{j}=A_{j} e^{-i\left(k_{j} x-\omega_{j} t\right)}$, $j \in\{1,2,3\}$. Under paraxial assumption, we have:

$$
\nabla^{2} E_{j}-\frac{1}{c^{2}} \frac{\partial^{2} E_{j}}{\partial t^{2}} \approx-2 i k_{j} \frac{d A_{j}}{d x} e^{-i k_{j} x}
$$

Plug everything into Eq. (3.8), we get:

$$
\begin{aligned}
& \frac{d a_{1}}{d x}=-i g a_{2}^{*} a_{3} e^{-i \Delta k x} \\
& \frac{d a_{2}}{d x}=-i g a_{1}^{*} a_{3} e^{-i \Delta k x} \\
& \frac{d a_{3}}{d x}=-i g a_{1} a_{2} e^{i \Delta k x}
\end{aligned}
$$

where $a_{j}=A_{j} / \sqrt{2 \eta_{j} \hbar \omega_{j}}, \eta_{j}=\eta_{0} / n_{j}$ is the impedance of the medium, $g^{2}=2 \hbar \omega_{1} \omega_{2} \omega_{3} \eta_{1} \eta_{2} \eta_{3} d^{2}$, and $d$ is the second-order nonlinear coefficient. The phase mismatching factor $\Delta k$ is:

$$
\Delta k=n\left(\omega_{3}, T\right) \frac{\omega_{3}}{c}-n\left(\omega_{1}, T\right) \frac{\omega_{1}}{c}-n\left(\omega_{2}, T\right) \frac{\omega_{2}}{c}
$$

From Eq. 3.13) we have:

$$
\frac{d}{d x}\left(\omega_{1}\left|a_{1}\right|^{2}+\omega_{2}\left|a_{2}\right|^{2}+\omega_{3}\left|a_{3}\right|^{2}\right)=0
$$


which indicates the conservation of energy. This is known as the Manley-Rowe relation 22 .

Depending on the pump frequency and beams' degeneracy, one can distinguish three common cases:

(1) $\omega_{1}=\omega_{2}$, so $a_{1}=a_{2}$, and the pump is $a_{1}$. Since the pump is much stronger than generated beams, so $\frac{d a_{1}}{d x}=0$ (undepleted pump approximation). Eq. (3.13) can be simplified, together with the energy conservation equation, the equations for all waves can be solved. This process is called second-harmonic generation (SHG). In the next subsection I will present the solution for SHG.

(2) Similar to the above case, let $a_{1}$ be the pump, however $\omega_{1} \neq \omega_{2}$. The equations can be solved in similar way, and this process is called sum frequency generation (SFG).

(3) The pump is $a_{3}$, so $\frac{d a_{3}}{d x}=0$. This is called parametric down-conversion (PDC).

In the next subsection, I will solve the equation for SHG. The other two cases, SFG and PDC, can be solved following the same procedure.

\subsubsection{Second-Harmonic Generation}

I first update Eq. (3.13) according to the conditions for SHG discussed in the previous subsection for the undepleted pumf $3^{3}\left(a_{1}=a_{2}, \frac{d a_{1}}{d x}=0\right)$ :

$$
\frac{d a_{3}}{d x}=-i \frac{g}{2} a_{1}^{2} e^{i \Delta k x}
$$

\footnotetext{
${ }^{3}$ The solution for the depleted pump case can be found at Chapter 19.4 in Fundamentals of Photonics, B. E. A. Saleh and M. C. Teich, 1991.
} 
where:

$$
\begin{aligned}
\Delta k & =n\left(\omega_{3}, T\right) \frac{\omega_{3}}{c}-2 n\left(\omega_{1}, T\right) \frac{\omega_{1}}{c} \\
& =\left[n\left(2 \omega_{1}, T\right)-n\left(\omega_{1}, T\right)\right] \frac{2 \omega}{c}
\end{aligned}
$$

Note in here the coefficient is half of that in Eq. (3.13), because according to Eq. (3.11), there are only two terms with frequency of $\omega_{3}:(j, k) \in\{(1,1),(-1,-1)\}$, instead of four.

Eq. 3.16 can be solved by integrating both sides, with the initial condition: $a_{3}(0)=0$ (i.e., no field of $\omega_{3}$ at the input side $\left.x=0\right)^{4}$ :

$$
a_{3}(L)=-i \frac{g}{2} a_{1}^{2} e^{i \frac{\Delta k L}{2}} L \operatorname{sinc} \frac{\Delta k L}{2}
$$

The intensity of field 3 is then given by:

$$
I_{3}(L)=2 \omega_{1}^{2} \eta^{3} d^{2} I_{1}^{2} L^{2} \operatorname{sinc}^{2} \frac{\Delta k L}{2}
$$

We have two observations from this solution:

(1) The intensity of field 3 is quadratic of the intensity of field 1.

(2) If $L$ (crystal length) is fixed, the maximum of $a_{3}(L)$ is achieved when $\Delta k=$ $k_{3}-2 k_{1}=0$, this is known as phasematching condition. For a fixed $L$, as $\Delta k$ increases, the intensity will drop rapidly since $\operatorname{sinc}(x)$ damps fast when $x$ increases.

We can also check the intensity of field 3 inside the crystal by varying $L$. Fig. 3.2 shows the intensity of the field 3 increases very fast (blue trace) as propagating along

\footnotetext{
${ }^{4}$ In here I used this notation: $\operatorname{sinc}(x)=\sin (x) / x$.
} 


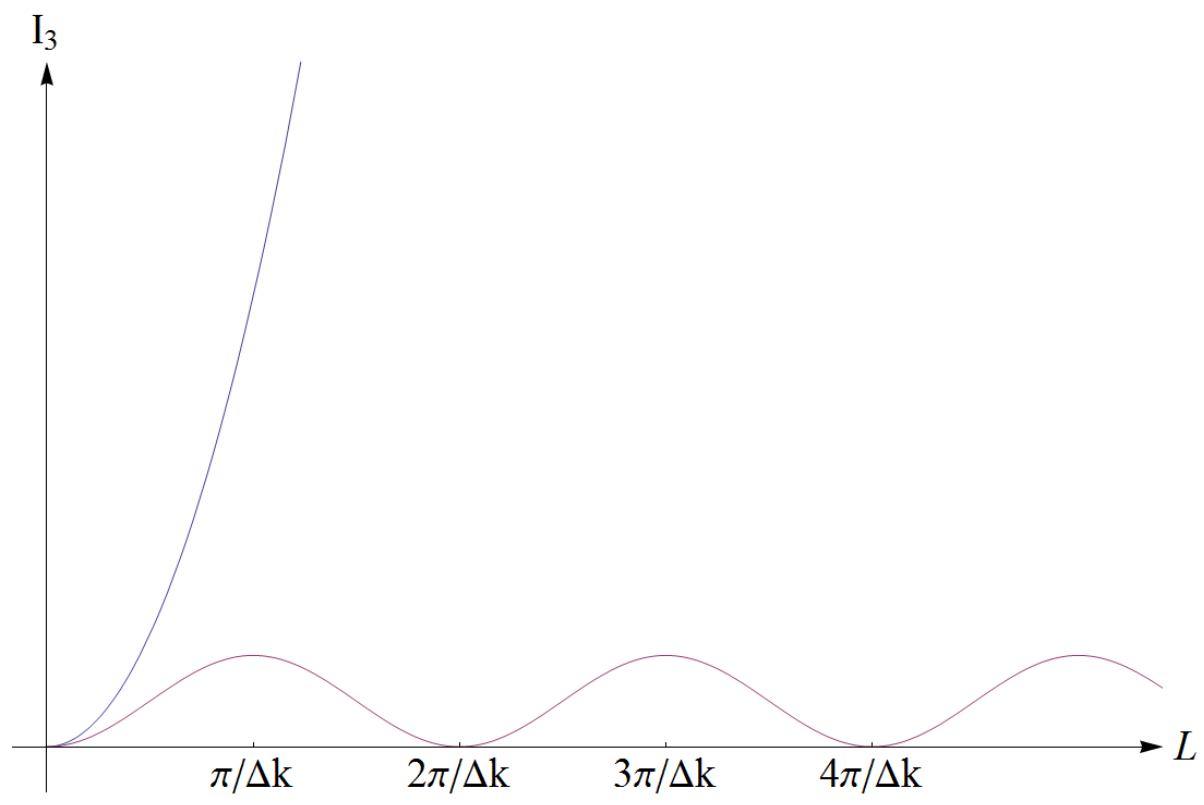

Figure 3.2: The blue trace is when the phasematching condition is satisfied, the red trace is when the condition is not satisfied.

the crystal when the phasematching condition is satisfied. When $\Delta k=0$, we have $I_{3}(L) \sim L^{2}$. If the phasematching condition is not satisfied, it will oscillate (red trace). In this case, we have $I_{3}(L) \sim \sin ^{2} \frac{\Delta k L}{2}$.

When this condition is not satisfied, the generated field $a_{3}(L)$ decreases rapidly. So to maximize the non-linear effect we usually need to fulfill the phase-matching condition.

\subsubsection{Phasematching}

Phasematching can be achieved by tuning the incident angle to the crystal and crystal temperature. Due to birefringence, the refractive index varies with propagating direction, for a certain choice of directions of the three fields, we can have $\Delta k=0$. This method of phasematching is called birefringent phasematching. Usu- 
ally the generated two fields will have different directions. Birefringent phasematching has better coupling strength, but the downside is the lack of tunability, the phasematching bandwidth is small, and it can only be applied for specific directions and frequencies.

Another phasematching method, known as quasi-phasematching, uses crystals of periodic structures, to compensate the momentum mismatching in the reciprocal lattice. A common method is known as periodic poling in ferroelectrics, where the second order nonlinearity is alternating between $d_{0}$ and $-d_{0}$ with a period of $\Lambda=$ $2 \pi / \Delta k$, where $\Delta k$ is the phase mismatching. Then second order nonlinearity can be written as a function of position and expended in Fourier series:

$$
d(x)=\sum_{m=-\infty}^{\infty} d_{0} \frac{2}{m \pi} e^{-i m \frac{2 \pi}{\Lambda} x}
$$

If we plug this to Eq. (3.13), the phase mismatching term will be compensated, but the effective nonlinearity is reduced by a factor of $2 / m \pi$, if the $m$ 'th order is used.

The nonlinear process will happen in a certain frequency range where the phasematching condition is satisfied. It is important to know how wide this range is, as we will discuss later, this range limits the number of entangled qumodes we can create. In the next section I present a study of the phase-matching bandwidth in a particular nonlinear crystal: periodically poled $\mathrm{KTiOPO}_{4}$ (PPKTP). 


\subsubsection{Quasi-phasematching bandwidth of PPKTP}

In general, the nonlinear coefficient $d$ is not isotropic, so I now write it in the tensor

form: $d_{i j}$, where $i \in\{1,2,3\}$, with $1,2,3$ referring to the $x, y, z$ axis of the crystal respectively, and $j \in\{1,2,3,4,5,6\}$, with $1,2,3,4,5,6$ referring to $x x, y y, z z, y z, z y, x z, x z, x y$ respectively [23]. For the interactions that are of most interest, $d_{33}$ corresponds to the $z z z$ process, means that pump field and two generated fields are all $z$ polarized. For KTP, the only three nonzero nonlinear coefficients are $d_{33}, d_{32}$ and $d_{24}$. In here, we study the phasematching bandwidth of $z z z$ PDC for PPKTP.

The phasematching bandwidth for PDC is the same as SFG, and experimental characterization for the latter is much easier, so I study the SFG instead of PDC, in both theory and experiment.

The system we want to model has two pumps at $\omega_{1}$ and $\omega_{2}$, due to SFG, field at $\omega_{3}=\omega_{1}+\omega_{2}$ will be generated, at the same time, SHG will also take place, so field at $2 \omega_{1}$ and $2 \omega_{2}$ will be generated as well. We still use the standard plane-wave approximation as we have used in the coupling wave equations. All classical fields 
are of the form $E_{j}=A_{j} e^{-i\left(k_{j} x-\omega_{j} t\right)}, j \in[1,5]$. The coupled wave equations are

$$
\begin{aligned}
& \frac{d a_{1}}{d x}=-i g_{1} a_{1}^{*} a_{4} e^{-i \Delta k_{1} x}-i g_{3} a_{2}^{*} a_{3} e^{-i \Delta k_{3} x} \\
& \frac{d a_{2}}{d x}=-i g_{2} a_{2}^{*} a_{5} e^{-i \Delta k_{2} x}-i g_{3} a_{1}^{*} a_{3} e^{-i \Delta k_{3} x} \\
& \frac{d a_{3}}{d x}=-i g_{3} a_{1} a_{2} e^{i \Delta k_{3} x} \\
& \frac{d a_{4}}{d x}=-i \frac{g_{1}}{2} a_{1}^{2} e^{i \Delta k_{3} x} \\
& \frac{d a_{5}}{d x}=-i \frac{g_{2}}{2} a_{2}^{2} e^{i \Delta k_{3} x}
\end{aligned}
$$

where $a_{j}=A_{j} / \sqrt{2 \eta_{j} \hbar \omega_{j}}, \eta_{j}=\eta_{0} / n_{j}$ is the impedance of the medium, $g_{1,2}^{2}=$ $4 \hbar \omega_{1,2}^{3} \eta_{1,2}^{2} \eta_{4,5} d_{33}^{2}, g_{3}^{2}=2 \hbar \omega_{1} \omega_{2} \omega_{3} \eta_{1} \eta_{2} \eta_{3} d_{33}^{2}$, and $d_{33}$ is the second-order nonlinear coefficient. The SHG and SFG phase mismatches are, respectively,

$$
\begin{aligned}
& \Delta k_{1,2}=n\left(2 \omega_{1,2}, T\right) \frac{2 \omega_{1,2}}{c}-2 n\left(\omega_{1,2}, T\right) \frac{\omega_{1,2}}{c}-\frac{2 \pi}{\Lambda}, \\
& \Delta k_{3}=n\left(\omega_{3}, T\right) \frac{\omega_{3}}{c}-n\left(\omega_{1}, T\right) \frac{\omega_{1}}{c}-n\left(\omega_{2}, T\right) \frac{\omega_{2}}{c}-\frac{2 \pi}{\Lambda},
\end{aligned}
$$

where $\Lambda$ is the poling period. In the limit of undepleted pumps $a_{1,2}, 15$ can be solved simply to yield the total intensity

$$
\begin{aligned}
I= & g_{3}^{2} L^{2}\left|a_{1} a_{2}\right|^{2} \operatorname{sinc}^{2}\left(\frac{\Delta k_{3} L}{2}\right)+\frac{g_{1}^{2}}{4} L^{2}\left|a_{1}\right|^{4} \operatorname{sinc}^{2}\left(\frac{\Delta k_{1} L}{2}\right) \\
& +\frac{g_{2}^{2}}{4} L^{2}\left|a_{2}\right|^{4} \operatorname{sinc}^{2}\left(\frac{\Delta k_{2} L}{2}\right) .
\end{aligned}
$$

We first examine the SFG phasemismatch of Eqs. (3.28 3.27) at room temperature as a function of the poling period $\Lambda$ and of SFG input — or OPO "signal" — frequencies $\omega_{1}$ and $\omega_{2}=\omega_{3}-\omega_{1}, \omega_{3}$ being fixed to the pump frequency (563 THz, $532 \mathrm{~nm}$ ) of the 
OPO. This can be done by using temperature-dependent Sellmeier equations 24,25]. The result, plotted in Fig. 3.3, displays a remarkably broad phasematching frequency bandwidth around frequency degeneracy $\left(\omega_{1}=\omega_{2}=\omega_{3} / 2\right)$. Therefore, we can expect a $532 \mathrm{~nm}$ pumped, $z z z$ PPKTP OPO to achieve mode entanglement over a $4.74 \mathrm{THz}$ full width at 99\% maximum, which would yield 10,000 entangled cavity modes for an OPO of $0.95 \mathrm{GHz}$ FSR.

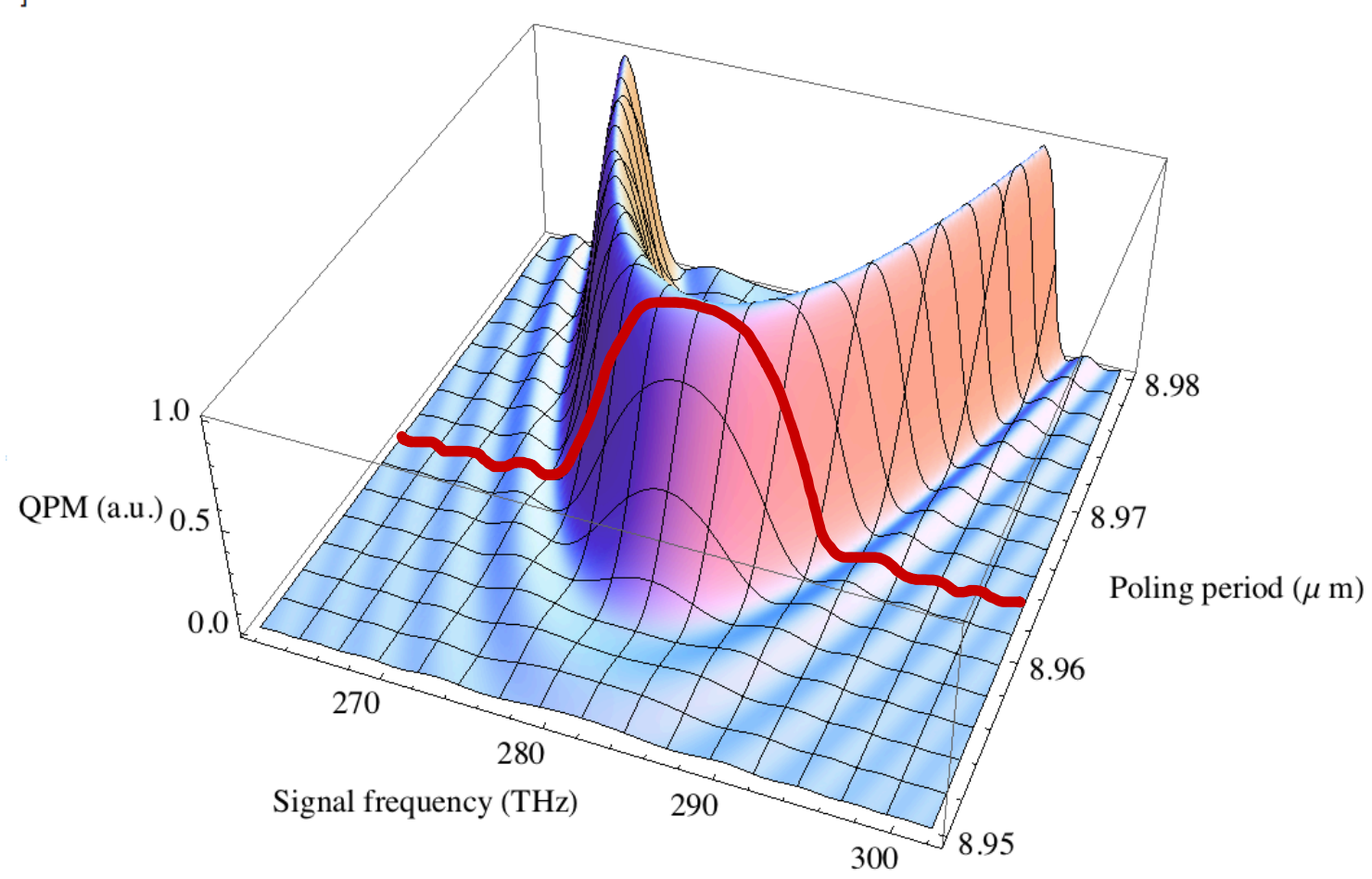

Figure 3.3: Quasiphasematching function, versus signal frequency $\omega_{1}$ and poling period, for a $1 \mathrm{~cm}$-long PPKTP crystal. The red line outlines the considered QPM FWHM bandwidth, which reaches $4.74 \mathrm{THz}$ at $99 \%$ of the maximum and decreases significantly in the separated branches.

Analogous calculations for longer wavelengths, such as $775 / 1550 \mathrm{~nm}$, indicate that the QPM bandwidth at $99 \%$ of the maximum should be broader by a factor of 3 .

In order to test this prediction, we used experimental SFG with 2 Newport-New 
Focus TLB-6721 "Velocity" tunable, continuous-wave, linearly polarized diode lasers, the sum of whose frequencies was kept constant over a 1058-1070 nm wavelength range. The experimental setup is shown in Fig. 3.4 The x-cut, $1 \times 2 \times 10 \mathrm{~mm}^{3}$

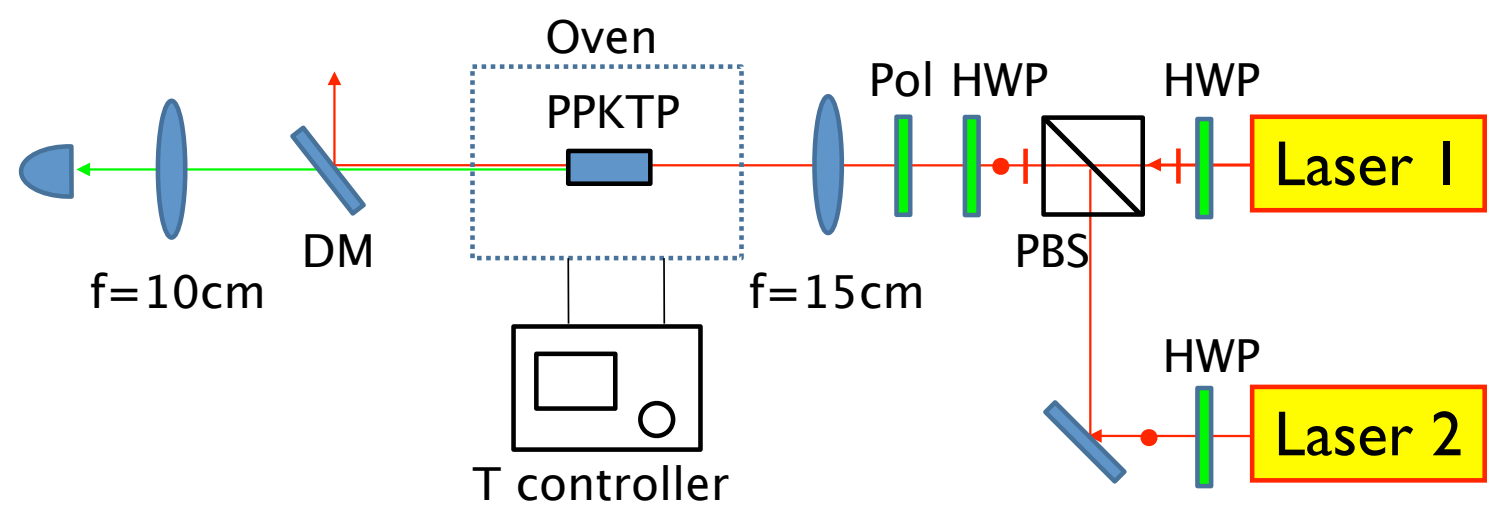

Figure 3.4: Experimental setup. HWP: half wave plate; PBS: polarizing beam splitter; Pol: polarizer; DM: dichroic mirror (HR for IR and AR for green).

PPKTP crystal was poled at a period of $9.0 \mu \mathrm{m}$ (at room temperature) and provided by Raicol Crystals. The crystal faces were antireflection coated by Advanced Thin Films for both $y$ and $z$ polarizations at $1064 \mathrm{~nm}$ and for the $z$ polarization at 532 $\mathrm{nm}$. The PPKTP crystal was placed in a temperature-stabilized oven, controlled by a Peltier module driven by a Wavelength Electronics, Model LFI-3751 temperature controller.

The power of the lasers are set to be 23.0(2) $\mathrm{mW}$ and 19.6(2) $\mathrm{mW}$, respectively, to compensate the efficiency difference caused by their different beam profiles. The power of the $z$-polarized beam entering the crystal are $7.7(2) \mathrm{mW}$ and $7.0(2) \mathrm{mW}$ respectively. Both lasers were scanned in the wavelength range of 1058-1070 nm, keeping the average wavelength of the two lasers fixed at 1064.090(5) nm, so that the 
generated SFG green beam always had $\lambda=532.05 \mathrm{~nm}$. The IR frequencies displayed by each laser were initially measured with a Coherent WaveMaster wavelength meter for calibration.

Figure 3.5(a) shows the measured power of generated green beam (both SFG and SHG) versus the fundamental wavelength and crystal temperature. For each temperature, about 30 data points of different wavelength are measured. The maximum SFG and SHG power were 19.2(1) $\mathrm{nW}$ and 8.94(1) $\mathrm{nW}$ respectively, yielding maximum efficiencies for SFG and SHG of $3.60(2) \times 10^{-4} \mathrm{~W}^{-1}$ and $1.67(1) \times 10^{-4} \mathrm{~W}^{-1}$, respectively. We attributed these relatively modest values to the unoptimized overlap of the highly elliptical transverse intensity profiles of the diode lasers. However, we believe it is reasonable to assume that this imperfect overlap was stationary throughout the measurements.

Figure 3.5(b) shows the theoretical prediction for the total green intensity. As can be clearly seen, the qualitative behavior of SHG (frequency-sharp ridges) and SFG (broadband feature) match the experimental results very well. The measured frequency width of the constant SFG QPM was 3.178(2) THz, at least, since our measurement was limited by the tuning range of the diode lasers (we could not make use of the full 1030-1070 nm tuning range of the lasers as it was not centered on the SHG fundamental wavelength of $1064 \mathrm{~nm}$ ). This measurement indicates that as many as 6,700 cavity modes (beyond the 60 actually measured) could be entangled in our recent demonstration in a single OPO [26]. 

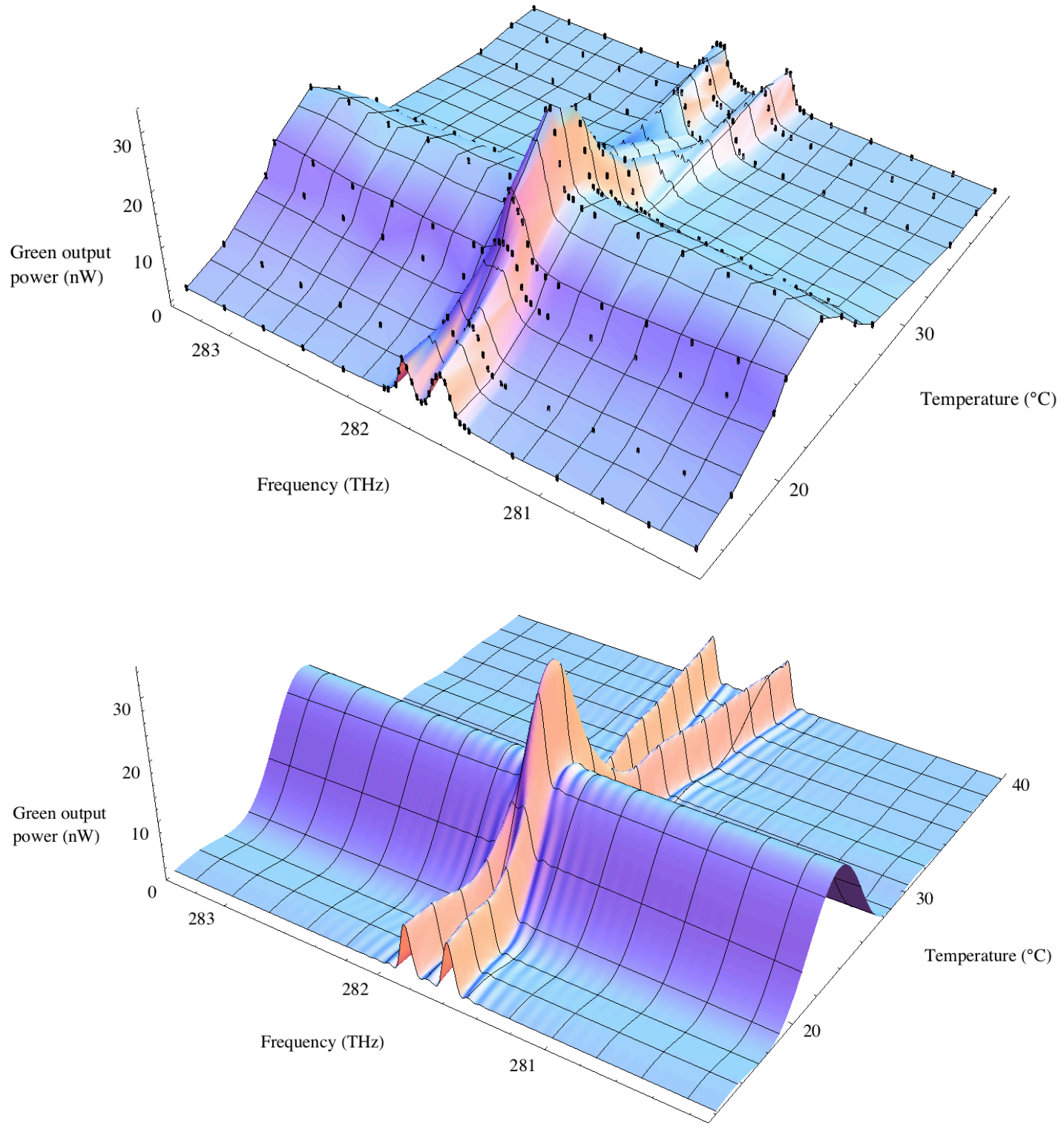

Figure 3.5: (a) Experimental phasematching curve. The laser wavelength is scanned from 1058 to $1070 \mathrm{~nm}$, the temperature of the crystal is scanned from $15^{\circ} \mathrm{C}$ to $40^{\circ} \mathrm{C}$ (11 different temperatures). About 30 data points of different wavelengths were measured at each temperature. The 3D plot was obtained by a linear interpolation (Mathematica) from the data points. (b) Theoretical phasematching curve, plotted by using the respective power values $18.28 \mathrm{nW}$ for $g_{3}^{2} L^{2}\left|a_{1} a_{2}\right|^{2}$, and $8.23 \mathrm{nW}$ for $g_{1}^{2} L^{2}\left|a_{1}\right|^{4}=g_{2}^{2} L^{2}\left|a_{2}\right|^{4}$ in Eq. 3.28). The measured SFG bandwidth is 3.178(2) THz, at quasi-constant efficiency, around $23^{\circ} \mathrm{C}$. The crossing ridges are due to the more narrowly quasiphasematched SHG interactions. 


\subsection{The Optical Parametric Oscillator}

Now what happens if we put the nonlinear crystal inside a resonant optical cavity? Let's focus on the PDC process, that is, we shine a pump beam into the cavity, and due to the nonlinear effect, the crystal will downconvert the pump field into pairs of fields that must satisfy the energy conservation and phasematching conditions, as in the previous Section. The difference is, instead of getting a continuous spectrum, only the modes at the resonant frequencies of the cavity will be enhanced by the cavity, all the other modes will be of much weaker intensity. As a result, we now have a discrete spectrum of modes, and they are created in pairs.

We already have a classical description of the nonlinear process, we now turn to the quantum one. The PDC process can be viewed in this way: one pump photon is annihilated, two signal photons are created, and their frequencies are restricted to the cavity resonant frequencies. So we can write the Hamiltonian as:

$$
\hat{H}=i \hbar \chi \sum_{i, j} \hat{b} \hat{a}_{i}^{\dagger} \hat{a}_{j}^{\dagger}+H . c .
$$

where $\hat{b}$ is the pump field and $\hat{a}_{i, j}$ are the generated fields. Since in PDC the pump field is very intense so we can treat it as a classical undepleted field: $\hat{b} \approx \beta$, take it out of the sum and define: $\kappa=\chi \beta$. Now this Hamiltonian becomes the Hamiltonian of two-mode squeezing, so this process will produce two-mode squeezed pairs, at the resonant frequencies. We represent this process in Fig. 3.6.

How many entangled modes do we have in such a device? As we have discussed 


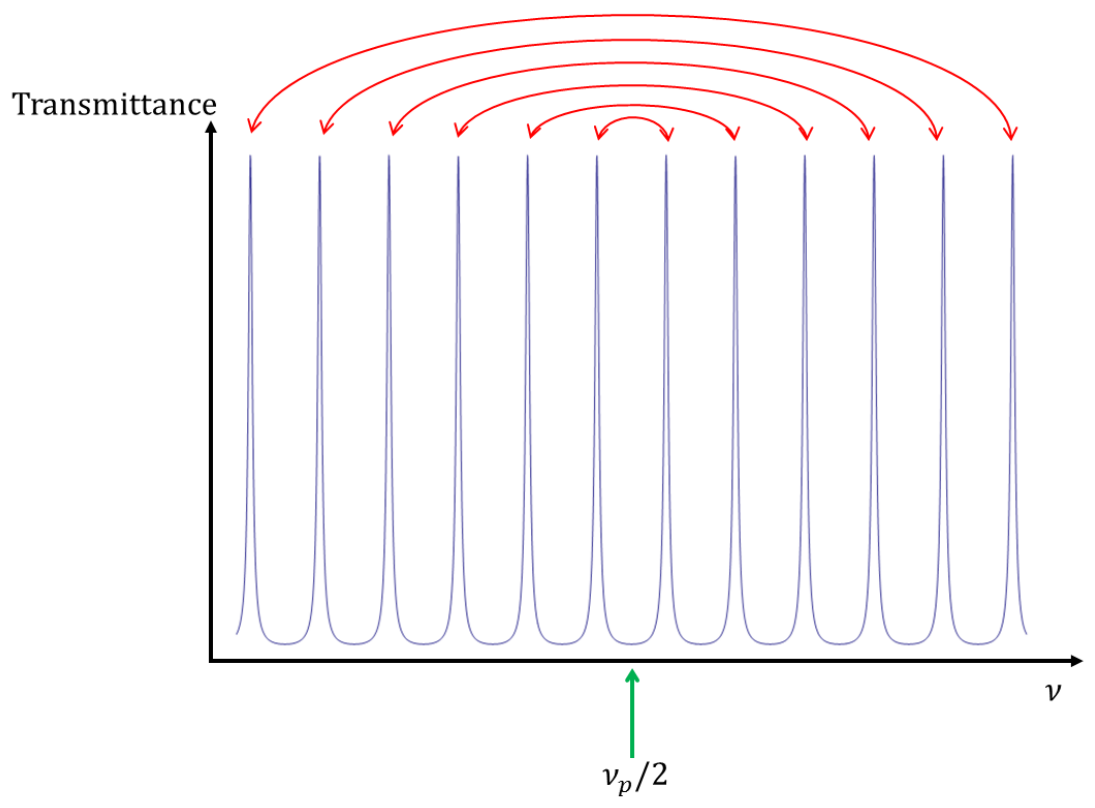

Figure 3.6: Pair generation by single pump.

in the previous section, the quasi-phasematching bandwidth of the nonlinear crystal limits the frequency range where PDC takes place. Now we have the cavity, only the resonant modes will survive. The pairs created by the PDC are always symmetric in frequency around half the pump frequency, however, it's not always the case that both modes in the pair will be enhanced by the cavity (doubly resonant). If one mode is not a resonant mode of the cavity (singly resonant or no resonant), the intensity of both modes will be much smaller. So the OPO dispersion also plays an important role, and we will talk about this in the next section.

\subsubsection{OPO dispersion}

Due to the dispersion of the nonlinear crystal of the OPO, we can expect the comb spacing to become uneven at frequencies far removed from half the pump frequency 
$\nu_{o}=\nu_{p} / 2$. Let $\Delta$ be the free spectral range (FSR) of the OPO; in the absence of dispersion, PDC will yield emission of photon pairs into, and generation of EPR entanglement between, qumodes at frequencies $\nu_{ \pm j}=\nu_{o} \pm j \Delta$, where $j$ is an integer. In the presence of dispersion in, say, a ring resonator, the FSR will depend on frequency, i.e. $j$ :

$$
\Delta_{j}=\frac{c}{L+n\left(\nu_{j}\right) \ell},
$$

where $L$ is the length of the air path in a round trip, $\ell$ is the length of the crystal path in a round trip, $n$ is the index of refraction of the crystal, and $c$ is the speed of light. In the presence of normal dispersion, we can expect the average frequency $\left(\nu_{j}+\nu_{-j}\right) / 2$ to veer away from $\nu_{o}$ as $j \gg 1$, which will lead to a severe degradation of squeezing as the shift $\left(\nu_{j}+\nu_{-j}\right) / 2-\nu_{o}$ becomes of the order of the OPO linewidth, see Fig. 3.7.

We now consider the exact OPO used in Ref. 26, contains two identical PPKTP crystals, oriented at $90^{\circ}$ from each other. With this arrangement, the OPO cavity is polarization degenerate and resonant frequency $\nu_{j}$ is given by (the refraction of air being negligible compared to that of KTP):

$$
\frac{2 \pi}{c} \nu_{j}\left[L+n_{z}\left(\nu_{j}\right) \ell+n_{y}\left(\nu_{j}\right) \ell\right]+\phi=j 2 \pi
$$

where $n_{z}\left(\nu_{j}\right), n_{y}\left(\nu_{j}\right)$ are the refractive indices for polarizations along the $z$ and $y$ principal axes of KTP crystal, respectively; and $\phi$ is the phase added by the OPO mirrors or other dispersion compensating elements. 


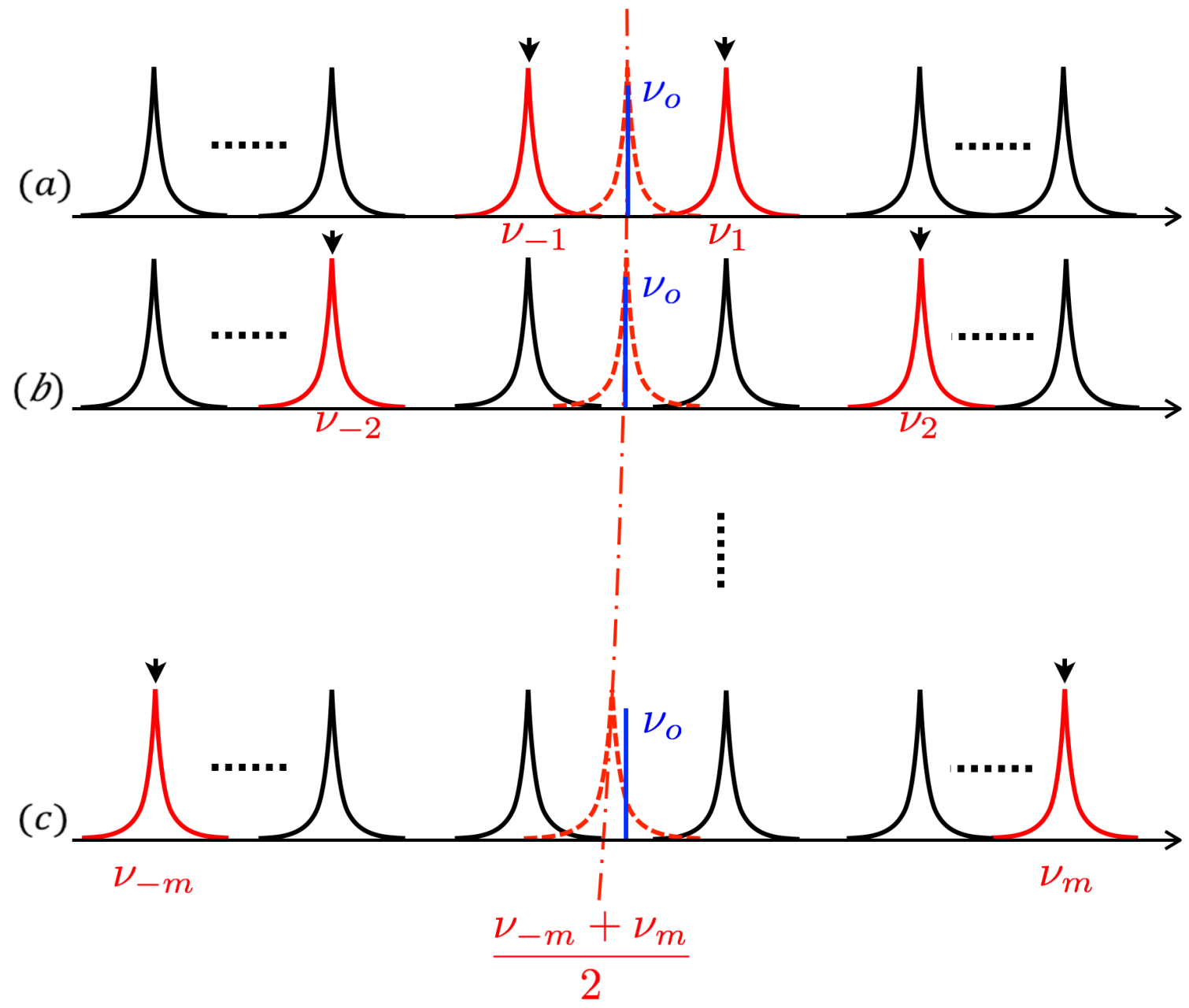

Figure 3.7: The dispersion effect of the OPO. The OPO pump has a fixed frequency and very narrow linewidth; the blue solid vertical line in the center marks half the pump frequency $\nu_{o}$. In the absence of dispersion, the symmetric resonant modes $\pm j$ of the OPO (solid lines) are equally spaced by a constant FSR: (a), $j=1$, $\nu_{o}=\left(\nu_{-1}+\nu_{1}\right) / 2 ;(\mathrm{b}), j=2, \nu_{o}=\left(\nu_{-2}+\nu_{2}\right) / 2$. In the presence of dispersion, i.e., for $j \gg 1$, the resonant modes of the OPO (solid lines) are not equally spaced by a constant FSR: $(\mathrm{c}), \nu_{o} \neq\left(\nu_{-j}+\nu_{j}\right) / 2$, the overlap between the PDC modes and the nearest OPO eigenmodes is severely reduced. 
If $\phi$ is frequency independent, which means no dispersion compensating is implemented in the OPO, then $j$ will have a constant offset of $-\phi$, which we set to zero for simplicity, so that:

$$
\nu_{j}=j \frac{c}{L+n_{z}\left(\nu_{j}\right) \ell+n_{y}\left(\nu_{j}\right) \ell} \equiv j \Delta_{j}
$$

where $\Delta_{j}$ is the dispersive FSR. Let $\nu_{j_{0}}$, which is half of the pump frequency, to be the center frequency (In our case, $\nu_{j_{0}}$ corresponds to $1064.04 \mathrm{~nm}$, our pump's wavelength is $532.02 \mathrm{~nm})$. Then Eq. (3.31) can be written as:

$$
\nu_{j}=\frac{\Delta_{j}}{\Delta_{j 0}} \nu_{j 0}+\left(j-j_{0}\right) \Delta_{j}
$$

Without dispersion, $\Delta_{j}$ is just the regular FSR, which is the same for all frequencies: $\Delta_{j}=\Delta_{j_{0}}$. The resonant frequencies $\nu_{j}^{\prime}$ satisfies: $\nu_{j}^{\prime}=j \Delta_{j_{0}}$, indicating that all the resonant frequencies are equally spaced by the FSR. With dispersion, the resonant modes are no longer equally spaced, because $\Delta_{j}$ is frequency dependent: $\Delta_{j} \neq \Delta_{j_{0}}$. Given an integer $j$, by solving Eq. (3.33), the correspondent resonant frequency $\nu_{j}$ can be obtained. The difference between $\nu_{j}$ and $\nu_{j}^{\prime}$ is the frequency shift due to dispersion $5^{5}$

To evaluate this effect, we used the Sellmeier equations of Ref. [27] for $n_{\text {air }}$, Ref. [24] for $n_{z}$, Ref. 28] for $n_{y}$, and we used the dependence determined in Ref. [25] for the temperature dependence of $n_{z}$ and $n_{y}$. For our OPO: $L=28.126 \mathrm{~cm}, l=1 \mathrm{~cm}$,

\footnotetext{
${ }^{5}$ In here, $\nu_{j}^{\prime}$ is defined as $\nu_{j}^{\prime}=j \Delta_{j_{0}}$. Notice that $\nu_{j}^{\prime}$ depends on choice of $\nu_{j 0}$. Within this paper, we fix $\nu_{j_{0}}$ to $1064.04 \mathrm{~nm}$.
} 
$\Delta_{j_{0}}=0.945 \mathrm{GHz}, \lambda_{j_{0}}=1064.04 \mathrm{~nm}$. The frequency shift referring to the regular frequency comb(where resonant frequencies are $\nu_{j}^{\prime}=j \Delta_{j_{0}}$ ) is shown in Fig. 3.8 .

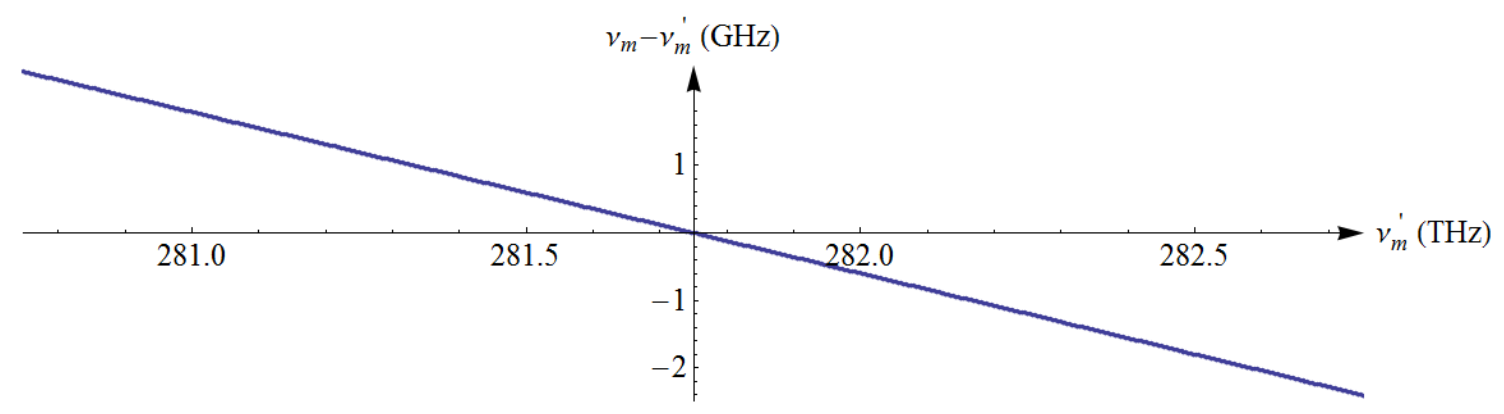

Figure 3.8: Frequency shift $\nu_{j}-\nu_{j}^{\prime}$ vs $\nu_{j}^{\prime}$, calculated by solving Eq. (3.33). Within the plot range $(200 \mathrm{GHz})$ it is almost linear and symmetric around the center frequency $\nu_{j_{0}}$.

The first order approximation of Eq. (3.33) provides a simple solution for the resonant frequencies with good precision. Since we only study the frequency range of $1 \mathrm{THz}$ around $\nu_{j_{0}}$, where the dispersion of air is $10^{-5}$ of the dispersion of the crystal, so we can treat it as a constant: $n_{\text {air }}\left(\nu_{j}\right)=1$. Then the Taylor expansion gives:

$$
n_{z, y}\left(\nu_{j}\right)=n_{z, y}\left(\nu_{j_{0}}\right)+\left.\left(\nu_{j}-\nu_{j_{0}}\right) \frac{d n_{z, y}}{d \nu}\right|_{j_{0}}+O\left[\left(\nu_{j}-\nu_{j_{0}}\right)^{2}\right]
$$

When:

$$
\left.\left.\left(\nu_{j}-\nu_{j_{0}}\right) \frac{d^{2} n_{z, y}}{d \nu^{2}}\right|_{\nu_{0}} \ll \frac{d n_{z, y}}{d \nu}\right|_{\nu_{0}}
$$

we can neglect the high order terms:

$$
\nu_{j}=j \frac{c}{L+n_{0} l+\left.l\left(\nu_{j}-\nu_{j_{0}}\right) \frac{d\left(n_{z}+n_{y}\right)}{d \nu}\right|_{\nu_{0}}}
$$

where $n_{0}=n_{z}\left(\nu_{j_{0}}\right)+n_{y}\left(\nu_{j_{0}}\right)$. Given $\left.l\left(\nu_{j}-\nu_{j_{0}}\right) \frac{d\left(n_{z}+n_{y}\right)}{d \nu}\right|_{\nu_{j_{0}}} \ll L+n_{0} l$, we can simplify 
it to:

$$
\begin{aligned}
\nu_{j} & =j \Delta_{j_{0}} \frac{1+\left.\frac{l}{c} \nu_{j_{0}} \Delta_{j_{0}} \frac{d\left(n_{z}+n_{y}\right)}{d \nu}\right|_{\nu_{j_{0}}}}{1+\left.\frac{l}{c} j \Delta_{j_{0}}^{2} \frac{d\left(n_{z}+n_{y}\right)}{d \nu}\right|_{\nu_{0}}} \\
& =j \Delta_{j_{0}} \frac{1-\frac{l}{j_{0}} D_{1}}{1-\frac{j l}{j_{0}^{2}} D_{1}}
\end{aligned}
$$

where $D_{1}=\left.\frac{d\left(n_{z}+n_{y}\right)}{d \lambda}\right|_{\lambda_{j_{0}}}$, and we used $\frac{d n}{d \nu}=\left(-\frac{c}{\nu^{2}}\right) \frac{d n}{d \lambda}, \nu_{j_{0}}=j_{0} \Delta_{j_{0}}$. Given $j l D_{1} / j_{0}^{2} \ll 1$, finally, we have:

$$
\begin{aligned}
\nu_{j} & =j \Delta_{j_{0}}\left[1+\frac{l}{j_{0}} D_{1}\left(\frac{j}{j_{0}}-1\right)\right] \\
& =\nu_{j_{0}}+m \Delta^{\prime}+m^{2} \frac{\Delta_{j_{0}} l D_{1}}{j_{0}^{2}}
\end{aligned}
$$

where $m=j-j_{0}, \Delta^{\prime}=\Delta_{j_{0}}\left(1+\frac{l}{j_{0}} D_{1}\right)$. This equation gives a very good approximate solution of Eq. (3.33).

When $m \ll j_{0}^{2} /\left(l D_{1}\right)$, the second order term is much smaller than the first order term, so:

$$
\nu_{j}=\nu_{j_{0}}+m \Delta^{\prime}
$$

where $\Delta^{\prime}=\Delta_{j_{0}}\left(1+\frac{l}{j_{0}} D_{1}\right)$. This equation shows the resonant modes are still equally spaced, but the spacing is $\Delta^{\prime}$, instead of $\Delta_{j_{0}}$ in the nondispersive case. Usually $D_{1}<0$, so $\Delta^{\prime}<\Delta_{j_{0}}$, which means all the resonant modes shrink to the center frequency mode $\nu_{j_{0}}$, comparing to the nondispersive case.

Notice that this conclusion does not depend on choice of center frequency $\nu_{j_{0}}$. Any resonant frequency can serve as $\nu_{j_{0}}$. A different $\nu_{j_{0}}$ will lead to a different nondispersive FSR $\Delta_{j_{0}}$, which gives a different regular frequency comb to define the 
frequency shift.

Also, from Eq. (3.39), we can see that from the $m$-th resonant mode referring to the center frequency, the integer $j$ increased by $m$. In other words, mode $\nu_{j_{0}+m}$ is the $+m^{\prime}$ th resonant mode from $\nu_{j_{0}}$. This is obviously true in nondispersive case, but does not hold when the second order term is comparable to the first order term, i.e., $m \sim j_{0}^{2} /\left(l D_{1}\right)$. We only study $1 \mathrm{THz}$ around the center frequency, where the dispersion effect is not very strong so this relation still holds. Then the $\pm m$ 'th modes from $\nu_{j_{0}}$ is just $\nu_{j_{0} \pm m}$.

We now check the average of $\nu_{j_{0}-m}$ and $\nu_{j_{0}+m}$, which are the $\pm m^{\prime}$ th modes from the center frequency $\nu_{j_{0}}$. From Eq. (3.38), we have:

$$
\left(\nu_{j_{0}-m}+\nu_{j_{0}+m}\right) / 2-\nu_{j_{0}}=m^{2} \frac{\Delta_{j_{0}} l D_{1}}{j_{0}^{2}}
$$

Typically, $j_{0} \sim 3 \times 10^{5}, D_{1} \sim-7 \times 10^{4} \mathrm{~m}^{-1}$, then the shift is around $-7.77 \mathrm{~m}^{2} \mathrm{~Hz}$. The deviation between this solution and the exact solution increases as $m$ increases, for $m \sim 320$, the deviation is only $500 \mathrm{kHz}$. The linewidth of the resonant modes is the linewidth of the OPO, which is also the linewidth of the average frequency.

We can see as the average decreases gradually, the overlap between the average frequency(whose linewidth is the the linewidth of the resonant modes, which is the OPO linewidth) and the pump becomes smaller. When this overlap is out of half the linewidth of either the pump or the average frequency, the PDC will be very weak, as shown in Fig. 3.7. It is equivalent to say, when the PDC generated pairs' frequency 
difference is large, they will not be enhanced by the OPO due to OPO's disperion. Let $\Delta \nu$ to be the greater value of the pump's and the OPO's linewidth, the number of total modes, with orthogonal polarization, are $1.44 \sqrt{\Delta \nu / 2}$. For the case in [26], the pump's linewidth is $1 \mathrm{kHz}$, the OPO's linewidth is $10 \mathrm{MHz}$, which corresponds to 3220 modes.

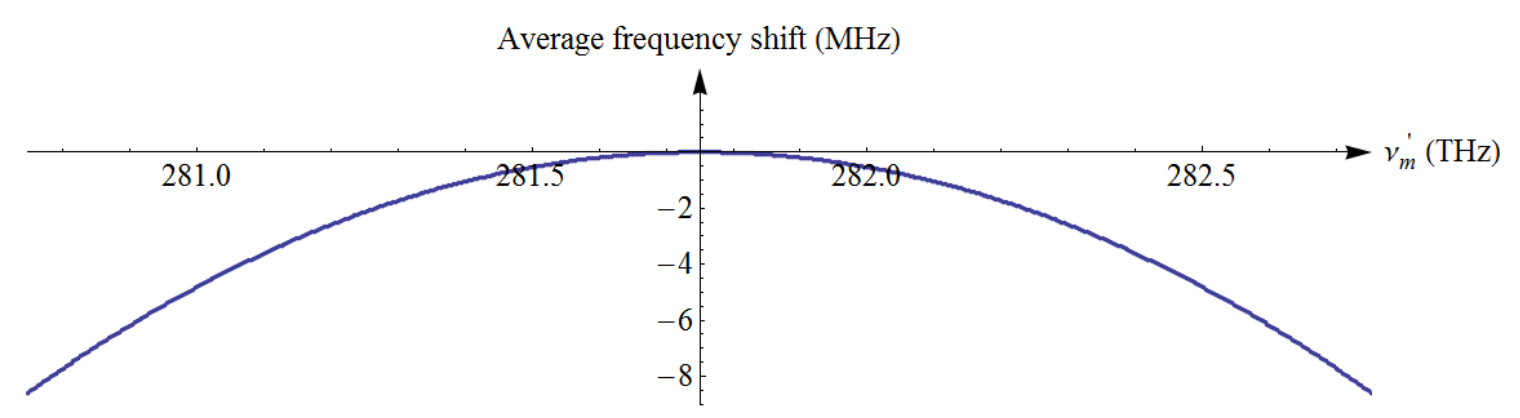

Figure 3.9: Average frequency shift vs $\nu_{j}^{\prime}$, calculated by solving Eq. (3.33). Equation (3.40) gives almost the same result over this frequency range.

Notice that in here we set $\phi=0$ in Eq. (3.31), however, by using dispersion compensating elements (for example, special coated mirrors, or prism pairs), this dispersion effect can be compensated.

\subsection{Conclusion}

In this Chapter, I have presented the basic components of the OPO and studied the limitation of the OPO as an entanglement source. For a $9 \mu \mathrm{m}$-poled PPKTP for $532 / 1064 \mathrm{~nm}$ up/down-converted wavelengths, the experiment shows a $3.178(2)$ THz QPM bandwidth, at constant SFG efficiency, and the result was limited by the tunability of our diode lasers and hence this result is only a lower bound for the actual 
QPM bandwidth. As it stands, this measurement indicates that as many as 10,000 cavity modes (beyond the 60 actually measured) could be entangled in our recent demonstration in a single OPO [26]. Analogous calculations for longer wavelengths, such as $775 / 1550 \mathrm{~nm}$, indicate that the QPM bandwidth at $99 \%$ of the maximum should be broader there by a factor of 3 .

Taking the OPO dispersion into consideration, I showed that the resonant modes become asymmetric around center frequency (half of the pump frequency) gradually, when the modes are off by the pump's linewidth, the generated pairs from PDC will not be enhanced by OPO. In the presence of dispersion, 3220 entangled modes can be generated using the scheme in [26]. Moreover, dispersion can be compensated by using dispersion compensating elements, such as prism compressors [29, 30], chirped mirrors [31,32], adding opposite group velocity dispersion materials [33, 34], and so on. These are widely used in mode-locked laser experiments. With the dispersion compensated, we expect 10,000 modes can be achieved in [26].

In this Chapter, I have only covered the case when a single pump is used in a OPO. What if we have more than one pump? We just need to add more terms into the OPO Hamiltonian. More generally, we can use a graph adjacency matrix $\mathbf{G}$ to represent the Hamiltonian's couplings, where the vertices are the generated modes, the edges indicate the entanglement, i.e., those modes are created as pairs. The Hamiltonian 
can then be written as:

$$
\hat{H}(\mathbf{G})=i \hbar \kappa \sum_{i, j} G_{i j} \hat{a}_{i}^{\dagger} \hat{a}_{j}^{\dagger}+H . c .
$$

which is Eq. (2.36). 


\section{Chapter 4}

\section{Generating Large Scale Cluster States}

I have presented the theoretical and experimental fundamentals in Chapter 2 and Chapter 3 respectively. In this Chapter, I will connect the results from the previous two chapters and introduce two important proposals that can generate large scale cluster states with rich structures for universal quantum computing.

\subsection{The Hypercubic Cluster States}

In this Section, we discuss the proposal for generating scalable hypercubic cluster states [4]. Up to 2012, most experimental proposals for making cluster states are "bottom-up" approaches, which is to entangle qubits one by one [6], which is usually hard to conduct in a scalable way. The only scalable "bottom-up" approach is to use temporal modes to sequentially generate cluster states [35, 36]. The only two "topdown" approaches are ultracold neutral atoms in optical lattice [37] and the quantum optical frequency comb (QOFC) from a single OPO [38, 39]. The two proposals, in Ref. [38] and Ref. [17], are the precursor of the work in this Section. At that time, 
creating large scale square-grid cluster states on QOFC in frequency domain was considered very hard, because the arrangement of qumodes in frequency domain is much more complicated than that in the time domain. In the time domain, at one time point only one entangled pair is created, and the "time" of one qumode can be easily changed by delaying 35. However, in the frequency domain, all entangled qumodes over the entire QOFC are created at the same time, and the frequencies of the qumodes can't be changed after creation. So to create a square-grid cluster state in the frequency domain, one has to make sure all the qumodes after creation are exactly at the desired frequencies.

In this section, I present a method for generating large scale hypercubic lattice cluster states in frequency domain. I first introduce the building blocks, the twomode squeezed states (TMSS), then talk about the lattice alignment, weaving and verification.

\subsubsection{Two-mode squeezed states generation}

The building blocks of the hypercubic state are the TMSSs, which are the entangled pairs generated by the OPO as introduced in Chapter 3. However, in Chapter 3 , we haven't utilized the polarization as a degree of freedom. In here, we use two identical PPKTP crystals oriented at $90^{\circ}$ from each other, with the first (second) quasiphasematching the $Z Z Z(Y Y Y)$ interaction, as defined by the polarization directions of pump and downconverted fields, $Z(Y)$ being the horizontal (vertical) direction. 
As we mentioned before, the QOFC created by the optical cavity is a collection of equally spaced, well-resolved qumodes. To make it easier to demonstrate, we label the modes by the frequency index $n \in \mathbb{Z}$ with in the comb, such that the resonant modes are at frequencies $\omega_{n}=\omega_{0}+n \Delta \omega$, with $\omega_{0}$ an arbitrary offset, and $\Delta \omega$ the FSR of the OPO cavity. Pump light at frequency $\omega_{\text {pump }}$ in the crystal will downconvert into photons of frequencies $\omega_{n_{1}}$ and $\omega_{n_{2}}$ such that:

$$
\omega_{\text {pump }}=\omega_{n_{1}}+\omega_{n_{2}}=2 \omega_{0}+\Delta \omega\left(n_{1}+n_{2}\right)
$$

We rewrite this phasematching condition by defining the pump index:

$$
p:=\frac{\omega_{\text {pump }}-2 \omega_{0}}{\Delta \omega}=n_{1}+n_{2}
$$

Nondegenerate downconversion, which creates TMS states with no single-mode squeezing, requires an odd pump index $p$ so that $n_{1} \neq n_{2}$. Without loss of generality, we assume that $n_{1}$ is odd and that $n_{2}$ is even from this point forward.

For convenience, we now replace each mode index with a macronode index $m:=$ $(-1)^{n} n$. The phasematching condition then becomes a difference condition on macronode indices: $p=m_{2}-m_{1}$. Since $m_{2}$ is assumed even and $m_{1}$ is assumed odd, we can repeatedly add 2 to both and still satisfy the condition. This relation therefore produces a two-step-translationally invariant set of interactions (for each polarization) with respect to the macronode indices [Figs. 4.1(a); 4.2(a); and 4.5(a), top].

We can write the Hamiltonian in the interaction picture with a single classical 


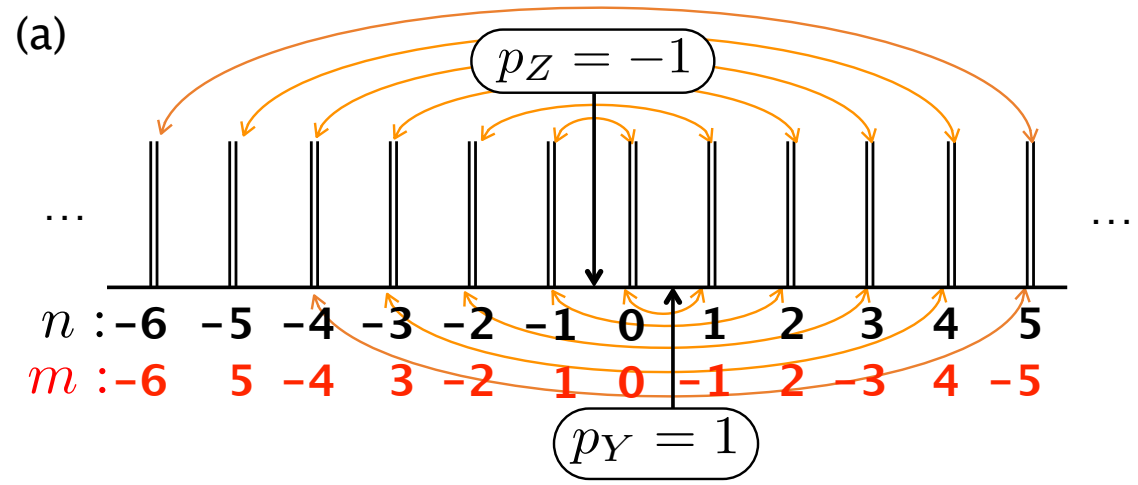

(b)

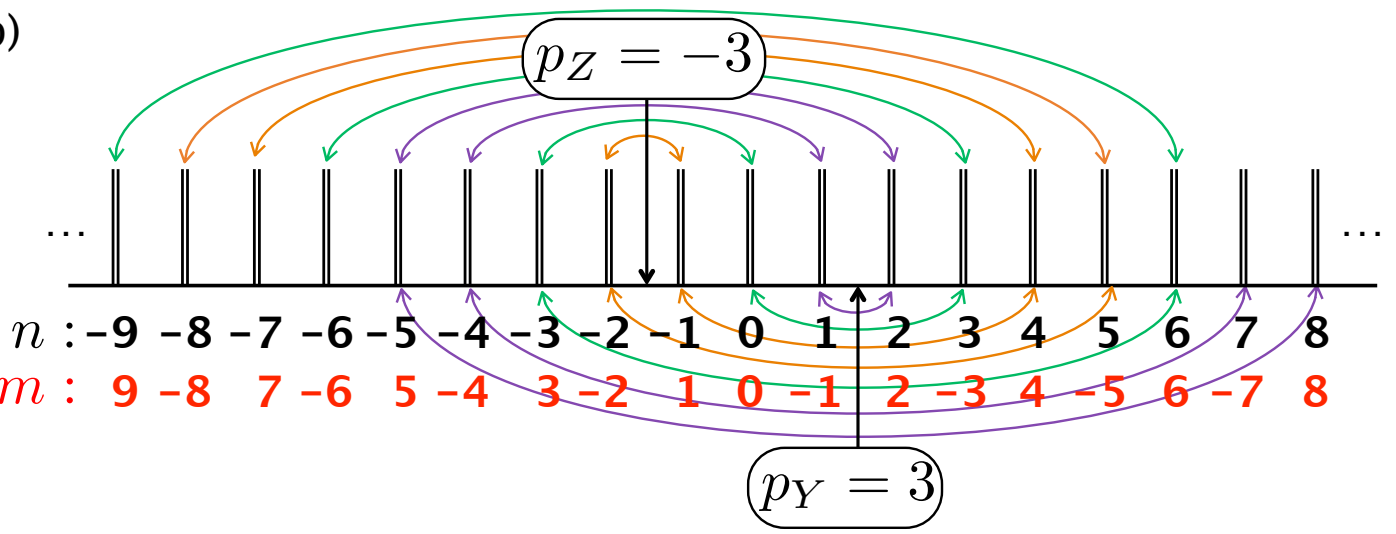

Figure 4.1: The phasematched QOFC interactions in two different OPOs, with $Y$ and $Z$-polarized pump indices $p_{Y}$ and $p_{Z}$ (black arrows). The qumodes are denoted by vertical lines (with orthogonal polarizations at the same frequency slightly separated for clarity) labeled by frequency index $n$ and node index $m$ (in red). The curved arrows denote the nonlinear interactions ( $Z Z Z$, top; $Y Y Y$, bottom), each of which becomes an edge of weight 1 in the OPO's $\mathcal{H}$ graph [17,39] and generates corresponding TMS states. (a) The $\mathrm{QOFC}$ of a single $\mathrm{OPO}$ with $p_{Y}=-p_{Z}=\Delta m=1$, which produces a single chain of interactions between adjacent node indices. (b) The QOFC of a single $\mathrm{OPO}$ with $p_{Y}=-p_{Z}=\Delta m=3$, which produces interactions between all pairs of node indices $m$ (red) separated by three units. This can also be interpreted as producing three independent chains (colored arrows) of the type obtained in (a).

undepleted pump. The well-known TMS Hamiltonian is:

$$
\hat{H}=i \hbar \kappa \hat{a}_{1}^{\dagger} \hat{a}_{2}^{\dagger}+\text { H.c. }
$$

where $\kappa>0$ is the overall nonlinear coupling strength. We can write this in terms of 
the adjacency matrix of a $\mathcal{H}$ graph $[17,39,41]$ :

$$
\mathbf{G}=\left(\begin{array}{ll}
0 & 1 \\
1 & 0
\end{array}\right)
$$

with components $G_{j k}$, as follows:

$$
\hat{H}[\mathbf{G}]=i \hbar \kappa \sum_{j k} G_{j k} \hat{a}_{j}^{\dagger} \hat{a}_{k}^{\dagger}+\text { H.c. }
$$

In this simple case, the two-mode interaction is purely nondegenerate (i.e., G is purely off-diagonal), and we have a graph with no self-loops. We will eschew degenerate interactions (self-loops in $\mathbf{G}$ ) throughout this paper. We now introduce more elaborate $\mathcal{H}$ graphs, which will be plugged into Eq. (4.5) to represent more complicated interactions.

\subsubsection{Macronode lattice setup}

We now show that the TMS states generated by $D$ OPOs are naturally arranged by the phasematching condition in a $D$-hypercubic lattice of frequency-degenerate macronodes. In the next Section, we will describe the interferometer that acts within each macronode to generate cluster entanglement.

\subsubsection{Scaling the graph valence}

We consider a collection of $D$ OPOs, each of which pumped by two monochromatic fields of distinct frequencies and orthogonal polarizations, with OPO \# $j$ having pump 


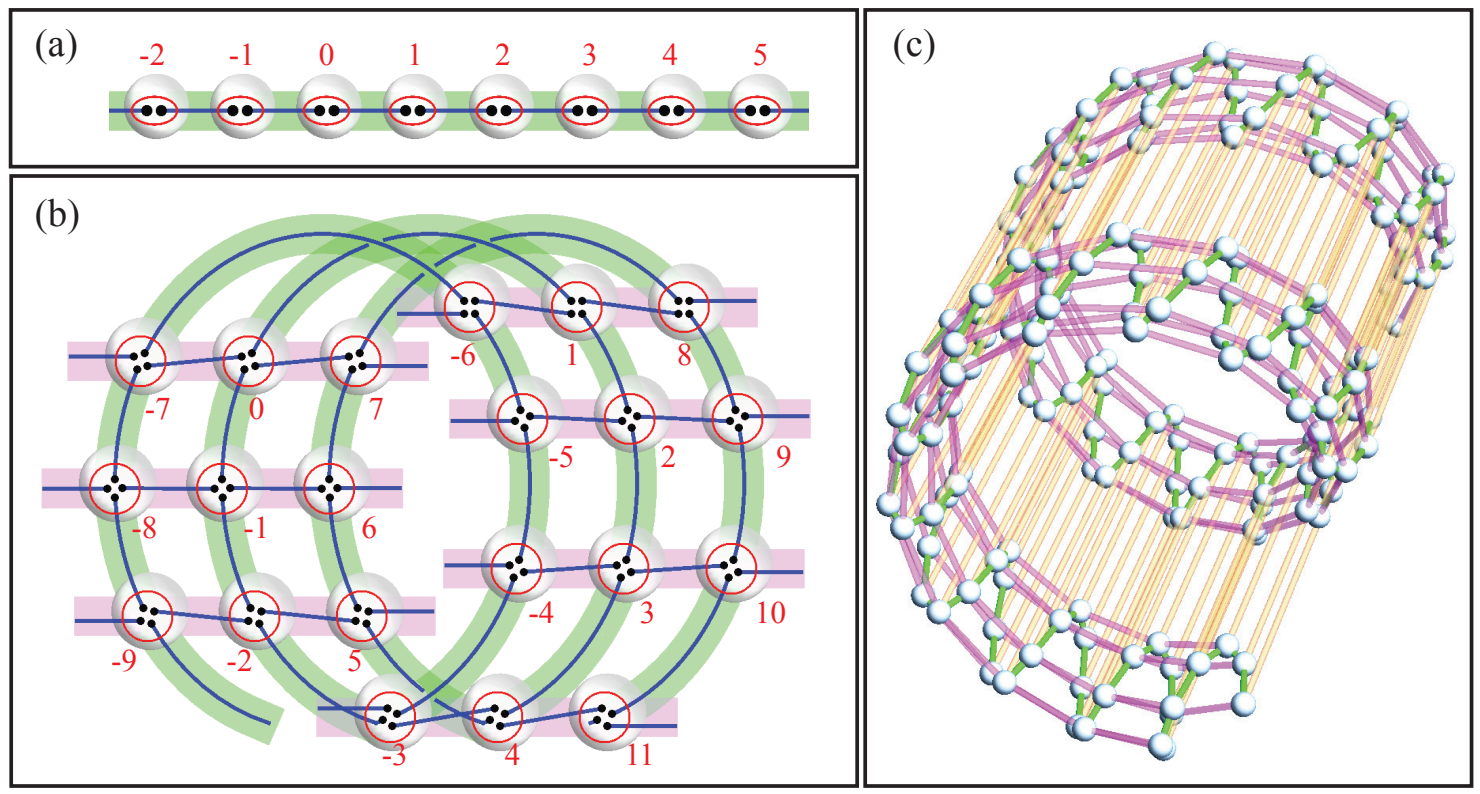

Figure 4.2: Arrangements of the TMS states qumodes into (a) linear, (b) squarelattice, and (c) cubic-lattice configurations (with $M_{2}=7$ and $M_{3}=13$ ) by grouping together frequency-degenerate qumodes into macronodes (red circles or white spheres) labeled by macronode indices (red numbers). See text for details. In (c), only the macronode connective structure is shown; individual qumodes and their connections are hidden for clarity. The macronode connections created by OPO \#1, \#2, \#3 are drawn in green, purple, yellow transparent tubes.

index $p_{j \varepsilon}$ per polarization $\varepsilon$. This implements the Hamiltonian

$$
\hat{H}=i \hbar \kappa \sum_{j=1}^{D} \sum_{\varepsilon \in\{Y, Z\}} \sum_{m_{j \varepsilon} \in 2 \mathbb{Z}+1} \hat{a}_{m_{j \varepsilon}}^{\dagger} \hat{a}_{m_{j \varepsilon}+p_{j \varepsilon}}^{\dagger}+\text { H.c. }
$$

which can be represented by $\hat{H}[\mathbf{G}]$ from Eq. 4.5) using the $\mathcal{H}$ graph:

$$
\mathbf{G}=\bigoplus_{j=1}^{D} \bigoplus_{\varepsilon \in\{Y, Z\}} \bigoplus_{m_{j \varepsilon} \in 2 \mathbb{Z}+1}\left(\begin{array}{ll}
0 & 1 \\
1 & 0
\end{array}\right)_{m_{j \varepsilon}, m_{j \varepsilon}+p_{j \varepsilon}}
$$

To create the desired structures, we prescribe that $p_{j Y}=-p_{j Z}=\Delta m_{j}$, which corresponds to an $\mathcal{H}$ graph with exactly one edge between all pairs of macronodes sep- 
arated by $\left|\Delta m_{j}\right|$, each of which produces a corresponding TMS state, as illustrated in Fig. 4.1 .

(1) Linear lattices: Figure 4.1(a) depicts the $\mathcal{H}$ graph of a single OPO (\#1) with $p_{1 Y}=-p_{1 Z}=\Delta m_{1}=1$ This graph is a collection of TMS state edges, which are shown reordered in Fig.4.2(a), where all qumodes of same index define to a macronode and a linear structure is clearly visible. (We will see later that a Hadamard interferometer transforms this linear sequence of disconnected EPR edges into a dual-rail quantum entangled wire, or single quantum wire over macronodes, as was experimentally demonstrated in Ref. [26].)

Figure 4.1(b) shows an additional, remarkable feature of this construction: when $\Delta m_{j}>1$, the OPO will generate $\Delta m_{j}$ (here, 3 ) disjoint quantum wires. This generation of multiple quantum wires in a single OPO was also demonstrated in Ref. 26 and is the basis for generating higher-dimensional lattices, to which we now turn.

(2) Square lattice: We now imagine taking the quantum-wire sequence of OPO \#1, as in Fig. 4.2(a), and "wrapping" it around a fictitious "cylinder," like a piece of thread around a spool [green wire in Fig. 4.2(b)]. We then employ a second OPO (\#2), with $p_{2 Y}=-p_{2 Z}=\Delta m_{2}=7$ here, to create 7 additional quantum-wire sequences [purple wires in Fig. 4.2(b)] whose macronodes exactly overlap with those of the first (spiraling) wire and bridge the spiral's coils with graph edges along the second lattice dimension (i.e., along the cylinder's axis), which will result in a square lattice with twisted cylindrical topology [Fig. 4.2(b)]. For a cylinder of circumference $M_{2}$ in units 
of macronode-index spacing, such a construction requires $\Delta m_{1}=1$ (for the wrapped wire) and $\Delta m_{2}=M_{2}$ (for the cross-links).

(3) Cubic lattice: This method can be extended to higher-dimensional lattices by using a fractal procedure, treating the twisted cylindrical lattice from the previous step as the linear resource to itself be wrapped around another cylinder [Fig. 4.2(c)], with an additional $\mathrm{OPO}$ used to create edges along the axis of the new cylinder and between adjacent macronodes along the new cylinder axis. For example, by first wrapping the wire around a cylinder of circumference $M_{2}$ and then wrapping that entire structure around a second cylinder of circumference $M_{3}$, we can create all the required macronode links with 3 OPOs with $\Delta m_{1}=1, \Delta m_{2}=M_{2}$, and $\Delta m_{3}=M_{2} M_{3}$. This results in a cubic lattice in the macronodes with twisted toroidal topology in the first two dimensions and linear topology in the third.

(4) Hypercubic lattices: Continuing this fractal progression weaves hypercubic lattices from macronodes. In general, for a $D$-dimensional hypercubic lattice, one employs $D$ OPOs with $\Delta m_{j}=\prod_{k=1}^{j} M_{k}$ for OPO \#j (and $M_{1}=1$ ). These lattices have twisted toroidal topology in the first $D-1$ dimensions and are linear in the $D^{\text {th }}$ one.

\subsubsection{Scaling the number of independent copies of the graph}

The same $D$ OPOs can create $M$ copies of a $D$-hypercubic lattice from (4) above, if OPO $\# j$ has pump indices: $p_{j(Z, Y)}= \pm M \Delta m_{j}+(M-1)$, and if we now label each macronode by a two-component compound macronode index $m^{(k)}$ for previous 
(a)

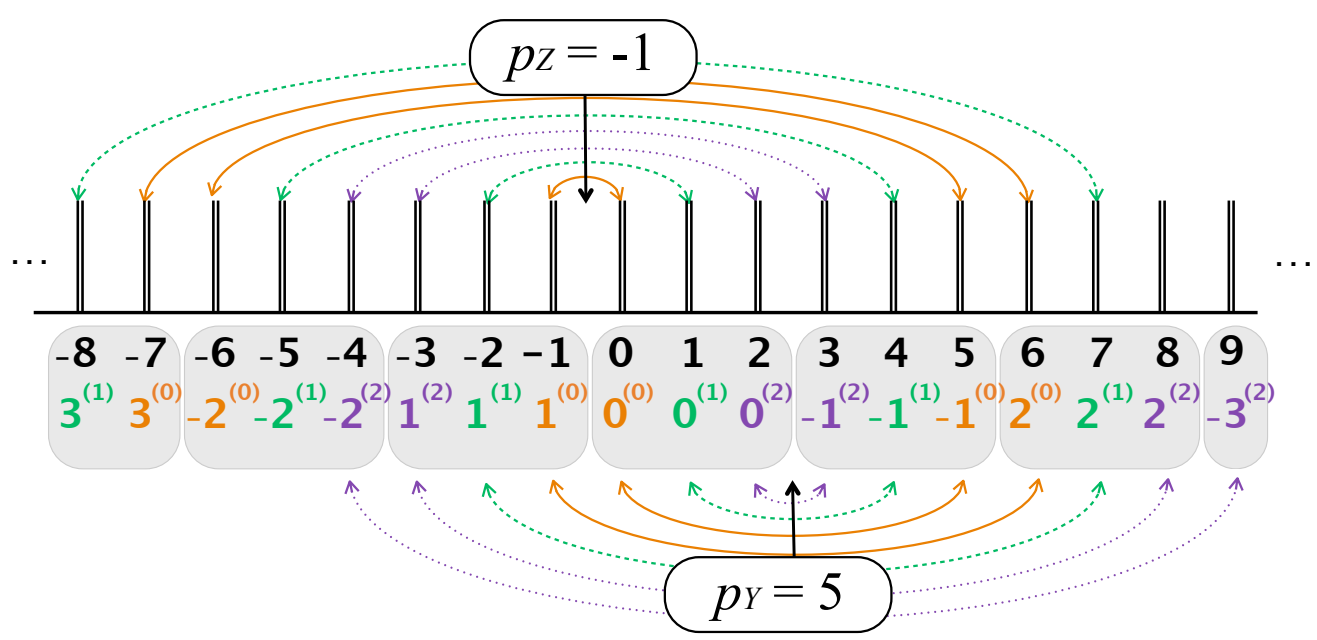

(b)

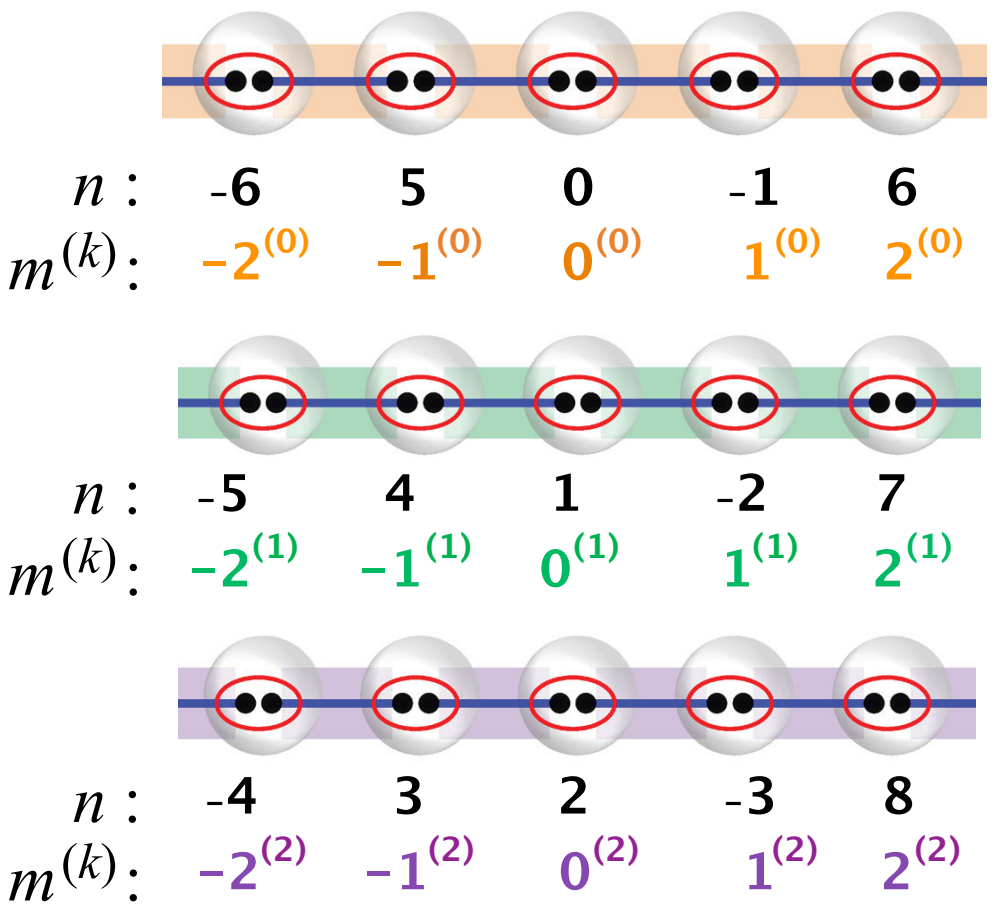

Figure 4.3: An example of making three copies of linear lattice cluster states. Different colors indicates different linear lattice cluster states. (a) The compound macronode index $m^{(k)}$ is used instead of the macronode index $m$. In this case, $\Delta m_{j}=1, M=3$, $k \in\{0,1,2\}$. (b) The TMS states can be arranged into three groups, each group will independently form a linear lattice cluster state. Starting from this, by applying the procedure from (2)-(4) multiple copies of lattice cluster states with higher dimension can be constructed. 
macronode index $m$ within lattice $k \in \mathbb{Z}_{M}$, then the frequency indices become:

$$
\begin{array}{ll}
n=\left(M m^{(k)}+k\right), & \text { if } m^{(k)} \text { is even } \\
n=-\left(M m^{(k)}+k\right)+(M-1), & \text { if } m^{(k)} \text { is odd. }
\end{array}
$$

An example of making three copies of linear lattice cluster states is shown in Fig. 4.3. Following the dimension building-up procedure from (2) to (4), multiple copies of square [Fig. 4.2(b)], cubic [Fig. 4.2(c)] and hypercubic lattice cluster states can be constructed.

\subsubsection{Macronode lattice entanglement}

With the quantum-wire sequences being appropriately arranged in a $D$-hypercubic pattern, we first describe the entanglement step, which is to interfere all qumodes within each (frequency-degenerate) macronode [35] by use of a Hadamard interferometer. The formal justification and proof of this will employ the graphical calculus for Gaussian pure states [17, 42].

\subsubsection{Experimental construction of hypercubic lattice clusters}

In the Heisenberg picture, the action of an interferometer on $2 D$ qumodes ( $D$ frequencies, two polarizations) is modeled by the action of a unitary matrix $\mathbf{U}$ on a vector of qumode annihilation operators $a=\left(\hat{a}_{1}, \ldots, \hat{a}_{2 D}\right)^{\mathrm{T}}$. Here, we need the interferometer to be balanced, i.e., all entries of $\mathbf{U}$ to have equal magnitude.

When $2 D$ is a multiple of 4 , up to 668 and possibly higher [43], $\mathbf{U}$ can be chosen to be a $2 D \times 2 D$ Hadamard matrix $\mathbf{R}$. We restrict ourselves to this case for simplicity, 
leaving the general case to future work. For $D=1$, a $\frac{\pi}{8}$ half-wave plate (HWP) acts as a balanced beamsplitter on polarization modes with $\mathbf{R}$, in this case, being:

$$
\mathbf{H}_{1}:=\frac{1}{\sqrt{2}}\left(\begin{array}{cc}
1 & 1 \\
1 & -1
\end{array}\right) .
$$

Using the Sylvester construction of Hadamard matrices [44, we can obtain the balanced $2 D$-splitter matrix: $\mathbf{H}_{D}:=\mathbf{H}_{1}^{\otimes D}$, which can be implemented using balanced beamsplitters [45, 46] or, equivalently, using $\frac{\pi}{8}$ HWPs and polarizing beamsplitters (PBSs). Figure 4.4 shows the experimental setup to generate cluster states with linear, square-lattice, and 4-hypercubic-lattice graphs. Each compact setup builds on the previous one, akin to the fractal construction of Fig. 4.2. All ring OPO cavities must be of identical FSR and held to the same exact resonant frequency, e.g., by Pound-Drever-Hall servo locks to the same counterpropagating reference laser beam 20,26$]$.

\subsubsection{Theoretical construction of hypercubic lattice clusters}

Any $N$-mode Gaussian pure state has a position-space wavefunction of the form 17

$$
\psi_{\mathbf{Z}}(\mathbf{q})=\operatorname{det}\left(\frac{\operatorname{Im} \mathbf{Z}}{\pi}\right)^{\frac{1}{4}} \exp \left(\frac{i}{2} \mathbf{q}^{\mathrm{T}} \mathbf{Z} \mathbf{q}\right)
$$

up to displacements, for some complex, symmetric matrix $\mathbf{Z}$ with $\operatorname{Im} \mathbf{Z}>0$. $\mathbf{Z}$ can be interpreted as the adjacency matrix of an $N$-node, undirected, complex-weighted graph and evolves under Gaussian unitary operations (in the Schrödinger picture) according to simple graph transformation rules [17]: Starting with the $D$ OPOs rep- 


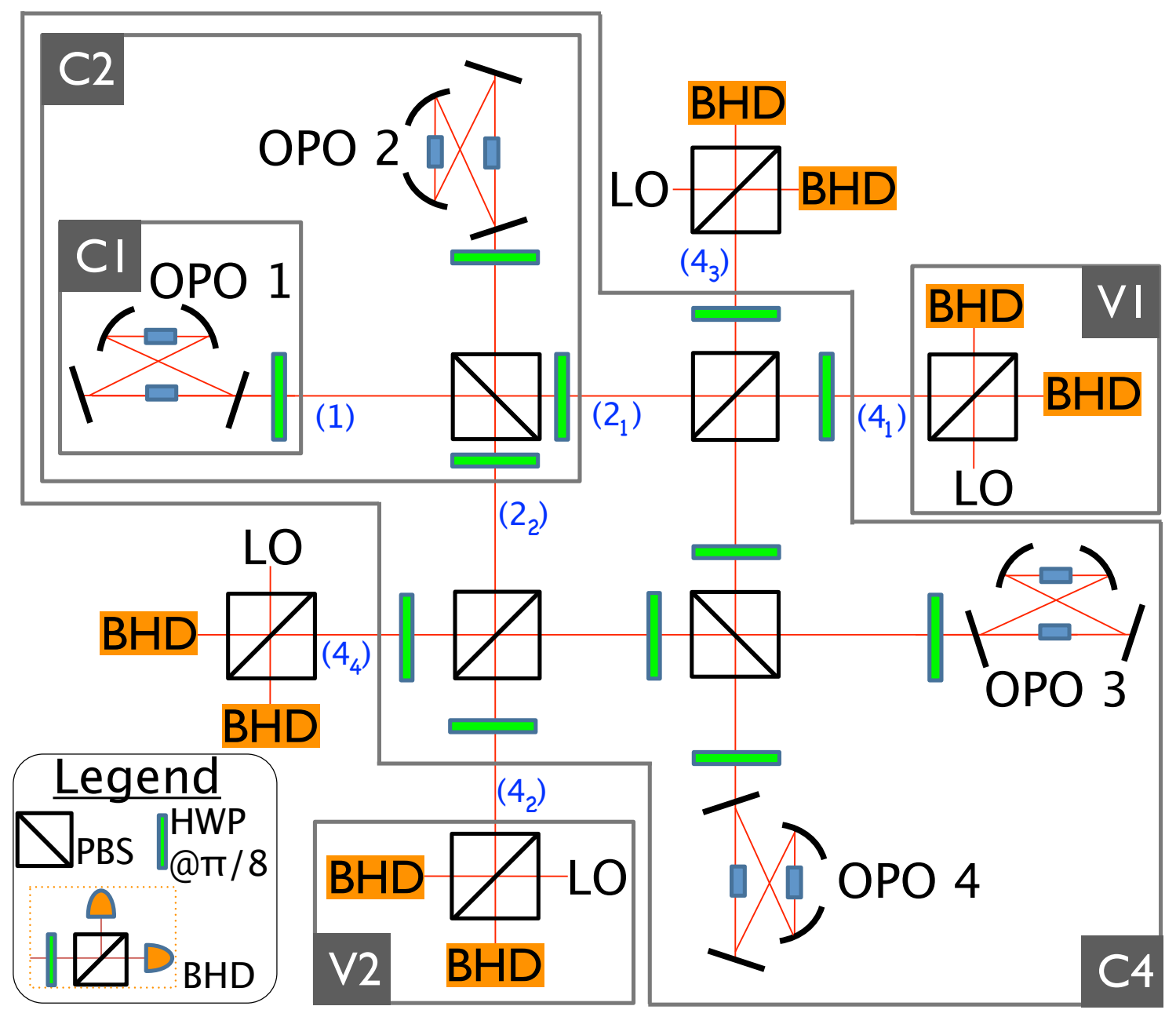

Figure 4.4: Compact experimental setups for generating and verifying QOFCbased CV cluster states with linear, square-lattice, and 4-hypercubic-lattice graphs. All polarizing beamsplitters (PBSs) transmit $Z$ and reflect $Y$, and all half-wave plates (HWPs) are at $\frac{\pi}{8}$ to the PBSs' axes. Box C1 generates at (1) a CV cluster state with linear topology as in Fig. 4.2(a) and graph structure as shown in Fig. 4.5.(a), which can be verified using two-tone balanced homodyne detection (BHD) in Box V1 (and omitting all the other optical elements). This was demonstrated experimentally in Ref. 26]. Box C2 builds on this setup to generate at $\left(2_{1,2}\right)$ a square-lattice CV cluster state with twisted cylindrical topology as in Fig. 4.2(b) and graph structure as shown in Fig. 4.5(b). This can be verified using Boxes V1 and V2. Box C4 further builds on this, generating at $\left(4_{1-4}\right)$ a 4-hypercubic-lattice CV cluster state with toroidal topology in the first three dimensions and linear topology in the fourth, which can be verified using all BHD's. The BHDs contain a two-tone local oscillator (LO), phaselocked to the OPO and polarized at $\frac{\pi}{4}$ to the PBS's axis 20,26]. 
resented by $\mathbf{G}$ from Eq. (4.7), when the Hamiltonian $\hat{H}(\mathbf{G})$ in Eq. (4.6) is applied on the vacuum state for time $t$, the output state is a Gaussian pure state with graph:

$$
\mathbf{Z}_{0}=i \exp (-2 \alpha \mathbf{G}),
$$

where $\alpha=2 \kappa t>0$ is an overall squeezing parameter. Crucially, since $\mathbf{G}$ is selfinverse [17,47], this relation simplifies to

$$
\mathbf{Z}_{0}=i c \mathbf{I}-i s \mathbf{G},
$$

where $c=\cosh 2 \alpha$, and $s=\sinh 2 \alpha$, resulting in a TMS state for each edge in G [Figs. 4.5(a), top, and 4.5(b), left].

We write the total interferometer as $\mathbf{R}=\bigoplus_{m \in \mathbb{Z}}\left(\mathbf{H}_{D}\right)_{m}$, which acts with $\mathbf{H}_{D}$ simultaneously on each macronode, evolving the state as 17

$$
\mathbf{Z}_{0} \stackrel{\mathbf{R}}{\longmapsto} \mathbf{Z}=i c \mathbf{I}-i s \mathbf{R G R}^{\mathrm{T}} .
$$

Since $\mathbf{R G R}^{\mathrm{T}}$ is self-inverse, $\mathbf{Z}$ is equivalent $[35]$ - up to trivial local phase shifts - to the approximate CV cluster state

$$
\mathbf{Z}_{C}=i \epsilon \mathbf{I}+t \mathbf{R G} \mathbf{R}^{\mathrm{T}},
$$

where $\epsilon=\operatorname{sech} 2 \alpha$, and $t=\tanh 2 \alpha$. We focus on $\mathbf{Z}$ rather than $\mathbf{Z}_{C}$ for experimental simplicity but still refer to the former as a "CV cluster state" because the phase shifts can be absorbed entirely into mode-wise quadrature redefinitions [35].

As shown in Fig. 4.5, we can see after interfering the $2 D$ output qumodes of the 
OPOs by the balanced $2 D$-splitter, all qumodes within each macronode are entangled with all qumodes in the neighbor macronodes, thus creating a $D$-dimensional lattice cluster state.

\subsubsection{State verification}

To verify the state, we need to measure the nullifiers. As we have already derived in the previous Section, the nullifier vector is:

$$
\hat{\mathbf{n}}_{\theta}:=\mathbf{R}^{\mathrm{T}} q_{\theta}-t \mathbf{G} \mathbf{R}^{\mathrm{T}} q_{-\theta}
$$

which is comprised of simultaneously commuting observables known as approximate nullifiers [17] or variance-based entanglement witnesses [19]. Since $\mathbf{R}$ acts locally on frequency-degenerate qumodes and since $\mathbf{G}$ links each node to exactly one other of a different frequency, each component of $\hat{\mathbf{n}}_{\theta}$ contains exactly two frequencies and can be measured by the two-tone balanced homodyne detection methods of Refs. 20,26]. The theoretical covariance matrix [17] of $\hat{\mathbf{n}}_{\theta}$ is given by

$$
\operatorname{cov}\left(\hat{\mathbf{n}}_{\theta}\right)=\frac{\epsilon}{2}(\mathbf{I}-t \mathbf{G} \cos 2 \theta),
$$

vanishing in the large-squeezing limit $\alpha \rightarrow \infty$. Each element of $\hat{\mathbf{n}}_{\theta}$ therefore has a theoretical variance of $\epsilon$ (i.e., sech $2 \alpha$ ) units of vacuum noise.

Further application of the massively entangled QOFC to quantum information processing will require separating the frequencies. We are investigating the use of quantum-optics grade arrayed waveguide gratings [48] and of virtually-imaged phase 
arrays [49], which have been successfully implemented in classical optical frequency combs [50].

\subsubsection{Conclusion for the hypercube proposal}

We have proposed novel hypercubic-lattice cluster states, highly scalable in size, graph valence, and number of copies of the state, and we have detailed their experimental generation and characterization with remarkably compact and proven technology 20,26]. The macronode-based implementation presented here and elsewhere [35] occurs naturally in quantum optics [36] and is becoming known to be a more efficient use of such cluster states for one-way quantum computing [5]. This work further motivates the development of a unified theoretical approach to macronodebased cluster states. Finally, the availability of large-scale, high-dimensional lattices invites theoretical and experimental investigations into the topological properties of these structures [51], including their high-dimensional incarnations [52]. 

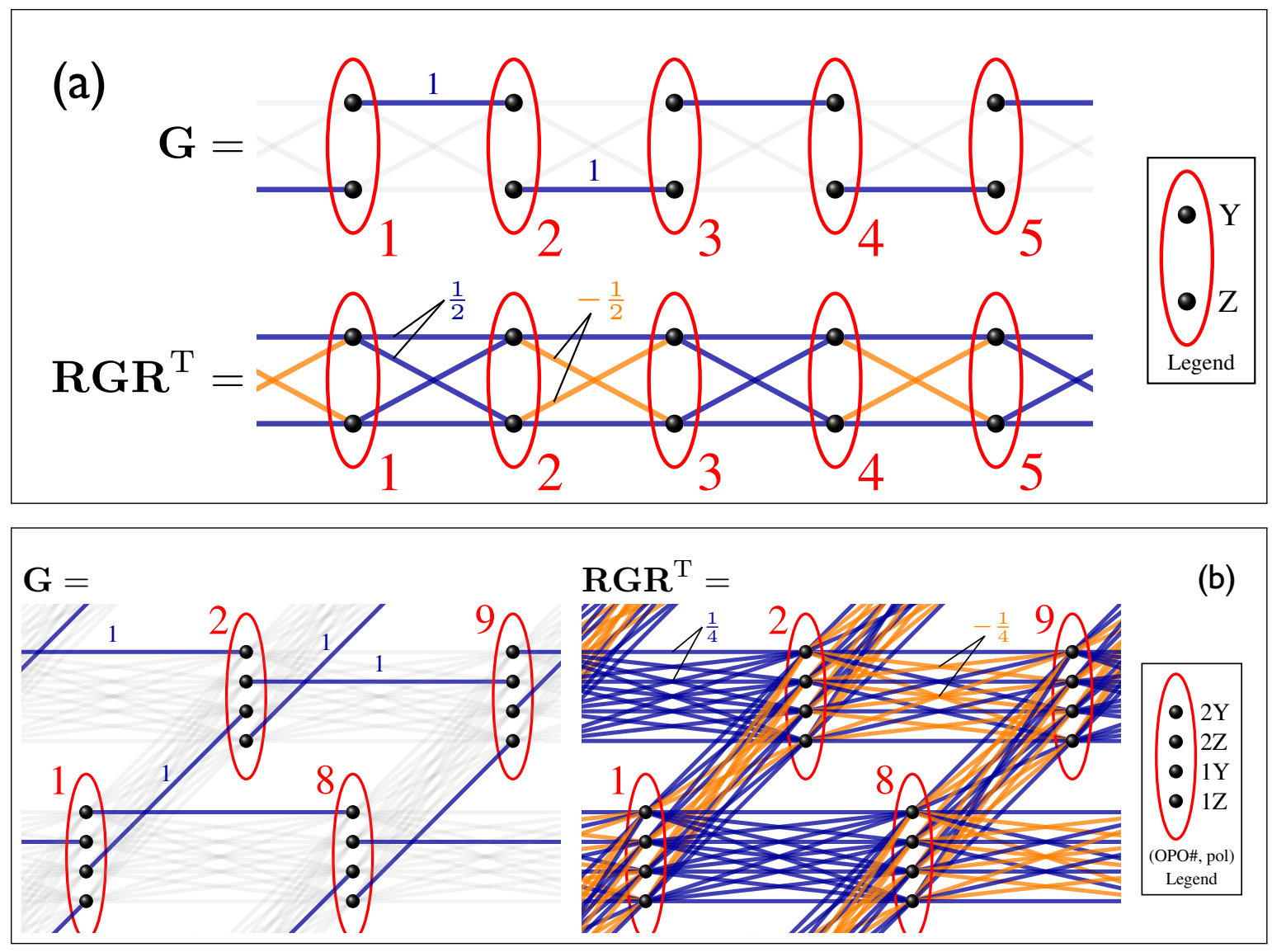

Figure 4.5: Effect of the interferometers acting on the macronodes. In both (a) and (b), the combined $\mathcal{H}$ graph $\mathbf{G}$ for the output of the $\mathrm{OPO}(\mathrm{s})$ is shown first, where the red circles indicate frequency-degenerate macronodes labeled by the red macronode indices (see text), with polarization [and $\mathrm{OPO} \#$ in (b)] as indicated in the legend. The state produced at the output of the OPOs has a graph [17] given by $\mathbf{Z}_{0}=i c \mathbf{I}-i s \mathbf{G}$ (see text), which corresponds to a collection of separable TMS states in accord with G. After the interferometer is applied (represented by the orthogonal matrix $\mathbf{R}$ ), a state with graph $\mathbf{Z}=i c \mathbf{I}-i s \mathbf{R} \mathbf{G R}^{\mathrm{T}}$ results, which is phaseshift equivalent to the CV cluster state $\mathbf{Z}_{C}=i \epsilon \mathbf{I}+t \mathbf{R} \mathbf{G} \mathbf{R}^{\mathrm{T}}$ (see text). The product $\mathbf{R G R}^{\mathrm{T}}$, interpreted as an adjacency matrix, is visualized as the second graph and provides an intuitive picture for the resulting state, as well as its precise definition through $\mathbf{Z}$ or $\mathbf{Z}_{C}$ [17]. (a) An $\mathcal{H}$ graph that is linear with respect to macronodes, a.k.a. dual-rail quantum wire [26], can be created from a single OPO with $\Delta m=1$ [Fig. 4.2(a)], and $\mathbf{R}$ represents the action of a balanced two-mode interferometer acting on each macronode. (b) An $\mathcal{H}$ graph with a square-lattice graph on macronodes can be created from two OPOs with $\Delta m=1$ and $\Delta m=M_{2}$ [Fig. 4.2(b)]. Here, $M_{2}=7$, and $\mathbf{R}$ represents the action of a balanced four-mode interferometer acting on each macronode. 


\subsection{The Time-Frequency Domain Cluster States}

In this Section, I introduce another proposal for generating ultra-scalable cluster states on the QOFC. Up to 2014, the one dimensional case of the two proposals in Ref. [35] (time domain) and Ref. [53] (frequency domain) have been demonstrated experimentally in Ref. [36 and Ref. 26] respectively, and both experiments set the world records in their own domains. However, both methods haven't reached their full potential. The time domain method only uses two frequency modes at one time, and the frequency domain method only uses the state at one time point. This suggests that if we use the scalability in both time and frequency, we can generate the most scalable cluster states that are at least four orders of magnitude larger than any other cluster states that have ever been created or proposed.

In this section, I present our proposal for generating time-frequency domain hybrid cluster states by utilizing the best of both proposals, with a single OPO [5]. Moreover, the state has simpler structure and are more entangled, comparing to previous square grid cluster state proposals.

\subsubsection{State generation}

The first step of this proposal is to generate the one dimensional dual-rail cluster state, as discussed in the last Section. The same OPO is used as shown in Fig. 4.6(a). A $\frac{\pi}{4}$ polarization rotation (by a halfwave plate at $\frac{\pi}{8}$ ) entangles the state into the frequency domain dual-rail quantum wires [4, 26], as shown in Fig. 4.6(b). Note that 
even if the pump beams are continuous wave $(\mathrm{CW})$, we can still logically assign pieces of the output beam to sequential time bins [36]. This leads to the idea that we can delay one "rail" of the dual-rail wire, and entangle it with the wire coming later. In this way, we will have a two dimensional state, the two dimensions are frequency and time. This idea will work, but will not create a clean square grid cluster state. Interested readers can verify this by applying the techniques I have introduced in previous sections.

We found that if only half of the frequencies in one "rail" of the dual-rail wire are delayed, the final state will be a clean square grid cluster state, as shown in Fig. 4.6(d). This requires us to separate the odd and even frequencies, and this can be done by using a Mach-Zehnder interferometer (MZI).

A MZI of path difference $c(2 \Delta \nu)^{-1}[54,55]$ separates frequencies of even and odd frequency index (and node index) into separate beams. For all odd qumodes, the $Z$ polarization is then time-delayed with with respect to the $Y$ polarization by the interval $\delta t$ between two consecutive time bins. The result is shown in Fig. 4.6(c). A final $\frac{\pi}{4}$ polarization rotation on the odd qumodes yields the BSL graph of Fig. 4.6(d).

A final phase shift by $-\frac{\pi}{2}$ (not shown) on either all odd or all even frequencies converts this into a finitely squeezed CV cluster state with the same ideal graph as in panel (d). It is this state that we call the BSL CV cluster state. The fact that the BSL is a bipartite, self-inverse graph makes this possible and ensures the scalability of the scheme $17,35,38,39,53$. (See the general discussion of bipartite, self-inverse 
graphs in Ref. 17.)

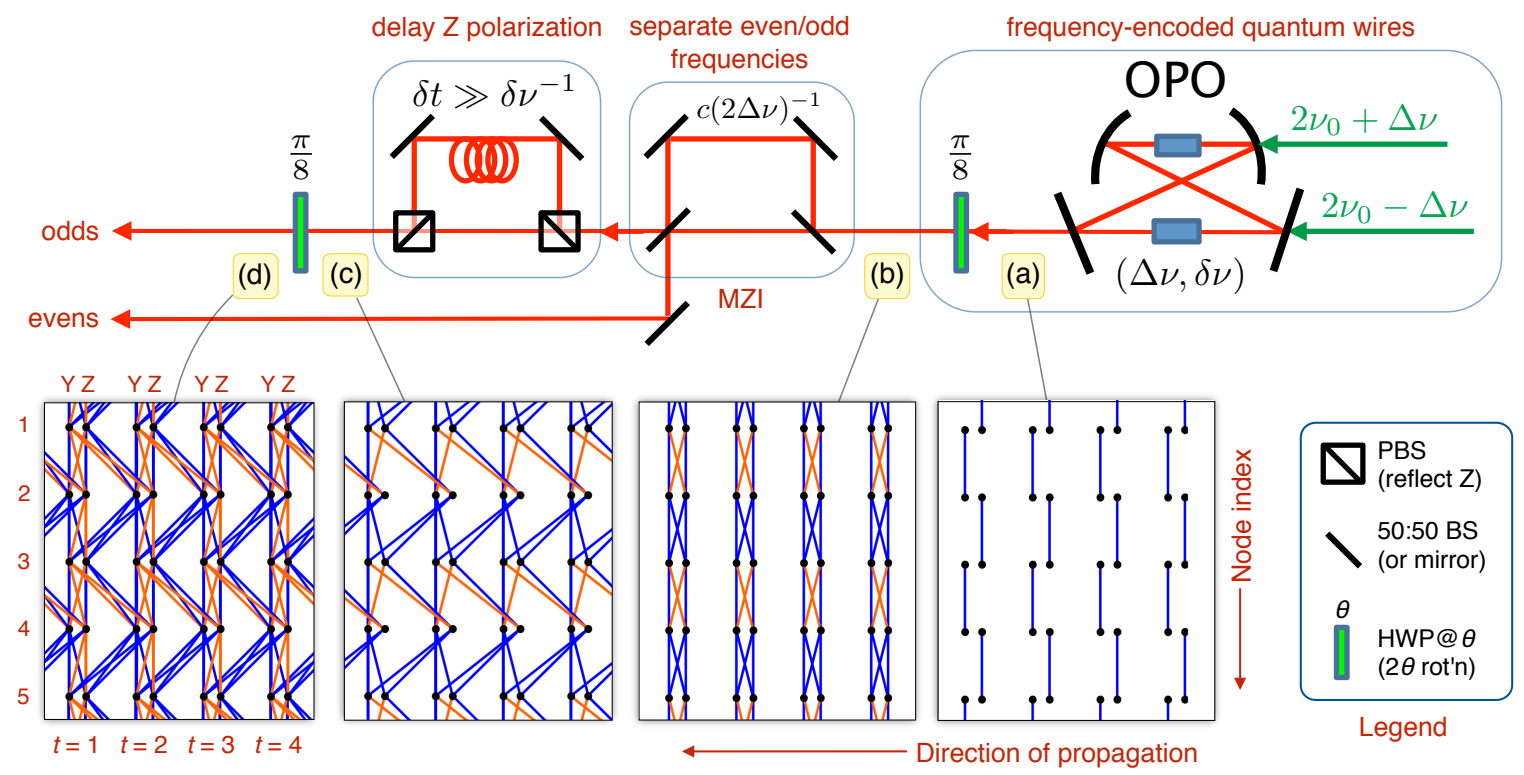

Figure 4.6: Experimental setup to generate a bilayer square-lattice (BSL) CV cluster state (see text for details). Abbreviations: $\mathrm{HWP} @ \theta=$ halfwave plate at angle $\theta$ to the horizontal axis (rotates polarization by $2 \theta) ;(\mathrm{P}) \mathrm{BS}=$ (polarizing) beamsplitter; MZI = Mach-Zehnder interferometer. Local oscillator fields, at the frequencies of the qumodes to be measured, will be injected at the unused input port of the MZI and will also be used for locking all optical phases in the experiment. Note that light propagates from right to left in the figure. The labeled panels show a precise graphical representation of the Gaussian state present in the beam at each step of the experiment, using the simplified graphical calculus for Gaussian pure states introduced in Ref. 35. Blue and orange correspond to edge weights of $\pm \mathcal{C}$ tanh $2 r$ [35], respectively, with $\mathcal{C}$ given below for each panel. All qumodes (black dots) are labeled as shown in the left panel: by node index (vertical) and by time bin and polarization (horizontal). (a) The OPO generates a temporal sequence of frequency-encoded twomode squeezed states $(\mathcal{C}=1)$. (b) Multiple (time-binned) dual-rail quantum wires encoded in frequency $[4,26]\left(\mathcal{C}=2^{-1 / 2}\right)$. (c) Result of delaying all odd-numbered $Z$-polarized qumodes $\left(\mathcal{C}=2^{-1 / 2}\right)$. (d) Final BSL CV cluster state $\left(\mathcal{C}=2^{-3 / 2}\right)$ after required phase shifts (see text).

There are some experimental constraints in the proposal. First, we have a "musical score" condition - i.e., the measurement times must be compatible with resolving all qumode frequencies: $\delta t \gg \Delta \nu^{-1}$, an easily fulfilled condition. In addition, the 
measurement times must allow one to achieve maximum squeezing - that is, they must be at least as long as the OPO cavity storage time [56]. This translates into $\delta t \gg \delta \nu^{-1} \gg \Delta \nu^{-1}$, since $\delta \nu$ is also half the squeezing bandwidth [57]. This condition can also be fulfilled in practice [36] and ensures that the time bins contain maximally squeezed qumodes, to the extent permitted by the experiment's squeezing limit (mainly determined by the intracavity losses).

The verification, i.e., measurements of nullifiers, can be done in the similar way as shown in Ref. 26, 53].

\subsubsection{Quantum computing}

We have looked at how to make a time-frequency domain square-grid cluster state, now we look at how to do quantum computing with this state. The BSL CV cluster state is easily shown to be universal for MBQC. Simply measure $\hat{q}$ on all qumodes of one (e.g., $Y$ ) polarization (which will delete the entire layer, as we discussed in Chapter 2), resulting in a CV cluster state with an ordinary square-lattice graph, which can be used with standard CV MBQC protocols [14, 58]. This is shown in Fig. 4.7(a). We can see in this method, half of the graph nodes are wasted. This method also inefficiently uses available squeezing, which leads to extra noise when using these resources for quantum computing [5]. Furthermore, lattice edges are at $45^{\circ}$ to the direction of increasing temporal index, meaning that either the information must flow in a zig-zag pattern or the lattice width will have to scale linearly with the length of the computation, hindering the scheme's scalability. 


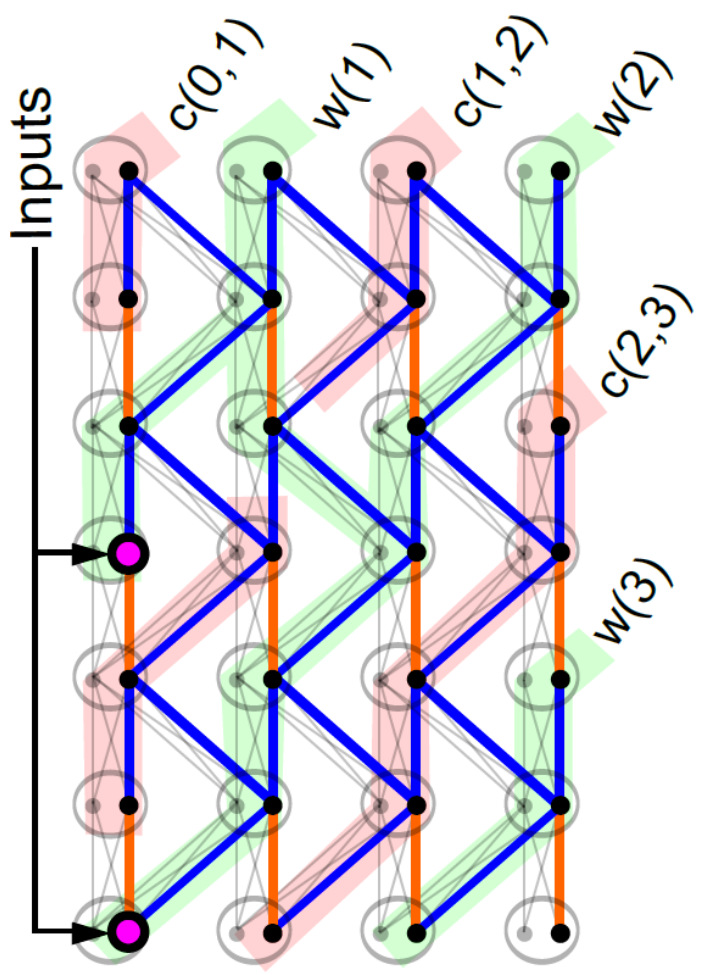

(a)

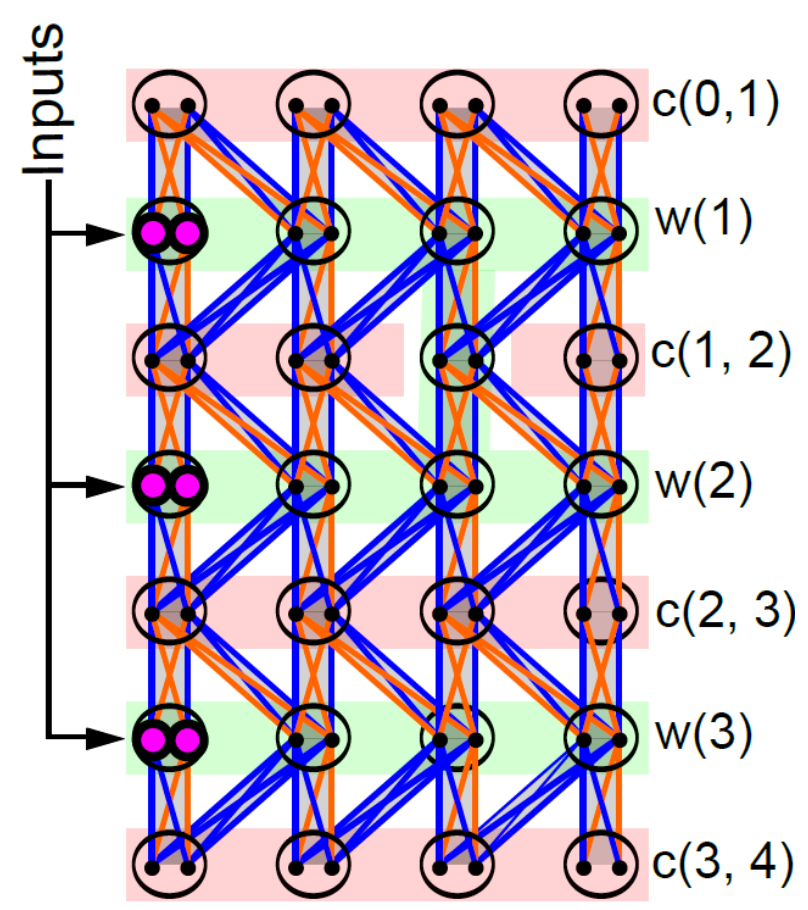

(b)

Figure 4.7: Two MBQC protocols applied on the bilayer square-lattice cluster state. In both cases, information is encoded on the left (in purple nodes) and flows from left to right along (green) wire segments. Wires are separated by lines of sacrificial qumodes (shown in the red segments). These are referred to as control macronodes c $(i, i+1)$ because measurements on them control whether two single- or one two-qumode gate gets applied on the adjacent wire macronodes $\mathrm{w}(i)$ and $\mathrm{w}(i+1)$. Two-qumode gates are represented by a connecting green segment between two adjacent wires. (a) Standard one-way protocol [14,58 applied to the BSL graph after measuring $\hat{q}$ on all $Y$-polarized qumodes (shown faded). Time-ordering of the nodes has been preserved, resulting in an atypical nodal arrangement of an ordinary squarelattice graph. Information propagates at $45^{\circ}$ to the direction of increasing temporal index. Control nodes are measured in the $\hat{q}$-basis to delete them or in a different basis to implement a two-qumode gate. (b) New MBQC protocol taking advantage of the BSL structure. Both layers of the lattice are used simultaneously and quantum information propagates in the direction of increased temporal index, i.e., horizontally on the figure. All edge weights have magnitude $(2 \sqrt{2})^{-1}$. Control and wire macronodes are now at a constant frequency, as shown. See text for further details. 
A more favorable MBQC protocol that eschews all these complications and makes better use of the structure of the BSL CV cluster state is demonstrated in Ref. [5]. The idea is to use both layers of the graph simultaneously and in a way analogous to the conventional (single-layer) square lattice protocol, as shown in Fig. 4.7(b). Interested readers can find more information about this protocol in Ref. [5].

\subsubsection{Other Types of Cluster States}

By delaying every other node in one layer, we have constructed a square-grid lattice cluster state. We can generate this method to delaying every $N$ nodes in one layer. In this case, a state of interleaved squares and $2 \mathrm{~N}$-polygons will be created. For example, in Fig. 4.8 top, I delay every three nodes in one layer, after the BS, a state of interleaved squares and hexagons is created. Similarly, in Fig. 4.8 bottom, I delay every four nodes in one layer, after the BS, a state of squares and octagons is created.

The different types of time delay can be realized by using different types of MZIs. Although square grid lattice is sufficient for universal quantum computing, other rich structures may also be useful in other quantum information research areas such quantum simulating.

\subsubsection{The Self-inverse Property}

I have already pointed out that this proposal is more scalable and experimentally simpler than the proposals in Ref. [38] and Ref. [4]. Another difference is, in this 
BSL cluster state, one macronode only has two modes, instead of having to have four modes in the other two proposals. This not only simplifies the structure, but also preserves stronger entanglement by having larger edge weights between the entangled modes.

So why in the previous proposals we were using four-mode macronodes for squaregrid, instead of simpler ones? Let's start from linear case, then go back to the squaregrid case. For generating a $\mathbf{A}$ with desired structures from a certain $\mathbf{G}$, which can be manipulated directly by engineering the crystals, it's easier if $\mathbf{G}$ is self-inverse, because in this case $\mathbf{A}$ and $\mathbf{G}$ are the same up to permutations, as I have shown in previous Chapters. In order for $\mathbf{A}$ to be self-inverse, it must satisfy [39]:

$$
\left(\mathbf{A}^{2}\right)_{j k}=\sum_{l} \mathbf{A}_{j l} \mathbf{A}_{l k}=\delta_{j k} \mathbf{I}
$$

Note in here we allow entries $\mathbf{A}_{j k}$ in $\mathbf{A}$ to be matrices, i.e., the graph can have matrix-valued weights. This equation can be interpreted geometrically:

(1) All 2-paths that begin and end on the same node have weights that sum to I, and

(2) All 2-paths that link different nodes have weights that cancel out.

A linear graph with real-valued edges can't satisfy Eq. (4.18), because in general there's only one 2-path between different nodes, and it must be zero according to condition (2), then the graph will have zero weights: the multiplication being zero means one of the terms must be zero. But this is not true if the weights are matrices. 
The simplest self-inverse graph must have 2-by-2 matrix-valued weights, as in Eq. (19) in Ref. [39]. One can easily check it satisfies both conditions. The two matrix-valued weights are constructed by using the two orthogonal 2- $D$ vectors.

What about the square-grid case? One can easily check that the graph with the 4-by-4 matrix-valued weights shown in Eq. (22) in Ref. 39] satisfies Eq. 4.18). In Ref. [4, 17], the graphs also have 4-by-4 matrix-valued weights. The four matrixvalued weights are constructed by using the four orthogonal $4-D$ vectors, this choice is very natural because it uses the orthogonality and completeness of the four vectors to fulfill Eq. (4.18). It's harder to design a matrix-valued square grid with matrices of lower dimensions.

However, the BSL has 2-by-2 matrix-valued weights, rather than 4-by-4. As I pointed out, it's not easy to satisfy Eq. 4.18) with 2-by-2 matrices for a square grid structure. Indeed, as shown in Fig. 4.9 left, if we check the macronode in the solid circle, it doesn't satisfy Eq. 4.18)! However, the A for the BSL must be self-inverse, because we start from a self-inverse G (TMSS Hamiltonian), after the BS transform it remains self-inverse. This contradiction can be explained by mode permutation. For example, for a self-inverse matrix $\mathbf{A}$, we have $\mathbf{A}^{2}=\mathbf{I}$. If we permutate the elements in $\mathbf{A}$, then the square of $\mathbf{A}$ will equal to a permutated $\mathbf{I}$, so Eq. (4.18) wil be violated. Indeed, if we look at Fig. 4.9 right, which is topologically same as Fig. 4.9 left up to some modes rearrangements. Now one macronode has six neighbors instead of four, so it is not a square grid in the macronode picture, but this graph satisfies Eq. 4.18). 


\subsubsection{Conclusion of the hybrid cluster states proposal}

The vast majority of the existing literature on continuous-variable (CV) cluster states has treated canonical CV cluster states (i.e., those described in Refs. 14, 18, 58]) as the appropriate target for an MBQC resource state. The body of work by the present authors (and our collaborators) has by now shown definitively that this emphasis on canonical CV cluster states is misplaced [4, 5, 26, 35, 36, 38, 39]. Instead, our schemes - which are all based on bipartite, self-inverse graphs and thus necessarily have a macronode structure [39] - have been demonstrated to have unprecedented scalability [26,36] and to admit novel, flexible, and more efficient quantum-computing schemes within the MBQC paradigm [5]. The work presented here further underscores this point, again emphasizing the importance of bipartite, self-inverse graphs and of the focus on scalable designs from the ground up when working with CV cluster states.

We have proposed an extremely compact and scalable method for producing - from a single OPO and simple interferometer - a CV cluster state of unprecedented size $\left[\left(3 \times 10^{3}\right) \times \infty\right]$ that is universal for quantum computation. The proposal has all the advantages of record-breaking temporal- and frequency-multiplexed schemes $[26,36$ while vastly increasing the size of the lattice by utilizing both types of multiplexing at once. This is the most compact and scalable proposal for CV cluster states to date, and it is implementable today using demonstrated quantum-optical technology. Complementing this proposal, we have also developed a novel scheme for MBQC that 
is tailored for this resource state and that has all the advantages of other schemes based on scalable macronode-based CV cluster states [5].

With the ultimate goal being fault-tolerant quantum computing, future work (beyond performing the experiment proposed here) includes lowering the amount of squeezing required for fault tolerance [61], experimentally demonstrating squeezing at that level, and demonstrating the required error correction [59 61]. 

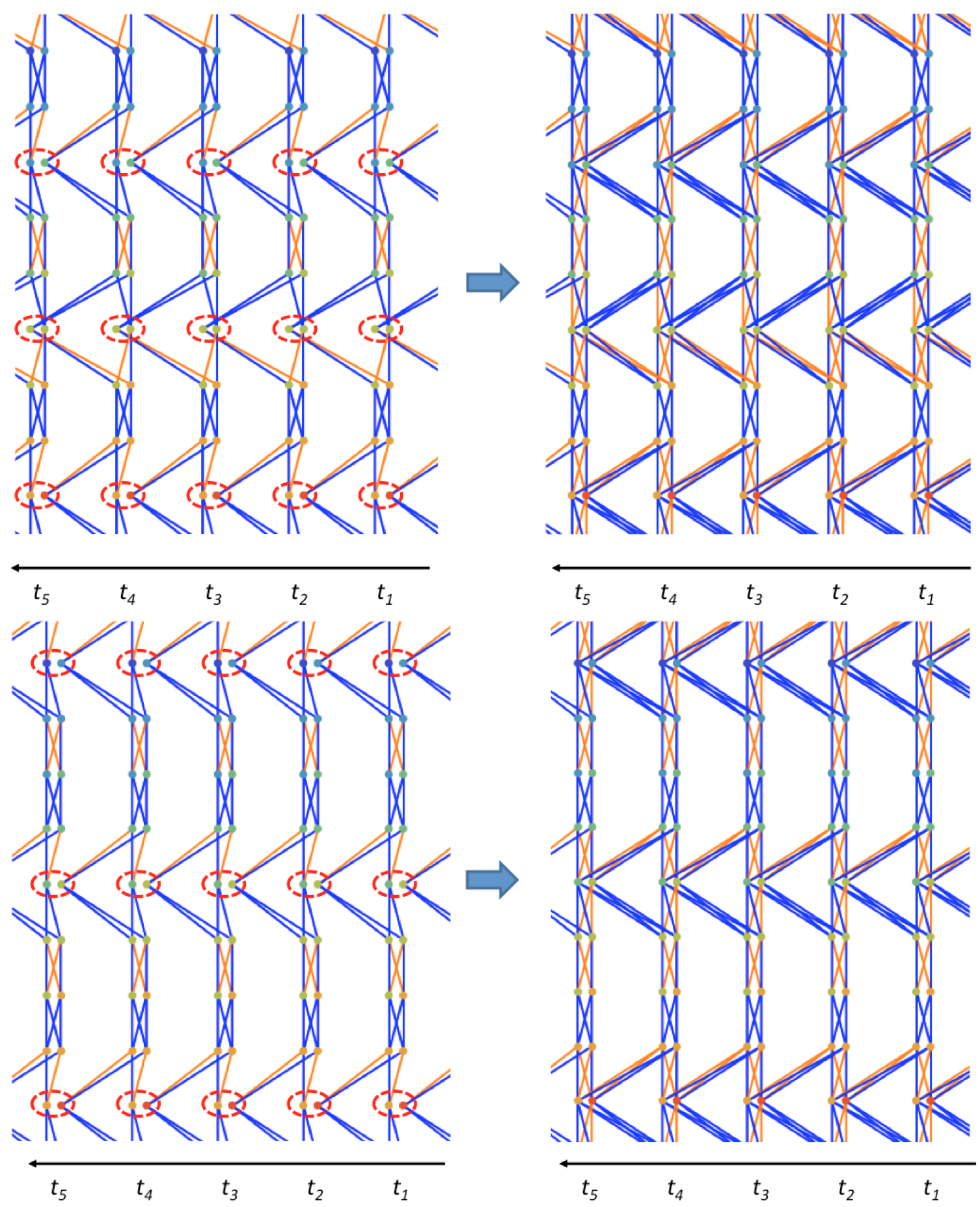

Figure 4.8: Instead of delaying every two nodes in one layer, we can skip an arbitrary number of nodes when applying the time delay. Top: delaying every three nodes in one layer, resulting a state of interleaved squares and hexagons. Bottom: delaying every four nodes in one layer, resulting a state of interleaved squares and octagons. 

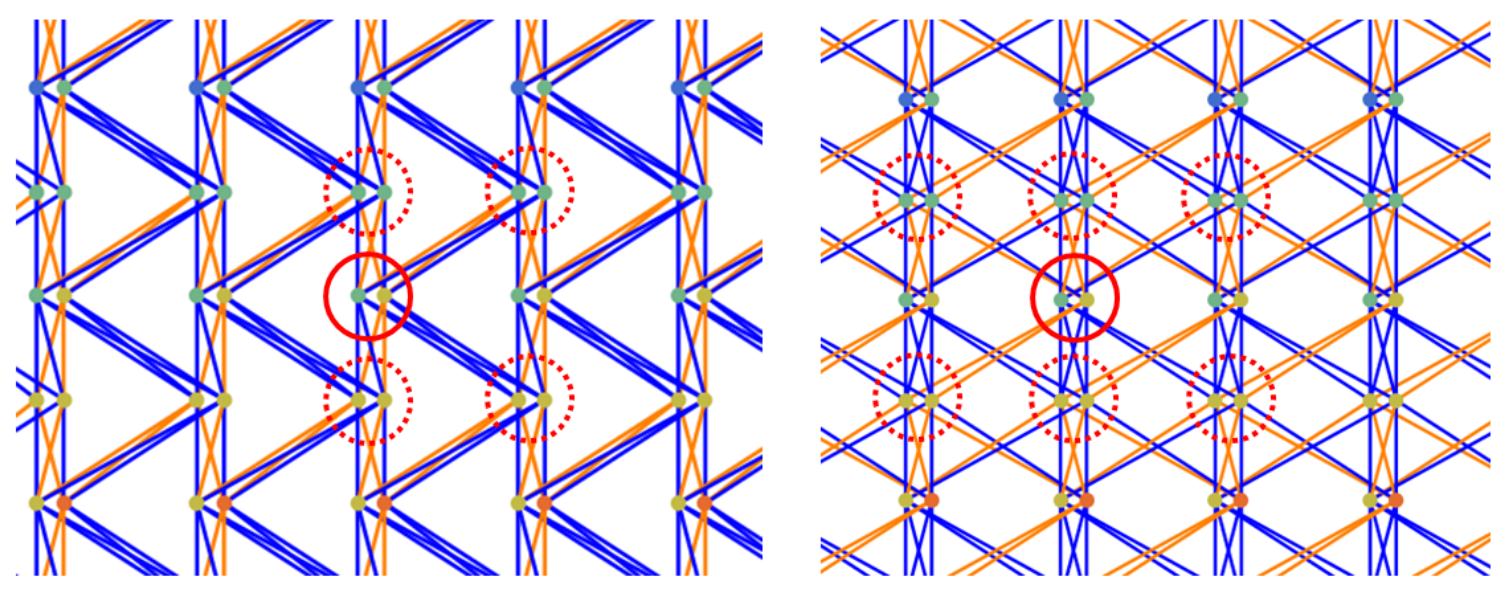

Figure 4.9: Left: the BSL cluster state. Every macronode (in solid circle) has four neighbors (in dashed circles). Right: the modes in BSL cluster state are rearranged by reversing the time delay and grouping the two modes at the same time as a macronode. Every macronode (in solid circle) has six neighbors (in dashed circles). 


\section{Chapter 5}

\section{Conclusion}

In this thesis, I have introduced measurement-based quantum computers implemented by quantum optics, and focused on two experimental proposals for generating large scale lattice cluster states that can be used for universal quantum computing and other quantum information process.

One of the biggest advantages of optical qumodes (continuous variables) over qubits is the scalability. The world records for the largest entangled cluster states were achieved by using a single optical parametric oscillator (OPO) [26, 36]. The most convenient way to describe such a scalable system is to use graphical approaches. I have introduced the $\mathbf{A}$ graph, the $\mathcal{H}$ graph and the $\mathbf{Z}$ graph, and have presented how to derive one graph from the other.

The most important process in generating cluster states on QOFC is the parametric down-convertion (PDC), which is a second order nonlinear process. I have reviewed the PDC and other nonlinear optical processes, and demonstrated the limitations of qumodes scalability in both theory and experiment [53]. 
I have presented a scalable experimental proposal for generating giant cluster states with hypercubic lattice structures [4]. I have shown that with only $D$ OPOs, a $D$-dimensional hypercubic cluster state can be generated in the frequency domain. This proposal significantly simplified the requirements for creating square-grid cluster states in the previous proposals [38], and the high dimensional lattice structure opens possibilities for other quantum information research topics.

I have demonstrated a recent proposal for generating a time-frequency hybrid square-grid cluster state, with a single OPO [5]. This method is the most scalable method ever proposed: the total number of entangled modes in the generated state is $10^{3} \times \infty$ in theory. Besides the scalability and simplicity of the proposal, we have also developed a more efficient method for performing universal quantum computing by making use of all qumodes in the square-grid cluster states. I have also shown with a simple modification, cluster states with different structures can be generated by the same setup.

Our ultimate goal is to realize fault-tolerant quantum computing. Although the finite squeezing in the continuous variable implementation introduces noise to $\mathrm{CV}$ cluster states, by using the Gottesman-Kitaev-Preskill (GKP) encoding [59], quantum error correction can be fulfilled if the initial squeezing level of the cluster states is above $20.5 \mathrm{~dB}$ [61]. The future works in this area include lowering the amount of squeezing, experimentally demonstrating squeezing at required level, and implement the error correction encoding [60]. 


\section{Bibliography}

[1] P. W. Shor. Algorithms for quantum computation: discrete logarithms and factoring. In S. Goldwasser, editor, Proceedings, $35^{\text {th }}$ Annual Symposium on Foundations of Computer Science, pages 124-134, Santa Fe, NM, 1994. IEEE Press, Los Alamitos, CA.

[2] Richard P. Feynman. Simulating physics with computers. Int. J. Theor. Phys., 21:467-488, 1982.

[3] H. J. Briegel and R. Raussendorf. Persistent entanglement in arrays of interacting particles. Phys. Rev. Lett., 86:910, 2001.

[4] Pei Wang, Moran Chen, Nicolas C. Menicucci, and Olivier Pfister. Weaving quantum optical frequency combs into hypercubic cluster states. Phys. Rev. A, 90:032325, 2014.

[5] Niranjan Sridhar Moran Chen Olivier Pfister Nicolas C. Menicucci Rafael N. Alexander, Pei Wang. One-way quantum computing with arbitrarily large time-frequency continuous-variable cluster states from a single optical parametric oscillator. arXiv:1509.00484, 2015. 
[6] T. D. Ladd, F. Jelezko, R. Laflamme, Y. Nakamura, C. Monroe, and J. L. O’Brien. Quantum computers. Nature (London), 464:45, 2010.

[7] A. R. Rivest, A. Shamir and L. Adleman. A method for obtaining digital signatures and public-key cryptosystems. Communications of the ACM, 21:120, 1978.

[8] D. Deutsch and R. Jozsa. Rapid solution of problems by quantum computation. Proc. Roy. Soc. Lond. A, 439(1907):553-558, 1992.

[9] Michael A. Nielsen and Isaac L. Chuang. Quantum computation and quantum information. Cambridge University Press, Cambridge, U.K., 2000.

[10] R. Raussendorf and H. J. Briegel. A one-way quantum computer. Phys. Rev. Lett., 86:5188, 2001.

[11] Bruce E Kane. A silicon-based nuclear spin quantum computer. nature, 393(6681):133-137, 1998.

[12] Juan I Cirac and Peter Zoller. Quantum computations with cold trapped ions. Physical review letters, 74(20):4091, 1995.

[13] E Knill, R Laflamme, and G J Milburn. A scheme for efficient quantum computation with linear optics. Nature (London), 409:46-52, January 2001. 
[14] N. C. Menicucci, P. van Loock, M. Gu, C. Weedbrook, T. C. Ralph, and M. A. Nielsen. Universal quantum computation with continuous-variable cluster states. Phys. Rev. Lett., 97:110501, 2006.

[15] M. Hein, J. Eisert, and H. J. Briegel. Multiparty entanglement in graph states. Phys. Rev. A, 69(6):062311, 2004.

[16] A. Einstein, B. Podolsky, and N. Rosen. Can quantum-mechanical description of physical reality be considered complete? Phys. Rev., 47:777, 1935.

[17] Nicolas C. Menicucci, Steven T. Flammia, and Peter van Loock. Graphical calculus for Gaussian pure states with applications to continuous-variable cluster states. Phys. Rev. A, 83:042335, 2011.

[18] Jing Zhang and Samuel L. Braunstein. Continuous-variable Gaussian analog of cluster states. Phys. Rev. A, 73(3):032318, 2006.

[19] P. Hyllus and J. Eisert. Optimal entanglement witnesses for continuous-variable systems. New J. Phys., 8(4):51, 2006.

[20] Matthew Pysher, Yoshichika Miwa, Reihaneh Shahrokhshahi, Russell Bloomer, and Olivier Pfister. Parallel generation of quadripartite cluster entanglement in the optical frequency comb. Phys. Rev. Lett., 107(3):030505, Jul 2011.

[21] B. E. A. Saleh and M. C. Teich. Fundamentals of Photonics. Wiley and Sons, New York, NY, 1991. 
[22] J. M. Manley and H. E. Rowe. Some general properties of nonlinear elements part i: General energy relations. Proceedings of the IRE, page 904, 1956.

[23] VG Dmitriev, GG Gurzadyan, and DN Nikogosyan. Handbook of nonlinear optical crystals, (1999). Springer Series in Optical Sciences, Springer, Berlin, ISBN 3540653945.

[24] K. Fradkin, A. Arie, A. Skliar, and G. Rosenman. Tunable midinfrared source by difference frequency generation in bulk periodically poled $\mathrm{KTiOPO}_{4}$. Appl. Phys. Lett., 74(7):914-916, 1999.

[25] S. Emanueli and A. Arie. Temperature-dependent dispersion equations for $\mathrm{KTiOPO}_{4}$ and $\mathrm{KTiOAsO}_{4}$. Appl. Opt., 42:6661, 2003.

[26] Moran Chen, Nicolas C. Menicucci, and Olivier Pfister. Experimental realization of multipartite entanglement of 60 modes of a quantum optical frequency comb. Phys. Rev. Lett., 112:120505, Mar 2014.

[27] H. Fang, A. Picard, and P. Juncar. A heterodyne refractometer for air index of refraction and air density measurements. Rev. Sci. Instrum., 73(4):1934-1938, 2002.

[28] T.Y. Fan, C.E. Huang, B.Q. Hu, R.C. Eckardt, Y.X. Fan, R.L. Byer, and R.S. Feigelson. Second harmonic generation and accurate index of refraction measurements in flux-grown ktiopo 4 . Appl. Opt., 26:2390, 1987. 
[29] GM Gale, M Cavallari, TJ Driscoll, and F Hache. Sub-20-fs tunable pulses in the visible from an 82-mhz optical parametric oscillator. Optics letters, 20(14):15621564, 1995.

[30] ES Wachman, DC Edelstein, and CL Tang. Continuous-wave mode-locked and dispersion-compensated femtosecond optical parametric oscillator. Optics letters, 15(2):136-138, 1990.

[31] Nicolai Matuschek, Franz X Kartner, and Ursula Keller. Analytical design of double-chirped mirrors with custom-tailored dispersion characteristics. Quantum Electronics, IEEE Journal of, 35(2):129-137, 1999.

[32] J Hebling, R Szipöcs, EJ Mayer, and J Kuhl. Chirped-mirror dispersioncompensated femtosecond optical parametric oscillator. Optics letters, 20(8):919921, 1995.

[33] Nick Leindecker, Alireza Marandi, Robert L Byer, and Konstantin L Vodopyanov. Broadband degenerate opo for mid-infrared frequency comb generation. Optics express, 19(7):6296-6302, 2011.

[34] Nick Leindecker, Alireza Marandi, Robert L Byer, Konstantin L Vodopyanov, Jie Jiang, Ingmar Hartl, Martin Fermann, and Peter G Schunemann. Octavespanning ultrafast opo with 2.6-6.1 $\mu \mathrm{m}$ instantaneous bandwidth pumped by femtosecond tm-fiber laser. Optics express, 20(7):7046-7053, 2012. 
[35] Nicolas C. Menicucci. Temporal-mode continuous-variable cluster states using linear optics. Phys. Rev. A, 83(6):062314, Jun 2011.

[36] Shota Yokoyama, Ryuji Ukai, Seiji C. Armstrong, Chanond Sornphiphatphong, Toshiyuki Kaji, Shigenari Suzuki, Jun-ichi Yoshikawa, Hidehiro Yonezawa, Nicolas C. Menicucci, and Akira Furusawa. Ultra-large-scale continuous-variable cluster states multiplexed in the time domain. Nat. Photon., 7:982, 2013.

[37] Markus Greiner, Olaf Mandel, Tilman Esslinger, Theodor W. Hänsch, and Immanuel Bloch. Quantum phase transition from a superfluid to a mott insulator in a gas of ultracold atoms. Nature (London), 415:39, 2002.

[38] Nicolas C. Menicucci, Steven T. Flammia, and Olivier Pfister. One-way quantum computing in the optical frequency comb. Phys. Rev. Lett., 101:130501, 2008.

[39] S. T. Flammia, N. C. Menicucci, and O. Pfister. The optical frequency comb as a one-way quantum computer. J. Phys. B,, 42:114009, 2009.

[40] H. Zaidi, N. C. Menicucci, S. T. Flammia, R. Bloomer, M. Pysher, and O. Pfister. Entangling the optical frequency comb: simultaneous generation of multiple $2 \times 2$ and $2 \times 3$ continuous-variable cluster states in a single optical parametric oscillator. Laser Phys., 18:659, 2008. Revised version at http://arxiv.org/pdf/0710.4980v3.

[41] N. C. Menicucci, S. T. Flammia, H. Zaidi, and O. Pfister. Ultracompact generation of continuous-variable cluster states. Phys. Rev. A, 76:010302(R), 2007. 
[42] R. Simon, E. C. G. Sudarshan, and N. Mukunda. Gaussian pure states in quantum mechanics and the symplectic group. Phys. Rev. A, 37:3028-3038, Apr 1988.

[43] Dragomir Ž Doković. Hadamard matrices of order 764 exist. Combinatorica, 28(4):487-489, 2008.

[44] Todd K. Moon. Error correction coding. Wiley, 2005.

[45] Marek Zukowski, Anton Zeilinger, and Michael A. Horne. Realizable higherdimensional two-particle entanglements via multiport beam splitters. Phys. Rev. A, 55:2564-2579, Apr 1997.

[46] Y. Ben-Aryeh. Multiport beam-splitter controlled transformations with postselection of superposition single-photon states. Opt. Comm., 283(14):2863-2865, 2010.

[47] Nicolas C. Menicucci. Continuous-variable cluster states in the optical frequency comb are robust to local errors in the Hamiltonian-connection graph used to make them. in preparation, 2013.

[48] Daoxin Dai, Zhi Wang, Jared F. Bauters, M.-C. Tien, Martijn J. R. Heck, Daniel J. Blumenthal, and John E Bowers. Low-loss $s i_{3} n_{4}$ arrayed-waveguide grating (de)multiplexer using nano-core optical waveguides. Opt. Expr., 19(15):14130-14136, Jul 2011. 
[49] M. Shirasaki. Large angular dispersion by a virtually imaged phased array and its application to a wavelength demultiplexer. Opt. Lett., 21:366-368, 1996.

[50] Scott A. Diddams, Leo Hollberg, and Vela Mbele. Molecular fingerprinting with the resolved modes of a femtosecond laser frequency comb. Nature (London), 445:627, 2007.

[51] Tommaso F. Demarie, Trond Linjordet, Nicolas C. Menicucci, and Gavin K. Brennen. Detecting topological entanglement entropy in a lattice of quantum harmonic oscillators. arXiv:1305.0409 [quant-ph], May 2013.

[52] E Dennis, A Kitaev, A Landahl, and J Preskill. Topological quantum memory. J. Math. Phys., 43:4452-4505, October 2001.

[53] Pei Wang, Wenjiang Fan, and Olivier Pfister. Engineering large-scale entanglement in the quantum optical frequency comb: influence of the quasiphasematching bandwidth and of dispersion. arXiv:1403.6631 [physics.optics], 2014.

[54] O. Glöckl, U. L. Andersen, S. Lorenz, Ch. Silberhorn, N. Korolkova, and G. Leuchs. Sub-shot-noise phase quadrature measurement of intense light beams. Opt. Lett., 29(16):1936-1938, Aug 2004.

[55] E. H. Huntington, G. N. Milford, C. Robilliard, T. C. Ralph, O. Glöckl, U. L. Andersen, S. Lorenz, and G. Leuchs. Demonstration of the spatial separation of the entangled quantum sidebands of an optical field. Phys. Rev. A, 71:041802, Apr 2005. 
[56] S. Reynaud, C. Fabre, and E. Giacobino. Quantum fluctuations in a two-mode parametric oscillator. J. Opt. Soc. Am. B, 4:1520, 1987.

[57] D. F. Walls and G. J. Milburn. Quantum Optics. Springer, Berlin, 1994.

[58] Mile Gu, Christian Weedbrook, Nicolas C. Menicucci, Timothy C. Ralph, and Peter van Loock. Quantum computing with continuous-variable clusters. Phys. Rev. A, 79:062318, 2009.

[59] Daniel Gottesman, Alexei Kitaev, and John Preskill. Encoding a qubit in an oscillator. Phys. Rev. A, 64:012310, 2001.

[60] H. M. Vasconcelos, L. Sanz, and S. Glancy. All-optical generation of states for "Encoding a qubit in an oscillator". Opt. Lett., 35(19):3261-3263, Oct 2010.

[61] Nicolas C. Menicucci. Fault-tolerant measurement-based quantum computing with continuous-variable cluster states. Phys. Rev. Lett., 112:120504, Mar 2014. 